\title{
Catalytic Asymmetric Total Synthesis of Hypocrolide A
}

Chuang Qiao, ${ }^{+,+}$Wen Zhang, ${ }^{\ddagger}$ Jing-Chun Han ${ }^{\ddagger}$ and Chuang-Chuang Li ${ }^{*+,+\neq}$

${ }^{\dagger}$ School of Chemical Biology and Biotechnology, Peking University Shenzhen Graduate School, Shenzhen 518055, China

${ }^{*}$ Department of Chemistry, South University of Science and Technology of China, Shenzhen 518055, China

E-mail: ccli@sustc.edu.cn

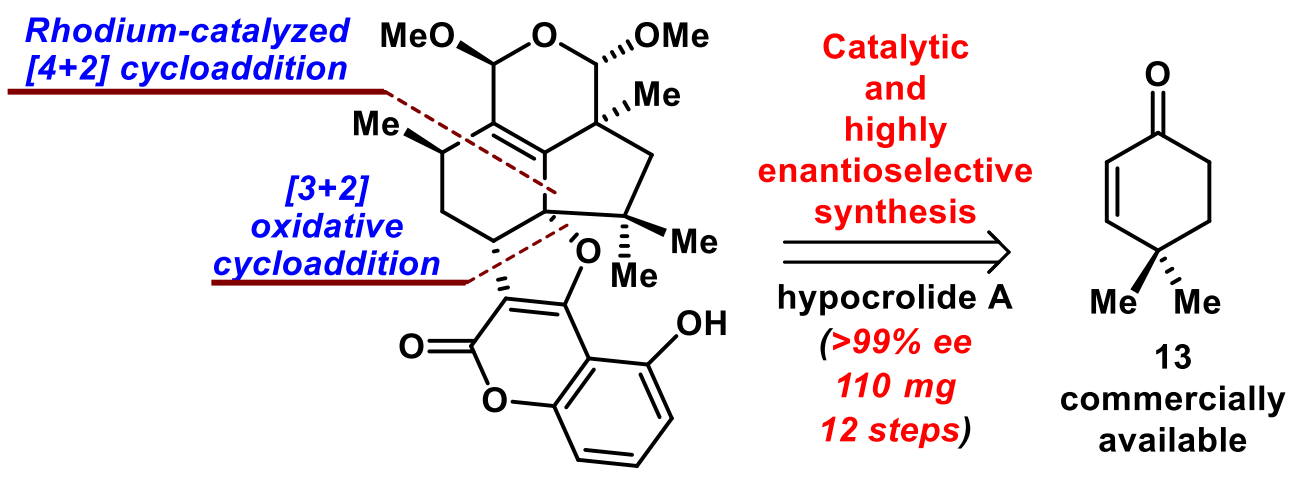




\section{Supplementary Materials}

\section{Table of Contents}

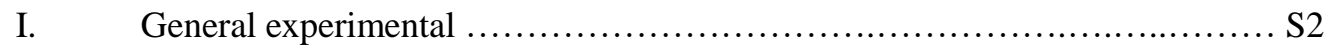

II. Experimental Procedures............................................ S3 31

III. X-ray crystal structures................................................ S32-S33

IV. $\quad{ }^{1} \mathrm{H}$ and ${ }^{13} \mathrm{C}$ NMR Spectra............................................ S34-S77 


\section{General Information}

Unless otherwise mentioned, all reactions were carried out under a nitrogen atmosphere under anhydrous conditions and all reagents were purchased from commercial suppliers without further purification. Solvent purification was conducted according to Purification of Laboratory Chemicals (Peerrin, D. D.; Armarego, W. L. and Perrins, D. R., Pergamon Press: Oxford, 1980). Yields refer to chromatographically and spectroscopically ( ${ }^{1} \mathrm{H}$ NMR) homogeneous materials, unless otherwise stated. Reactions were monitored by Thin Layer Chromatography on plates (GF254) supplied by Yantai Chemicals (China) using UV light as visualizing agent, an ethanolic solution of phosphomolybdic acid, or basic aqueous potassium permanganate (KMnO4), and heat as developing agents.. If not specially mentioned, flash column chromatography uses silica gel (200-300 mesh) supplied by Tsingtao Haiyang Chemicals (China), Preparative thin layer chromatography (PTLC) separations were carried out $0.50 \mathrm{~mm}$ Yantai (China) silica gel plates. NMR spectra were recorded on Bruker AV500, Bruker ARX400 and Bruker AV300 instruments. and calibrated using residual undeuterated solvent as an internal reference $\left(\mathrm{CHCl}_{3}, \delta 7.26 \mathrm{ppm}{ }^{1} \mathrm{H}\right.$ NMR, $\delta 77.00{ }^{13} \mathrm{C}$ NMR). The following abbreviations were used to explain the multiplicities: $\mathrm{s}=$ singlet, $\mathrm{d}=$ doublet, $\mathrm{t}=$ triplet, $\mathrm{q}=$ quartet, $\mathrm{b}=$ broad, $\mathrm{m}=$ multiplet .

High-resolution mass spectra (HRMS) were recorded on a Bruker Apex IV FTMS mass spectrometer using ESI (electrospray ionization). Infrared spectra were recorded on a Shimadzu IR Prestige 21, using thin films of the sample on $\mathrm{KBr}$ plates. Optical rotations were measured with a Rudolph autopol I automatic polarimeter using $10 \mathrm{~cm}$ glass cells with a sodium $589 \mathrm{~nm}$ filter. 


\section{Experimental Procedures}

\section{Synthesis of 12}<smiles>CC1(C)C=CC(=O)CC1</smiles>

13
[14-2TFA]

(10 mol\%)

$\mathrm{H}_{2} \mathrm{O}_{2}$ (30 wt\%)

dioxane (0.25 M)

$40^{\circ} \mathrm{C}, 48 \mathrm{~h}$

$90 \%$

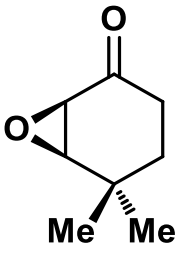

12

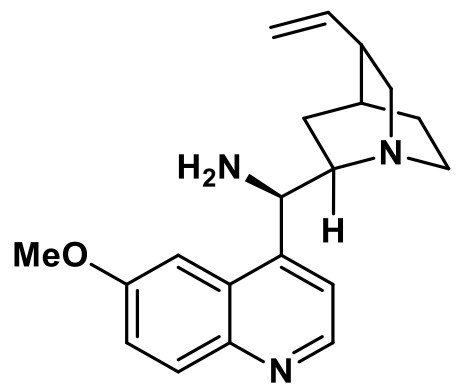

14

Added 9-amino(9-deoxy)epiquinidine $14(5.2 \mathrm{~g}, 16.1 \mathrm{mmol}, 10 \mathrm{~mol} \%)$ to a solution of trifluoroacetic acid (TFA; $2.4 \mathrm{~mL}, 32.2 \mathrm{mmol}, 20 \mathrm{~mol} \%$ ) in dioxane (650 mL) to form 9- $\mathrm{NH}_{2}$-epiQ-2TFA. Then, cyclic enone $\mathbf{1 3}$ (20.0 g, $161 \mathrm{mmol}, 1.0$ equiv) was added, and 20 minutes later, aqueous hydrogen peroxide (30 wt $\% ; 27.5 \mathrm{~mL}, 242 \mathrm{mmol}, 1.5$ equiv). After $48 \mathrm{~h}$ of stirring at $40{ }^{\circ} \mathrm{C}$, the reaction mixture was extracted with $\mathrm{Et}_{2} \mathrm{O}(3 \times 300 \mathrm{~mL})$. The combined organic phases were dried over anhydrous sodium sulfate, filtered and concentrated under reduced pressure. The crude product was purified by silica gel column chromatography (hexane/ethyl acetate $=50 / 1)$ to give $\mathbf{1 2}(20.4 \mathrm{~g}, 90 \%$ yield $)$ as a colorless oil .

$\mathbf{R}_{f}=0.4$ (hexane/ethyl acetate $=50 / 1$ );

$[\boldsymbol{\alpha}]_{\mathbf{D}}^{\mathbf{2 5}}=+98.0\left(\mathrm{c}=1.0\right.$ in $\left.\mathrm{CHCl}_{3}\right) ;$

IR (film) $\lambda_{\max } 2960,2883,1671,1554,1110,1059,1018,860$;

${ }^{1} \mathbf{H}$ NMR (400 MHz, $\left.\mathbf{C D C l}_{3}\right) \delta 3.21(\mathrm{~d}, J=4.0 \mathrm{~Hz}, 1 \mathrm{H}), 3.16(\mathrm{dd}, J=4.0,1.1 \mathrm{~Hz}, 1 \mathrm{H}), 2.35-$ $2.42(\mathrm{~m}, 1 \mathrm{H}), 2.17-2.22(\mathrm{~m}, 1 \mathrm{H}), 1.86-1.92(\mathrm{~m}, 1 \mathrm{H}), 1.33-1.35(\mathrm{~m}, 1 \mathrm{H}), 1.20(\mathrm{~s}, 3 \mathrm{H}), 1.05(\mathrm{~s}$ $3 \mathrm{H})$;

${ }^{13}$ C NMR (100 MHz, $\left.\mathbf{C D C l}_{3}\right) \delta$ 206.0, 64.1, 55.9, 33.1, 30.7, 29.7, 27.4, 22.8;

HRMS (ESI) calcd for $\mathrm{C}_{8} \mathrm{H}_{13} \mathrm{O}_{2}{ }^{+}[\mathrm{M}+\mathrm{H}]^{+}$Exact Mass: 141.0910; found: 141.0911.

Synthesis of 12a<smiles>CC1(C)CCC(=O)[C@H]2O[C@H]21</smiles>

12

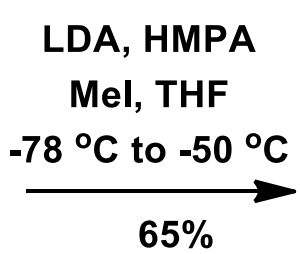

$65 \%$

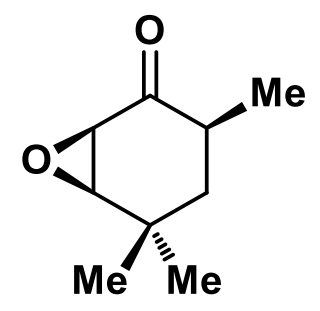

$12 a$ 
A solution of (1S,6S)-5,5-dimethyl-7-oxabicyclo[4.1.0]heptan-2-one (1.0 g, $7.1 \mathrm{mmol}, 1.0$ equiv) in tetrahydrofuran $(30 \mathrm{~mL})$ was added to a solution of LDA ( $2 \mathrm{M}$ in tetrahydrofuran, $4.3 \mathrm{~mL}, 8.6$ mmol, 1.2 equiv) and anhydrous HMPA ( $4.5 \mathrm{~mL}, 25.6 \mathrm{mmol}, 3.6$ equiv) at $-78{ }^{\circ} \mathrm{C}$. After stirring for $0.5 \mathrm{~h}$, the reaction mixture was raised to $-50{ }^{\circ} \mathrm{C}$ and stirred for another $2.0 \mathrm{~h}$. Iodomethane (0.66 mL, $10.7 \mathrm{mmol}, 1.5$ equiv) was added dropwise to the flask, and then the mixture was stirred for another $3.0 \mathrm{~h}$ at the same temperature. A saturated aqueous solution of $\mathrm{NH}_{4} \mathrm{Cl}(30 \mathrm{~mL})$ was added and the mixture was extracted with ethyl acetate $(3 \times 30 \mathrm{~mL})$. The combined organic phases were dried over anhydrous sodium sulfate, filtered and concentrated under reduced pressure. The crude product was purified by silica gel column chromatography (hexane/ethyl acetate $=50 / 1$ ) to give 12a $(0.72 \mathrm{~g}, 65 \%$ yield $)$ as a colorless oil.

Note: We have got 10.0 gram of compound 12 a from $20.0 \mathrm{~g}$ of compound 12 in one pot, $46 \%$ yield.

$\mathbf{R}_{f}=0.5$ (hexane/ethyl acetate $\left.=50 / 1\right) ;$

$[\boldsymbol{\alpha}]_{\mathbf{D}}^{22}=+100.5\left(\mathrm{c}=1.0\right.$ in $\left.\mathrm{CHCl}_{3}\right)$

IR (film) $\lambda_{\max } 2959,2926,2872,1703,1464,1364,1220,1048,1026,881,839,773$;

${ }^{1}$ H NMR $\left(\mathbf{5 0 0} \mathbf{M H z}, \mathbf{C D C l}_{\mathbf{3}}\right) \delta 3.21(\mathrm{~d}, J=3.8 \mathrm{~Hz}, 1 \mathrm{H}), 3.15(\mathrm{~d}, J=3.6 \mathrm{~Hz}, 1 \mathrm{H}), 2.24-2.09(\mathrm{~m}$, 1H), $1.63(\mathrm{t}, J=13.0 \mathrm{~Hz}, 1 \mathrm{H}), 1.36(\mathrm{dd}, J=13.6,6.4 \mathrm{~Hz}, 1 \mathrm{H}), 1.19(\mathrm{~s}, 3 \mathrm{H}), 1.10(\mathrm{~d}, J=7.0 \mathrm{~Hz}$, $3 \mathrm{H}), 1.08(\mathrm{~s}, 3 \mathrm{H})$;

${ }^{13}$ C NMR (125 MHz, $\left.\mathbf{C D C l}_{3}\right) \delta 207.4,63.5,55.4,38.1,37.5,31.1,27.8,22.7,16.2$;

HRMS (ESI) calcd for $\mathrm{C}_{9} \mathrm{H}_{15} \mathrm{O}_{2}{ }^{+}[\mathrm{M}+\mathrm{H}]^{+}$Exact Mass: 155.1067; found: 155.1068 .

\section{Synthesis of $12 b$}

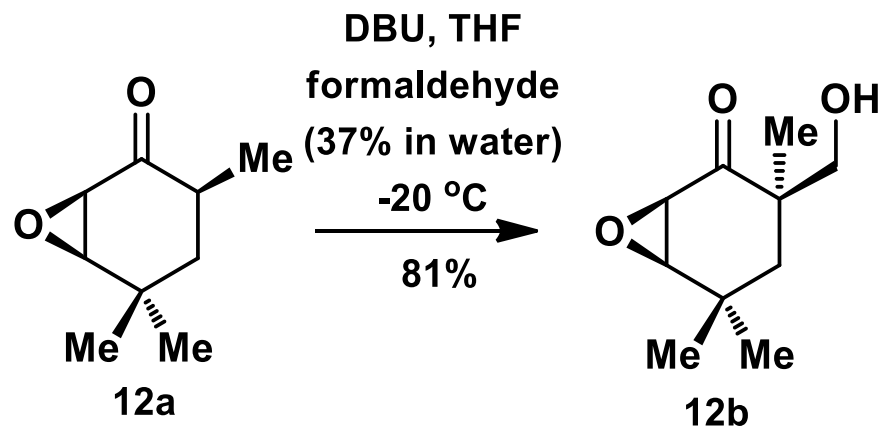

DBU (46.6 ml, 311.6 mmol, 2.0 equiv) and formaldehyde (622 mmol, $37 \%$ solution in $\mathrm{H}_{2} \mathrm{O}, 4.0$ equiv) were dissolved in THF (300 mL) at $-20{ }^{\circ} \mathrm{C}$. Then 12a $(24.0 \mathrm{~g}, 155.8 \mathrm{mmol}, 1.0$ equiv) was 
added dropwise to the mixture, and stirred for $3.0 \mathrm{~h}$ at the same temperature. A saturated aqueous solution of $\mathrm{NH}_{4} \mathrm{Cl}(100 \mathrm{~mL})$ was added and the mixture was extracted with EtOAc $(3 \times 200 \mathrm{~mL})$. The combined organic phases were dried over anhydrous sodium sulfate, filtered and concentrated under reduced pressure. The crude product was purified by silica gel column chromatography (hexane/ethyl acetate $=5 / 1)$ to give $\mathbf{1 2 b}(23.2 \mathrm{~g}, 81 \%$ yield $)$ as a light yellow oil.

$\mathbf{R}_{f}=0.4$ (hexane/ethyl acetate $=3 / 1$ );

$[\boldsymbol{\alpha}]_{\mathbf{D}}^{25}=+125.0\left(\mathrm{c}=1.0\right.$ in $\left.\mathrm{CHCl}_{3}\right)$;

IR (film) $\lambda_{\max } 2975,2960,2893,1716,1261,1220,773,668$;

${ }^{1}$ H NMR (400 MHz, $\left.\mathbf{C D C l}_{3}\right) \delta 3.53(\mathrm{dd}, J=10.6,4.1 \mathrm{~Hz}, 1 \mathrm{H}), 3.44(\mathrm{dd}, J=10.5,7.5 \mathrm{~Hz}, 1 \mathrm{H}), 3.35$ (d, $J=4.3 \mathrm{~Hz}, 1 \mathrm{H}), 3.29$ (dd, $J=4.3,1.5 \mathrm{~Hz}, 1 \mathrm{H}), 2.05-1.98(\mathrm{~m}, 1 \mathrm{H}), 1.97(\mathrm{~d}, J=14.1 \mathrm{~Hz}, 1 \mathrm{H}), 1.21$ (s, 3H), $1.18(\mathrm{~d}, J=14.1 \mathrm{~Hz}, 1 \mathrm{H}), 1.15(\mathrm{~s}, 3 \mathrm{H}), 1.08(\mathrm{~s}, 3 \mathrm{H})$;

${ }^{13}$ C NMR (10 MHz, $\mathbf{C D C l}_{3}$ ) $\delta$ 211.0, 70.2, 65.8, 56.4, 46.6, 41.1, 31.9, 28.4, 24.9, 23.4;

HRMS (ESI) calcd for $\mathrm{C}_{10} \mathrm{H}_{17} \mathrm{O}_{3}^{+}[\mathrm{M}+\mathrm{H}]^{+}$Exact Mass: 185.1172; found: 185.1171.

\section{Synthesis of 11a}

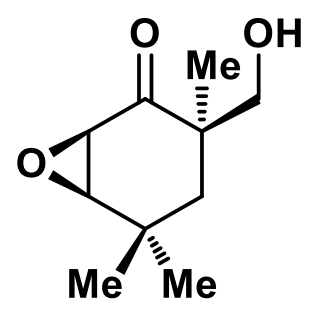

$12 b$

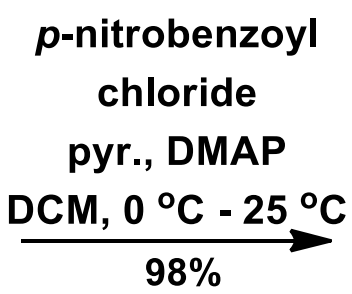

To a solution of compound $\mathbf{1 2 b}\left(50 \mathrm{mg}, 0.27 \mathrm{mmol}, 1.0\right.$ equiv) in $\mathrm{CH}_{2} \mathrm{Cl}_{2}(30 \mathrm{~mL})$ at $0{ }^{\circ} \mathrm{C}$ was added pyridine ( $0.03 \mathrm{~mL}, 0.41 \mathrm{mmol}, 1.5$ equiv), DMAP $(10 \mathrm{mg})$, and $p$-nitrobenzoyl chloride (66 $\mathrm{mg}, 0.54 \mathrm{mmol}, 2.0$ equiv). The reaction mixture was allowed to warm to $25^{\circ} \mathrm{C}$ and stirred for $2 \mathrm{~h}$. The mixture was concentrated under reduced pressure and purified by silica gel column chromatography (hexane/ethyl acetate $=20 / 1)$ to give $11 \mathrm{a}(97 \mathrm{mg}, 71 \%$ yield) as a white solid.

$\mathbf{R}_{f}=0.7$ (hexane/ethyl acetate $\left.=20 / 1\right)$

$[\boldsymbol{\alpha}]_{\mathrm{D}}^{27}=+30.1\left(\mathrm{c}=1.0\right.$ in $\left.\mathrm{CHCl}_{3}\right) ;$

IR (film) $\lambda_{\max } ; 2961,2930,1728,1708,1607,1528,1468,1348,1271,1119,1103,1015,989,873$, 
$844,719,646$

${ }^{1}$ H NMR (500 MHz, CDCl $) \delta 8.36-8.09(\mathrm{~m}, 4 \mathrm{H}), 4.32(\mathrm{~d}, J=10.6 \mathrm{~Hz}, 1 \mathrm{H}), 4.22(\mathrm{~d}, J=10.7$ Hz, 1H), 3.43 (d, $J=4.3 \mathrm{~Hz}, 1 \mathrm{H}), 3.32(\mathrm{dd}, J=4.3,1.2 \mathrm{~Hz}, 1 \mathrm{H}), 2.15(\mathrm{~d}, J=13.9 \mathrm{~Hz}, 1 \mathrm{H}), 1.30$ (dd, $J=13.9,1.3 \mathrm{~Hz}, 1 \mathrm{H}), 1.23$ (s, 3H), 1.22 (s, 3H), 1.11 (s, 3H);

${ }^{13}$ C NMR (125MHz, $\left.\mathbf{C D C l}_{3}\right) \delta$ 209.07, 164.35, 150.59, 135.16, 130.91, 123.57, 71.22, 65.65, $56.49,44.72,41.25,32.08,28.50,24.82,23.79$;

HRMS (ESI) calcd for $\mathrm{C}_{17} \mathrm{H}_{19} \mathrm{NNaO}_{6}{ }^{+}[\mathrm{M}+\mathrm{Na}]^{+}$Exact Mass: 356.1105 ; found: 356.1107 .

\section{Synthesis of 11}
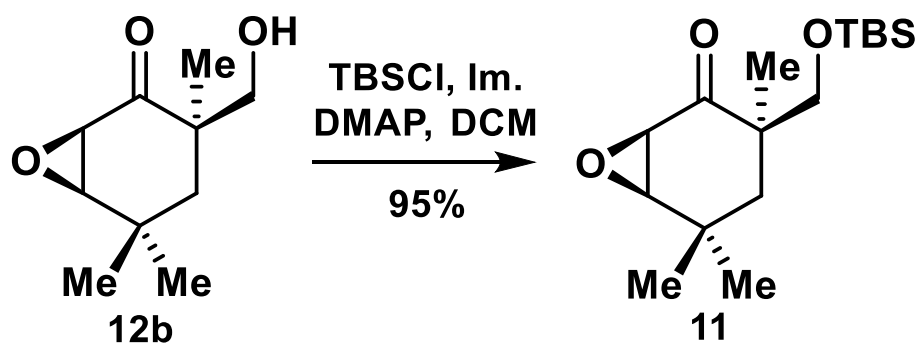

To a solution of $(1 S, 3 R, 6 S)$-3-(hydroxymethyl)-3,5,5-trimethyl-7-oxabicyclo[4.1.0]heptan-2-one (15.0 g, $81.4 \mathrm{mmol}, 1.0$ equiv), imidazole (5.6 g, $81.4 \mathrm{mmol}, 1.0$ equiv) and catalytic amount of DMAP (500 mg, $4.1 \mathrm{mmol}, 0.05$ equiv) in $\mathrm{CH}_{2} \mathrm{Cl}_{2}(200 \mathrm{~mL})$ was added TBSCl (14.8 g, 97.7 mmol, 1.2 equiv), the mixture was stirred at $25{ }^{\circ} \mathrm{C}$ for $12 \mathrm{~h}$. A saturated aqueous solution of $\mathrm{NH}_{4} \mathrm{Cl}(100 \mathrm{~mL})$ was added and the mixture was extracted with $\mathrm{CH}_{2} \mathrm{Cl}_{2}(3 \times 200 \mathrm{~mL})$. The combined organic phases were dried over anhydrous sodium sulfate, filtered and concentrated under reduced pressure. The crude product was purified by silica gel column chromatography (hexane/ethyl acetate $=100 / 1)$ to give $\mathbf{1 1}(23.0 \mathrm{~g}, 95 \%$ yield $)$ as a light yellow oil.

$\mathbf{R}_{f}=0.6$ (hexane/ethyl acetate $\left.=50 / 1\right) ;$

$[\boldsymbol{\alpha}]_{\mathrm{D}}^{27}=+84.5\left(\mathrm{c}=1.0\right.$ in $\left.\mathrm{CHCl}_{3}\right) ;$

IR (film) $\lambda_{\max } 2958,2931,2858,1733,1699,1464,1456,1260,1108,935,836$;

${ }^{1}$ H NMR $\left(400 \mathrm{MHz}, \mathbf{C D C l}_{\mathbf{3}}\right) \delta 3.50(\mathrm{~d}, J=9.2 \mathrm{~Hz}, 1 \mathrm{H}), 3.44(\mathrm{~d}, J=9.2 \mathrm{~Hz}, 1 \mathrm{H}), 3.28(\mathrm{~d}, J=4.3$ $\mathrm{Hz}, 1 \mathrm{H}), 3.19-3.21(\mathrm{~m}, 1 \mathrm{H}), 2.04(\mathrm{~d}, J=14.0 \mathrm{~Hz}, 1 \mathrm{H}), 1.16(\mathrm{~s}, 3 \mathrm{H}), 1.10(\mathrm{dd}, J=14.0,1.6 \mathrm{~Hz}$, 1H), $1.06(\mathrm{~s}, 3 \mathrm{H}), 1.02(\mathrm{~s}, 3 \mathrm{H}), 0.86(\mathrm{~s}, 9 \mathrm{H}),-0.01(\mathrm{~s}, 6 \mathrm{H})$;

${ }^{13}$ C NMR (100 MHz, $\left.\mathbf{C D C l}_{3}\right) \delta 210.5,70.2,65.6,56.5,47.2,41.5,32.0,28.5,25.7,24.7,23.3$, $18.2,-5.6,-5.6$

HRMS (ESI) calcd for $\mathrm{C}_{16} \mathrm{H}_{30} \mathrm{O}_{3} \mathrm{NaSi}^{+}[\mathrm{M}+\mathrm{Na}]^{+}$Exact Mass: 321.1856; found: 321.1856 . 


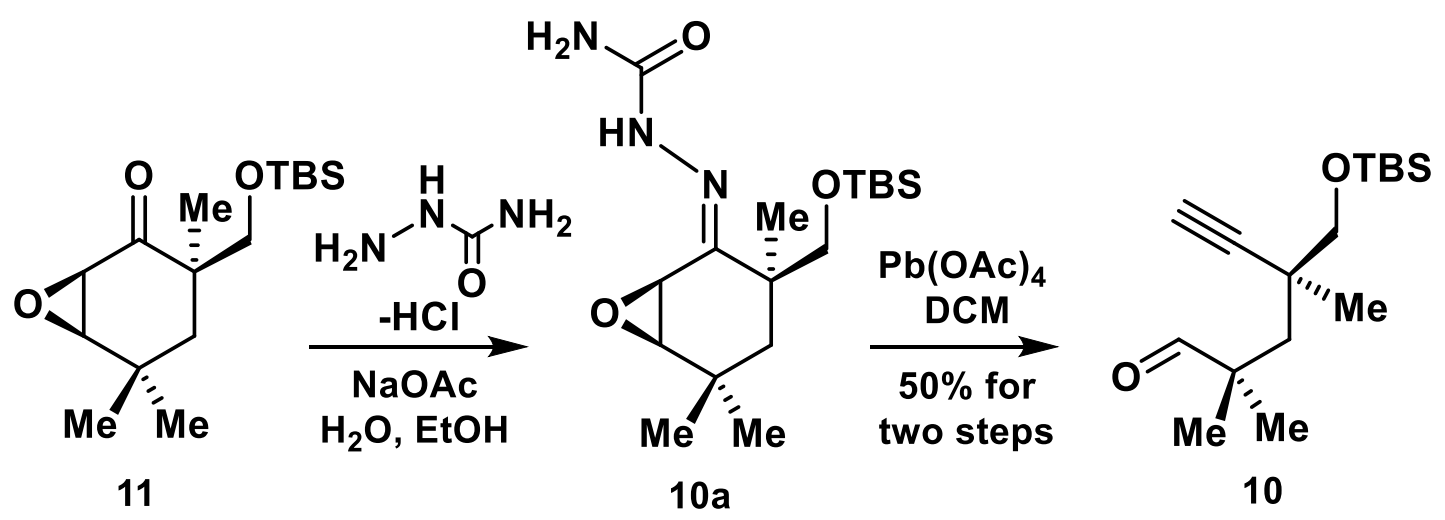

To a stirred solution of semicarbazide hydrochloride ( $26.5 \mathrm{~g}, 351.8 \mathrm{mmol}, 5.0$ equiv) and sodium acetate $(11.5 \mathrm{~g}, 140.8 \mathrm{mmol}, 2.0$ equiv) in water $(200 \mathrm{~mL})$ was added $\mathbf{1 1}(21.0 \mathrm{~g}, 70.4 \mathrm{mmol}, 1.0$ equiv) at $25{ }^{\circ} \mathrm{C}$. Sufficient $95 \%$ ethanol $(500 \mathrm{~mL})$ was added to bring about a homogenous solution. After stirring at $25{ }^{\circ} \mathrm{C}$ for about $12 \mathrm{~h}$, the reaction mixture was extracted with dichloromethane $(3 \times 300 \mathrm{~mL})$. The combined organic phases were dried over anhydrous sodium sulfate, filtered and concentrated under reduced pressure. Without separation, the crude product 10a was dissolved in anhydrous $\mathrm{CH}_{2} \mathrm{Cl}_{2}(500 \mathrm{~mL})$, then lead tetraacetate $(95 \%, 33.0 \mathrm{~g}, 70.4 \mathrm{mmol}$, 1.0 equiv) was added portionwise at $0{ }^{\circ} \mathrm{C}$. After stirring for $1 \mathrm{~h}$ at $0{ }^{\circ} \mathrm{C}$, enough $\mathrm{H}_{2} \mathrm{O}(300 \mathrm{~mL})$ was added to quench the reaction. The mixture was filtered through a celite pad and extracted with $\mathrm{CH}_{2} \mathrm{Cl}_{2}(3 \times 300 \mathrm{~mL})$. The organic phases were combined and neutralized with a saturated aqueous solution of $\mathrm{NaHCO}_{3}(100 \mathrm{~mL})$, dried over anhydrous sodium sulfate, filtered and concentrated under reduced pressure. The crude product was purified by silica gel column chromatography (hexane/ethyl acetate $=50 / 1)$ to give $\mathbf{1 0}(10.0 \mathrm{~g}, 50 \%$ yield $)$ as a colorless oil.

$\mathbf{R}_{f}=0.7$ (hexane/ethyl acetate $\left.=50 / 1\right) ;$

$[\boldsymbol{\alpha}]_{\mathbf{D}}^{26}=+13.3\left(\mathrm{c}=1.0\right.$ in $\left.\mathrm{CHCl}_{3}\right) ;$

IR (film) $\lambda_{\max } 2956,2929,2857.59,1728,1472,1363,1251,1098,1006,837,776,669,636$;

${ }^{1}$ H NMR (400 MHz, $\left.\mathbf{C D C l}_{3}\right) \delta 9.56(\mathrm{~s}, 1 \mathrm{H}), 3.49(\mathrm{~d}, J=9.5 \mathrm{~Hz}, 1 \mathrm{H}), 3.38(\mathrm{~d}, J=9.5 \mathrm{~Hz}, 1 \mathrm{H})$, $2.11(\mathrm{~s}, 1 \mathrm{H}), 1.95(\mathrm{~d}, J=14.6 \mathrm{~Hz}, 1 \mathrm{H}), 1.70(\mathrm{~d}, J=14.6 \mathrm{~Hz}, 1 \mathrm{H}), 1.18$ (s, 3H), 1.16 (s, 3H), 1.14 (s, 3H), $0.89(\mathrm{~d}, J=2.9 \mathrm{~Hz}, 9 \mathrm{H}), 0.05(\mathrm{~s}, 6 \mathrm{H})$.

${ }^{13}$ C NMR (100 MHz, $\left.\mathbf{C D C l}_{3}\right) \delta$ 206.4, 88.6, 71.8, 70.4, 46.2, 44.0, 35.9, 29.7, 25.9, 25.8, 23.5, 23.1, 18.3;

HRMS (ESI) calcd for $\mathrm{C}_{16} \mathrm{H}_{30} \mathrm{O}_{2} \mathrm{NaSi}^{+}[\mathrm{M}+\mathrm{Na}]^{+}$Exact Mass: 305.1907; found: 305.1908 . 


\section{Synthesis of 9}

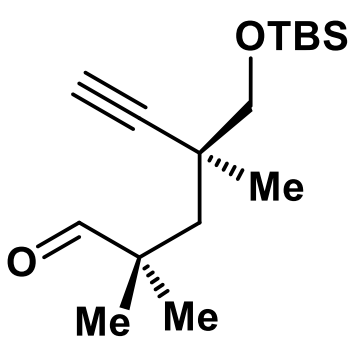

10

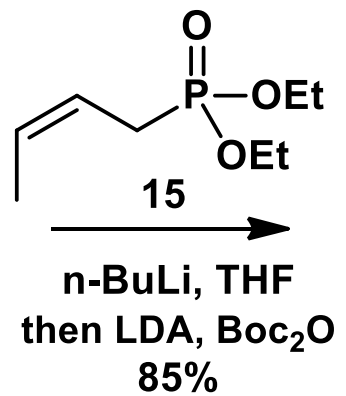

$85 \%$

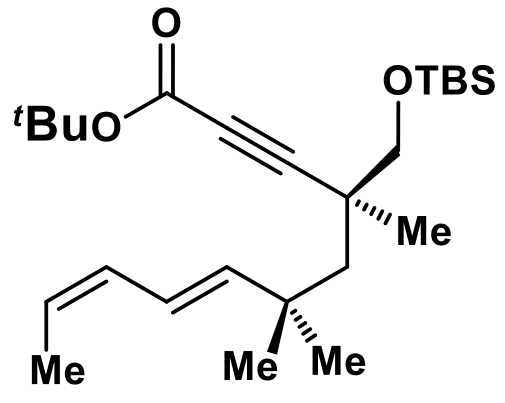

9

To a suspension of diethyl (Z)-but-2-en-1-ylphosphonate 15 (Binns, M. R.; Haynes, R. K.; Katsifis, A. G.; Schober, P. A.; Vonwiller, S. C. J. Am. Chem. Soc. 1988, 110, 5411.) (1.8 g, 9.6 mmol, 3.0 equiv) in anhydrous $\mathrm{THF}(250 \mathrm{~mL})$ at $-78^{\circ} \mathrm{C}$ was added dropwise $\mathrm{n}$-butyllithium $(2.5 \mathrm{M}$ in hexane, $3.8 \mathrm{~mL}, 9.6 \mathrm{mmol}, 3.0$ equiv). The reaction mixture was stirred for $0.5 \mathrm{~h}$ at the same temperature, and then the reaction device was transferred into an ice-water bath and the reaction mixture was stirred for another $0.5 \mathrm{~h}$. Aldehyde $\mathbf{1 0}(900 \mathrm{mg}, 3.2 \mathrm{mmol}, 1.0$ equiv) was dissolved in anhydrous THF (30 mL) and injected dropwise into the reaction system. The reaction was gradually warmed to $25{ }^{\circ} \mathrm{C}$ for $0.5 \mathrm{~h}$. Recooling the mixture to $-78^{\circ} \mathrm{C}$, then it was added another solution of LDA (1 $\mathrm{M}$ in tetrahydrofuran, $4.8 \mathrm{~mL}, 4.8 \mathrm{mmol}, 1.5$ equiv) and stirring for $0.5 \mathrm{~h}$. Then the reaction device was transferred into an ice-water bath and the reaction mixture was stirred for another $0.5 \mathrm{~h}$. $\mathrm{Boc}_{2} \mathrm{O}(1.5 \mathrm{~mL}, 6.4 \mathrm{mmol}, 2.0$ equiv) was added to the flask, then the mixture was allowed to stirred for another $0.5 \mathrm{~h}$. A saturated aqueous solution of $\mathrm{NH}_{4} \mathrm{Cl}(30 \mathrm{~mL})$ was added and the mixture was extracted with ethyl acetate $(3 \times 30 \mathrm{~mL})$. The combined organic phases were dried over anhydrous sodium sulfate, filtered and concentrated under reduced pressure. The crude product was purified by silica gel column chromatography (hexane/ethyl acetate $=20 / 1$ ) to give 9 $(1.1 \mathrm{~g}, 85 \%$ yield) as a colorless oil.

$\mathbf{R}_{f}=0.75$ (hexane/ethyl acetate $=20 / 1$ );

$[\boldsymbol{\alpha}]_{\mathrm{D}}^{26}=+5.8\left(\mathrm{c}=1.0\right.$ in $\left.\mathrm{CHCl}_{3}\right)$

IR (film) $\lambda_{\max } 2957,2926,2855,2361,2332,1811,1757,1709,1458,1373,1260,1119,1078$, 1020, 800;

${ }^{1} \mathbf{H}$ NMR $\left(\mathbf{5 0 0}\right.$ MHz, $\left.\mathbf{C D C l}_{3}\right) \delta 6.26(\mathrm{ddd}, J=15.5,10.8,0.9 \mathrm{~Hz}, 1 \mathrm{H}), 6.06-5.93(\mathrm{~m}, 1 \mathrm{H}), 5.79$ $(\mathrm{d}, J=15.5 \mathrm{~Hz}, 1 \mathrm{H}), 5.37(\mathrm{dq}, \mathrm{J}=10.8,7.1 \mathrm{~Hz}, 1 \mathrm{H}), 3.49(\mathrm{~d}, J=9.4 \mathrm{~Hz}, 1 \mathrm{H}), 3.44(\mathrm{~d}, J=9.4 \mathrm{~Hz}$, 
1H) $1.77-1.69(\mathrm{~m}, 4 \mathrm{H}), 1.54(\mathrm{~d}, J=14.3 \mathrm{~Hz}, 1 \mathrm{H}), 1.47(\mathrm{~s}, 9 \mathrm{H}), 1.20(\mathrm{~s}, 3 \mathrm{H}), 1.18(\mathrm{~s}, 6 \mathrm{H}), 1.17(\mathrm{~s}$, $6 \mathrm{H}), 0.90(\mathrm{~s}, 9 \mathrm{H}), 0.04(\mathrm{~s}, 3 \mathrm{H}), 0.04(\mathrm{~s}, 3 \mathrm{H})$;

${ }^{13}$ C NMR (125 MHz, $\mathbf{C D C l}_{3}$ ) $\delta 153.1,144.3,130.0,124.0,120.9,91.6,82.5,76.8,70.2,48.7$,

$36.9,36.9,29.3,29.1,28.0,25.9,25.0,18.3,13.4,-5.4,-5.5$;

HRMS (ESI) calcd for $\mathrm{C}_{25} \mathrm{H}_{44} \mathrm{O}_{3} \mathrm{NaSi}^{+}[\mathrm{M}+\mathrm{Na}]^{+}$Exact Mass: 443.2952; found: 443.2953

\section{Synthesis of 9a}

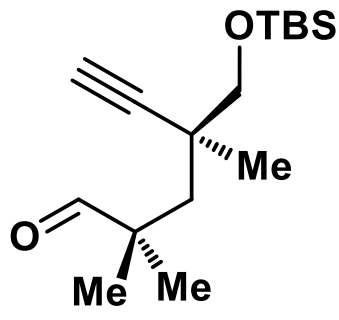

10

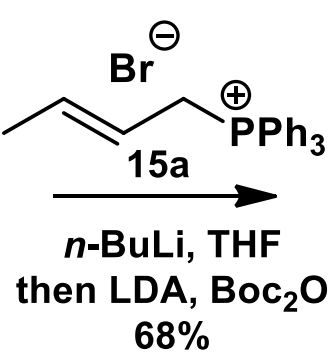

$68 \%$

To a suspension of (E)-but-2-en-1-yltriphenylphosphonium bromide 15a (Geiserler, O.; Podlech, J. Tetrahedron 2012, 68, 7280.) (28.0 g, $70.7 \mathrm{mmol}, 3.0$ equiv) in anhydrous THF (250 mL) at $-78{ }^{\circ} \mathrm{C}$ was added dropwise n-butyllithium (2.5 $\mathrm{M}$ in hexane, $28.3 \mathrm{~mL}, 70.7 \mathrm{mmol}, 3.0$ equiv). The reaction mixture was stirred for $0.5 \mathrm{~h}$ at the same temperature, and then the reaction device was transferred into an ice-water bath and the reaction mixture was stirred for another $0.5 \mathrm{~h}$. Aldehyde $\mathbf{1 0}$ (6.6 g, $23.3 \mathrm{mmol}, 1.0$ equiv) was dissolved in anhydrous THF (40 mL) and injected dropwise into the reaction system. The reaction was gradually warmed to room temperature for $0.5 \mathrm{~h}$. Recooling the mixture to $-78^{\circ} \mathrm{C}$, then it was added another solution of LDA ( $1 \mathrm{M}$ in tetrahydrofuran, $120.9 \mathrm{~mL}, 120.9 \mathrm{mmol}, 1.5$ equiv) and stirring for $0.5 \mathrm{~h}$. Then the reaction device was transferred into an ice-water bath and the reaction mixture was stirred for another $0.5 \mathrm{~h}$. $\mathrm{Boc}_{2} \mathrm{O}(14.5 \mathrm{~g}, 161.2 \mathrm{mmol}, 2.0$ equiv) was added to the flask, then the mixture was allowed to stirred for another $0.5 \mathrm{~h}$. A saturated aqueous solution of $\mathrm{NH}_{4} \mathrm{Cl}(100 \mathrm{~mL})$ was added and the mixture was extracted with ethyl acetate $(3 \times 200 \mathrm{~mL})$. The combined organic phases were dried over anhydrous sodium sulfate, filtered and concentrated under reduced pressure. The crude product was purified by silica gel column chromatography (hexane/ethyl acetate $=20 / 1$ ) to give $9 \mathbf{a}$ (6.8 g, 68\% yield) as a colorless oil.

$\mathbf{R}_{f}=0.7$ (hexane/ethyl acetate $\left.=20 / 1\right) ;$ 
$[\boldsymbol{\alpha}]_{\mathbf{D}}^{22}=+15.9\left(\mathrm{c}=0.50\right.$ in $\left.\mathrm{CHCl}_{3}\right)$

IR (film) $\lambda_{\max }$ 2956, 2929, 2857.59, 1728, 1472, 1363, 1251, 1098, 1006, 837, 776, 669, 636;

${ }^{1} \mathbf{H}$ NMR (400 MHz, $\left.\mathbf{C D C l}_{3}\right) \delta 6.60-6.43(\mathrm{~m}, 1 \mathrm{H}), 5.78(\mathrm{t}, J=11.9 \mathrm{~Hz}, 1 \mathrm{H}), 5.58(\mathrm{dq}, J=13.6,6.7$ $\mathrm{Hz}, 1 \mathrm{H}), 5.28(\mathrm{~d}, J=12.1 \mathrm{~Hz}, 1 \mathrm{H}), 3.48(\mathrm{q}, J=9.3 \mathrm{~Hz}, 2 \mathrm{H}), 1.79-1.67(\mathrm{dd}, J=7.8,6.4 \mathrm{~Hz}, 4 \mathrm{H}), 1.59$ (d, $J=14.3 \mathrm{~Hz}, 1 \mathrm{H}), 1.47$ (s, 9H), 1.30 (s, 3H), 1.28 (s, 3H), 1.23 (s, 3H), 0.89 (s, 9H), 0.05 (s, 3H), $0.05(\mathrm{~s}, 3 \mathrm{H})$

${ }^{13} \mathbf{C}$ NMR (100 MHz, $\left.\mathbf{C D C l}_{3}\right) \delta 153.2,138.5,130.2,128.1,127.1,92.0,82.5,70.6,49.3,37.6,37.0$, $31.3,30.9,28.0,25.8,24.7,18.4,18.3,-5.5,-5.5$;

HRMS (ESI) calcd for $\mathrm{C}_{25} \mathrm{H}_{44} \mathrm{O}_{3} \mathrm{NaSi}^{+}[\mathrm{M}+\mathrm{Na}]^{+}$Exact Mass: 443.2952; found: 443.2952 .

Synthesis of 8 and 16 by rhodium-catalyzed intramolecular [4+2] cycloaddition<smiles>C/C=C\C=C\C(C)(C)C[C@@](C)(C#CC(=O)OCC)CO[R5](=O)[O-]</smiles>

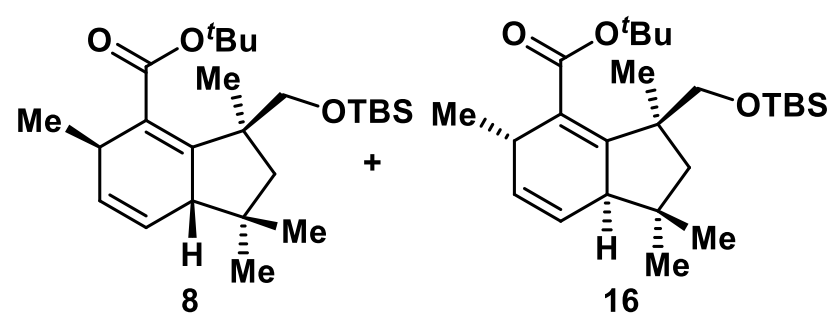

Compound 9 (3.0 g, $7.1 \mathrm{mmol}, 1.0$ equiv) and $[\mathrm{Rh}(\mathrm{COD}) \mathrm{Cl}]_{2}(35 \mathrm{mg}, 0.07 \mathrm{mmol}, 0.01$ equiv) were dissolved in 2,2,2-trifluoroethanol (TFE, $100 \mathrm{~mL}$ ) under nitrogen atmosphere and the solution was stirred for $2 \mathrm{~h}$ at $25{ }^{\circ} \mathrm{C}$. Then the mixture was directly concentrated under reduced pressure. The crude product was purified by silica gel column chromatography (hexane/ethyl acetate $=20 / 1)$ to give $\mathbf{8}(2.28 \mathrm{~g}, 76.0 \%)$ as a light yellow solid and $\mathbf{1 6}(0.50 \mathrm{~g}, 15.0 \%$ yield $)$ as a light yellow oil.

Note: The internal Z,E-diene 9a also readily underwent the desired rhodium-catalyzed [4+2] cycloaddition under the same conditions, to afford $\mathbf{8}$ and $\mathbf{1 6}$ in $80 \%$ yield. However, $\mathbf{8}$ and $\mathbf{1 6}$ was in a ratio of 1.1:1.

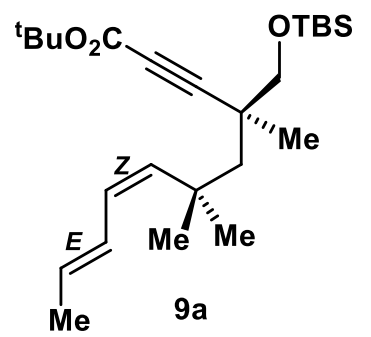

$9 a$

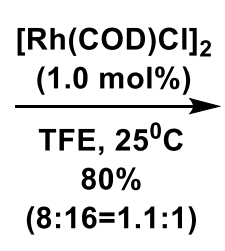

$(8: 16=1 \cdot 1: 1)$

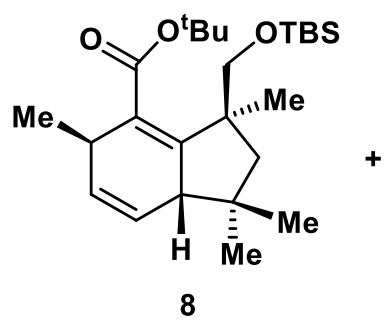

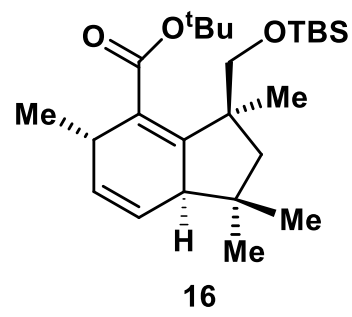




\section{Compound 8:}

$\mathbf{R}_{f}=0.8$ (hexane/ethyl acetate $\left.=20 / 1\right) ;$

$[\boldsymbol{\alpha}]_{\mathbf{D}}^{27}=+12.9\left(\mathrm{c}=1.0\right.$ in $\left.\mathrm{CHCl}_{3}\right)$

IR (film) $\lambda_{\max }$ 2957, 2928, 2859, 2361, 1717, 1462, 1391, 1368, 1252, 11589, 1094, 1069, 837, 804, 775;

${ }^{1}$ H NMR (500 MHz, CDCl $) \delta 5.70-5.74(\mathrm{~m}, 1 \mathrm{H}), 5.54-5.56(\mathrm{~m}, 1 \mathrm{H}), 3.93(\mathrm{~d}, J=8.7 \mathrm{~Hz}, 1 \mathrm{H})$, $3.44(\mathrm{~d}, J=8.7 \mathrm{~Hz}, 1 \mathrm{H}), 3.26-3.10(\mathrm{~m}, 1 \mathrm{H}), 2.75-2.58(\mathrm{~m}, 1 \mathrm{H}), 2.10(\mathrm{~d}, J=12.8 \mathrm{~Hz}, 1 \mathrm{H}), 1.50$ (s, 9H), $1.23(\mathrm{~d}, J=12.8 \mathrm{~Hz}, 1 \mathrm{H}), 1.13(\mathrm{~s}, 3 \mathrm{H}), 1.08(\mathrm{~s}, 3 \mathrm{H}), 1.00(\mathrm{~d}, J=7.0 \mathrm{~Hz}, 3 \mathrm{H}), 0.90-0.85$ (m, 9H), $0.71(\mathrm{~s}, 3 \mathrm{H}), 0.03(\mathrm{~s}, 6 \mathrm{H})$;

${ }^{13}$ C NMR (125 MHz, $\left.\mathbf{C D C l}_{3}\right) \delta$ 169.6, 149.8, 130.79, 129.2, 122.8, 80.4, 71.3, 52.9, 51.9, 46.0, $38.8,34.9,28.1,27.5,25.9,24.2,23.1,21.0,18.1,-5.3,-5.4$;

HRMS (ESI) calcd for $\mathrm{C}_{25} \mathrm{H}_{44} \mathrm{O}_{3} \mathrm{NaSi}^{+}[\mathrm{M}+\mathrm{Na}]^{+}$Exact Mass: 443.2952; found: 443.2953 .

\section{Compound 16:}

$\mathbf{R}_{f}=0.7$ (hexane/ethyl acetate $=20 / 1$;

$[\boldsymbol{\alpha}]_{\mathbf{D}}^{26}=+10.2\left(\mathrm{c}=1.0\right.$ in $\left.\mathrm{CHCl}_{3}\right)$

IR (film) $\lambda_{\max }$ 2957, 2928, 2857, 2359, 1713, 1634, 1464, 1368, 1252, 1165, 1088, 1057, 837, 776; ${ }^{1}$ H NMR (500 MHz, $\left.\mathbf{C D C l}_{3}\right) \delta 5.70-5.74(\mathrm{~m}, 1 \mathrm{H}), 5.61-5.54(\mathrm{~m}, 1 \mathrm{H}), 3.72(\mathrm{~d}, J=9.1 \mathrm{~Hz}, 1 \mathrm{H})$, $3.38(\mathrm{~d}, J=9.1 \mathrm{~Hz}, 1 \mathrm{H}), 3.21-3.10(\mathrm{~m}, 1 \mathrm{H}), 2.77-2.79(\mathrm{~m}, 2.5 \mathrm{~Hz}, 1 \mathrm{H}), 2.04(\mathrm{~d}, J=13.5 \mathrm{~Hz}$, 1H), $1.51(\mathrm{~s}, 9 \mathrm{H}), 1.32(\mathrm{~s}, 3 \mathrm{H}), 1.23(\mathrm{~d}, J=13.5 \mathrm{~Hz}, 1 \mathrm{H}), 1.08(\mathrm{~s}, 3 \mathrm{H}), 1.02(\mathrm{~d}, J=7.0 \mathrm{~Hz}, 3 \mathrm{H})$, $0.88(\mathrm{~s}, 9 \mathrm{H}), 0.67$ (s, 3H), $0.01(\mathrm{~s}, 6 \mathrm{H})$.

${ }^{13}$ C NMR (125 MHz, $\left.\mathbf{C D C l}_{3}\right) \delta 169.9,148.6,130.9,128.8,122.5,80.6,67.5,51.0,50.9,46.2$, $39.0,34.9,28.1,28.0,27.0,25.9,23.7,20.6,18.2,-5.3,-5.4$;

HRMS (ESI) calcd for $\mathrm{C}_{25} \mathrm{H}_{44} \mathrm{O}_{3} \mathrm{NaSi}^{+}[\mathrm{M}+\mathrm{Na}]^{+}$Exact Mass: 443.2952; found: 443.2953 .

\section{Synthesis of 8aa}

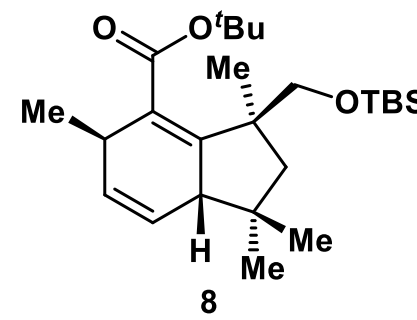

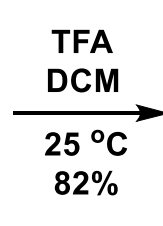

S11 
To a solution of compound $8\left(80 \mathrm{mg}, 0.19 \mathrm{mmol}, 1.0\right.$ equiv) in dichloromethane $(15 \mathrm{~mL})$ at $0{ }^{\circ} \mathrm{C}$ was added TFA $(3 \mathrm{~mL})$. The solution was stirred for $0.5 \mathrm{~h}$ at the same temperature then rise to 25 ${ }^{\circ} \mathrm{C}$. Keep stirring until TLC showed that the starting material disappeared. The mixture was neutralized with a saturated aqueous solution of $\mathrm{NaHCO}_{3}(30 \mathrm{~mL})$, extracted with dichloromethane $(3 \times 30 \mathrm{~mL})$. The combined organic phases were dried over anhydrous sodium sulfate, filtered and concentrated under reduced pressure. The crude product was purified by silica gel column chromatography (hexane/ethyl acetate $=5 / 1)$ to give $\mathbf{8 a a}(36 \mathrm{mg}, 82 \%$ yield) as a light yellow oil.

$\mathbf{R}_{f}=0.5$ (hexane/ethyl acetate $=5 / 1$ );

${ }^{1}$ H NMR (500 MHz, $\left.\mathbf{C D C l}_{3}\right) \delta 5.80-5.77(\mathrm{~m}, 1 \mathrm{H}), 5.57-5.54(\mathrm{~m}, 1 \mathrm{H}), 3.53(\mathrm{dd}, J=23.6,10.1$ $\mathrm{Hz}, 2 \mathrm{H}), 3.38-3.35(\mathrm{~m}, 1 \mathrm{H}), 2.77-2.75(\mathrm{~m}, 1 \mathrm{H}), 1.42(\mathrm{~s}, 2 \mathrm{H}), 1.36(\mathrm{~s}, 3 \mathrm{H}), 1.25(\mathrm{~s}, 1 \mathrm{H}), 1.09$ (s, $3 \mathrm{H}), 1.06(\mathrm{~d}, J=7.0 \mathrm{~Hz}, 3 \mathrm{H}), 0.72(\mathrm{~s}, 3 \mathrm{H})$.

${ }^{13}$ C NMR (125 MHz, $\left.\mathbf{C D C l}_{3}\right) \delta$ 131.0, 121.4, 70.7, 53.1, 51.2, 45.5, 38.4, 35.1, 27.4, 24.1, 22.3, 21.2

HRMS (ESI) calcd for $\mathrm{C}_{15} \mathrm{H}_{21} \mathrm{O}_{2}[\mathrm{M}+\mathrm{H}]^{+}$Exact Mass: 233.1536; found: 233.1536.

\section{Synthesis of 8a}
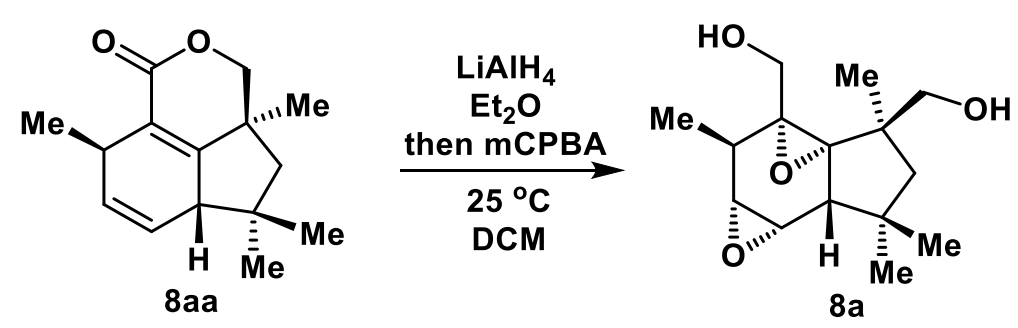

To a solution of compound $8 \mathbf{a a}\left(50 \mathrm{mg}, 0.22 \mathrm{mmol}, 1.0\right.$ equiv) in $\mathrm{Et}_{2} \mathrm{O}(30 \mathrm{~mL})$ at $0{ }^{\circ} \mathrm{C}$ was added $\mathrm{LiAlH}_{4}\left(12 \mathrm{mg}, 0.32 \mathrm{mmol}, 1.5\right.$ equiv). After being stirred at $0{ }^{\circ} \mathrm{C}$ for $2 \mathrm{~h}$, the reaction was quenched by dropwise addition of a sodium hydroxide solution $(3 \mathrm{M}, 3 \mathrm{~mL})$. The mixture was filtered through a celite pad and wash with $\mathrm{Et}_{2} \mathrm{O}(2 \times 30 \mathrm{~mL})$. The organic layers were dried over anhydrous sodium sulfate, filtered and concentrated under reduced pressure.

Without further purification, the above crude product was dissolved in dichloromethane $(30 \mathrm{~mL})$. Then mCPBA (70\%, $1.78 \mathrm{~g}, 7.22 \mathrm{mmol})$ was added to this solution. The reaction mixture was stirred at $25{ }^{\circ} \mathrm{C}$ for $12 \mathrm{~h}$. Then the mixture was quenched with a saturated aqueous solution of $\mathrm{Na}_{2} \mathrm{~S}_{2} \mathrm{O}_{3}(10 \mathrm{~mL})$, extracted with dichloromethane $(3 \times 30 \mathrm{~mL})$. The combined organic phases 
were dried over anhydrous sodium sulfate, filtered and concentrated under reduced pressure. The crude product was purified by silica gel column chromatography (hexane/ethyl acetate $=5 / 1$ ) to give $\mathbf{8 a}$ (20 mg, 35\% yield) as a light yellow oil.

$\mathbf{R}_{f}=0.25$ (hexane/ethyl acetate $\left.=2 / 1\right) ;$

${ }^{1}$ H NMR (500 MHz, CDCl $) \delta 4.00(\mathrm{~d}, J=11.7 \mathrm{~Hz}, 1 \mathrm{H}), 3.57(\mathrm{~d}, J=11.4 \mathrm{~Hz}, 1 \mathrm{H}), 3.51(\mathrm{~d}, J=$ $11.7 \mathrm{~Hz}, 1 \mathrm{H}), 3.25(\mathrm{~d}, J=11.4 \mathrm{~Hz}, 1 \mathrm{H}), 3.14(\mathrm{t}, J=3.8 \mathrm{~Hz}, 1 \mathrm{H}), 3.08(\mathrm{~d}, J=3.9 \mathrm{~Hz}, 1 \mathrm{H}), 2.76-$ $2.72(\mathrm{~m}, 1 \mathrm{H}), 2.45(\mathrm{~s}, 1 \mathrm{H}), 1.83(\mathrm{~d}, J=12.9 \mathrm{~Hz}, 1 \mathrm{H}), 1.39(\mathrm{~d}, J=12.9 \mathrm{~Hz}, 1 \mathrm{H}), 1.31(\mathrm{~s}, 3 \mathrm{H}), 1.30$ $(\mathrm{d}, J=7.0 \mathrm{~Hz}, 3 \mathrm{H}), 1.16(\mathrm{~s}, 3 \mathrm{H}), 1.04(\mathrm{~s}, 3 \mathrm{H})$;

${ }^{13}$ C NMR (125 MHz, $\left.\mathbf{C D C l}_{3}\right) \delta$ 70.5, 66.2, 60.9, 55.8, 52.9, 51.9, 49.6, 42.2, 37.3, 27.8, 27.8, 23.7, 23.3, 11.6;

HRMS (ESI) calcd for $\mathrm{C}_{15} \mathrm{H}_{24} \mathrm{O}_{4} \mathrm{Na}$ [M+Na] ${ }^{+}$Exact Mass: 291.1567; found: 291.1566.

Synthesis of 8 and 16 by the thermal IMDA reaction<smiles>C/C=C\C=C\C(C)(C)CC(C)(C#CC(=O)OCC)CO[Mg]</smiles>

9

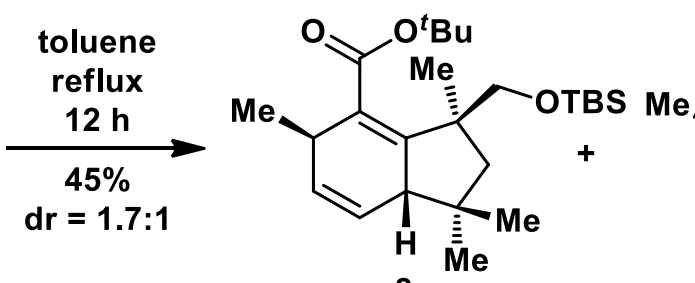

8

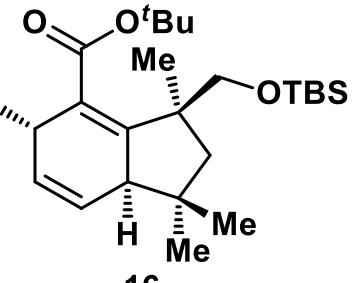

16

Compound $9(1.0 \mathrm{~g}, 7.1 \mathrm{mmol})$ was dissolved in toluene under nitrogen atmosphere and reflux for $12 \mathrm{~h}$. Then the mixture was directly concentrated under reduced pressure. The crude product was purified by silica gel column chromatography (hexane/ethyl acetate $=20 / 1)$ to give $\mathbf{8}(0.28 \mathrm{~g}, 28 \%)$ as a light yellow solid and $\mathbf{1 6}(0.17 \mathrm{~g}, 17 \%$ yield $)$ as a light yellow oil.

\section{Synthesis of 16a and 16aa via the thermal IMDA reaction}
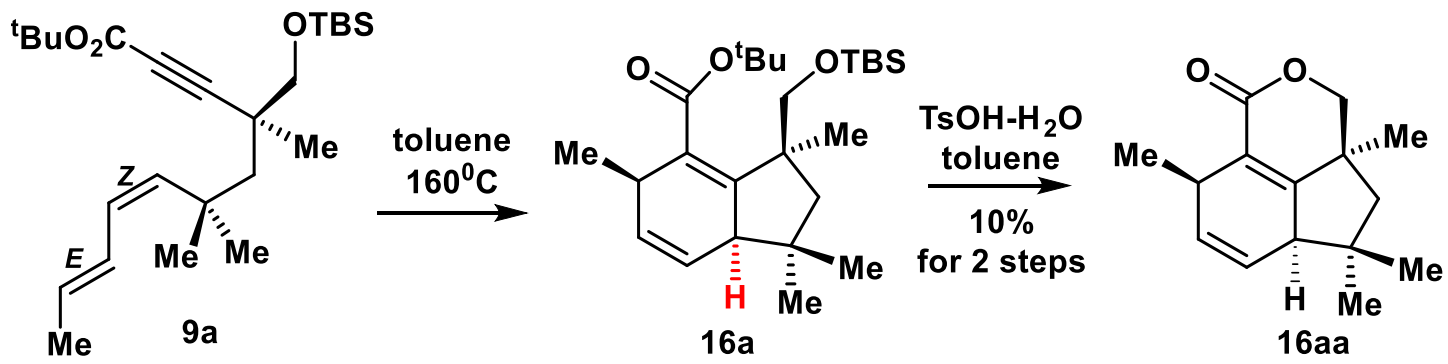

Compound 9a (380 mg, $0.86 \mathrm{mmol})$ was dissolved in toluene in a sealed tube under nitrogen 
atmosphere and was stirred at $160{ }^{\circ} \mathrm{C}$ for for $12 \mathrm{~h}$. Then the mixture was directly concentrated under reduced pressure. The crude product was purified by silica gel column chromatography (hexane/ethyl acetate $=20 / 1$ ) to give a crude product of 16 a (about $50 \mathrm{mg}$ ), which was used directly for the next step.

The crude product of $16 \mathbf{a}(50 \mathrm{mg})$ and $\mathrm{TsOH}-\mathrm{H}_{2} \mathrm{O}(20 \mathrm{mg}, 0.1 \mathrm{mmol})$ were dissolved in toluene $(100 \mathrm{~mL})$ under nitrogen atmosphere and the solution was heated to $100{ }^{\circ} \mathrm{C}$ for $24 \mathrm{~h}$. When the reaction was cooled to room temperature, the mixture was neutralized with a saturated aqueous solution of $\mathrm{NaHCO}_{3}(30 \mathrm{~mL})$, extracted with ethyl acetate $(3 \times 100 \mathrm{~mL})$. The combined organic phases were dried over anhydrous sodium sulfate, filtered and concentrated under reduced pressure. The crude product was purified by silica gel column chromatography (hexane/ethyl acetate $=10 / 1)$ to give $\mathbf{1 6 a a}(20 \mathrm{mg}, 10 \%$ yield for 2 steps $)$ as a light yellow oil.

\section{Compound 16aa}

$\mathbf{R}_{f}=0.5$ (hexane/ethyl acetate $=5 / 1$ );

IR (film) $\lambda_{\max } 3742,3626,2955,2870,1721,1573,1458,1366,1242,1049$;

${ }^{1}$ H NMR (400 MHz, CDCl $)$ ) $\delta 5.79-5.67(\mathrm{~m}, 1 \mathrm{H}), 5.60-5.50(\mathrm{~m}, 1 \mathrm{H}), 4.04(\mathrm{dd}, J=26.9,10.4$ $\mathrm{Hz}, 2 \mathrm{H}), 3.16-3.14(\mathrm{~m}, 1 \mathrm{H}), 3.05-2.86(\mathrm{~m}, 1 \mathrm{H}), 1.57(\mathrm{~d}, J=13.8 \mathrm{~Hz}, 1 \mathrm{H}), 1.46(\mathrm{~d}, J=13.8 \mathrm{~Hz}$, 1H), $1.25(\mathrm{~s}, 3 \mathrm{H}), 1.19(\mathrm{~s}, 3 \mathrm{H}), 1.16(\mathrm{~d}, J=7.1 \mathrm{~Hz}, 3 \mathrm{H}), 0.75(\mathrm{~s}, 3 \mathrm{H})$;

${ }^{13}$ C NMR (100 MHz, $\left.\mathbf{C D C l}_{3}\right) \delta 163.6,162.1,133.7,121.4,119.7,76.9,49.7,48.3,41.2,37.6$, $29.2,28.6,25.0,23.5,20.7$

HRMS (ESI) calcd for $\mathrm{C}_{15} \mathrm{H}_{21} \mathrm{O}_{2}[\mathrm{M}+\mathrm{H}]^{+}$Exact Mass: 233.1536; found: 233.1535.

\section{Synthesis of 16b}<smiles>CC1C=C[C@H]2C3=C(C(=O)OC[C@@]3(C)C[C@H]2C)C1C</smiles>

16aa

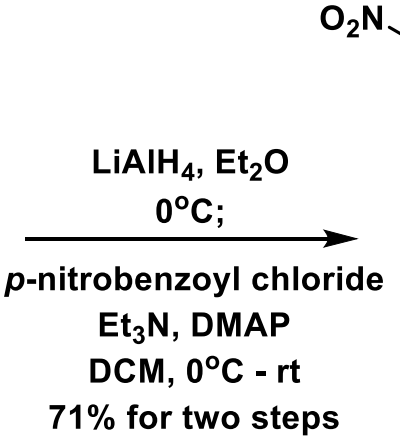

To a solution of compound 16aa $\left(60 \mathrm{mg}, 0.26 \mathrm{mmol}, 1.0\right.$ equiv) in $\mathrm{Et}_{2} \mathrm{O}(50 \mathrm{~mL})$ at $0{ }^{\circ} \mathrm{C}$ was added $\mathrm{LiAlH}_{4}\left(15 \mathrm{mg}, 0.39 \mathrm{mmol}, 1.5\right.$ equiv). After being stirred at $0{ }^{\circ} \mathrm{C}$ for $2 \mathrm{~h}$, the reaction was 
quenched by dropwise addition of a sodium hydroxide solution $(3 \mathrm{M}, 6 \mathrm{~mL})$. The mixture was filtered through a celite pad and wash with $\mathrm{Et}_{2} \mathrm{O}(2 \times 50 \mathrm{~mL})$. The organic layers were dried over anhydrous sodium sulfate, filtered and concentrated under reduced pressure.

Without further purification, the above crude product, $\mathrm{Et}_{3} \mathrm{~N}(0.18 \mathrm{~mL}, 1.30 \mathrm{mmol}, 5.0$ equiv), DMAP (10 mg), and p-nitrobenzoyl chloride (240 mg, $1.30 \mathrm{mmol}, 5.0$ equiv), were added into $\mathrm{CH}_{2} \mathrm{Cl}_{2}(25 \mathrm{~mL})$ at $0{ }^{\circ} \mathrm{C}$. The reaction mixture was allowed to warm to ambient temperature and stirred for $1 \mathrm{~h}$. The mixture was concentrated under reduced pressure and purified by silica gel column chromatography (hexane/ethyl acetate $=10 / 1)$ to give $\mathbf{1 6 b}(97 \mathrm{mg}, 71 \%$ yield) as a white solid

$\mathbf{R}_{f}=0.60$ (hexane/ethyl acetate $=5 / 1$ );

${ }^{1}$ H NMR (400 MHz, $\left.\mathbf{C D C l}_{3}\right) \delta 8.30-8.25(\mathrm{~m}, 4 \mathrm{H}), 8.24-8.11(\mathrm{~m}, 4 \mathrm{H}), 5.73-5.55(\mathrm{~m}, 2 \mathrm{H})$, $5.18(\mathrm{~d}, J=12.2 \mathrm{~Hz}, 1 \mathrm{H}), 4.96(\mathrm{~d}, J=12.2 \mathrm{~Hz}, 1 \mathrm{H}), 4.51(\mathrm{~d}, J=11.1 \mathrm{~Hz}, 1 \mathrm{H}), 4.34(\mathrm{~d}, J=11.1$ $\mathrm{Hz}, 1 \mathrm{H}), 3.11-2.95(\mathrm{~m}, 1 \mathrm{H}), 2.89(\mathrm{~d}, J=9.9 \mathrm{~Hz}, 1 \mathrm{H}), 2.02(\mathrm{~d}, J=13.6 \mathrm{~Hz}, 1 \mathrm{H}), 1.57(\mathrm{~d}, J=13.6$ $\mathrm{Hz}, 1 \mathrm{H}), 1.21(\mathrm{~d}, J=7.1 \mathrm{~Hz}, 3 \mathrm{H}), 1.13(\mathrm{~s}, 3 \mathrm{H}), 0.80(\mathrm{~s}, 3 \mathrm{H})$.

${ }^{13}$ C NMR (100 MHz, $\left.\mathbf{C D C l}_{3}\right) \delta 164.6,164.5,150.5,146.4,135.4,135.4,132.1,130.7,130.6,127.0$, 123.6, 123.6, 121.9, 72.2, 63.5, 53.0, 51.9, 43.5, 38.1, 34.4, 27.7, 25.2, 23.7, 21.0;

HRMS (ESI) calcd for $\mathrm{C}_{29} \mathrm{H}_{30} \mathrm{~N}_{2} \mathrm{NaO}_{8}[\mathrm{M}+\mathrm{Na}]^{+}$Exact Mass: 557.1894; found: 557.1895 .

\section{Synthesis of 2}
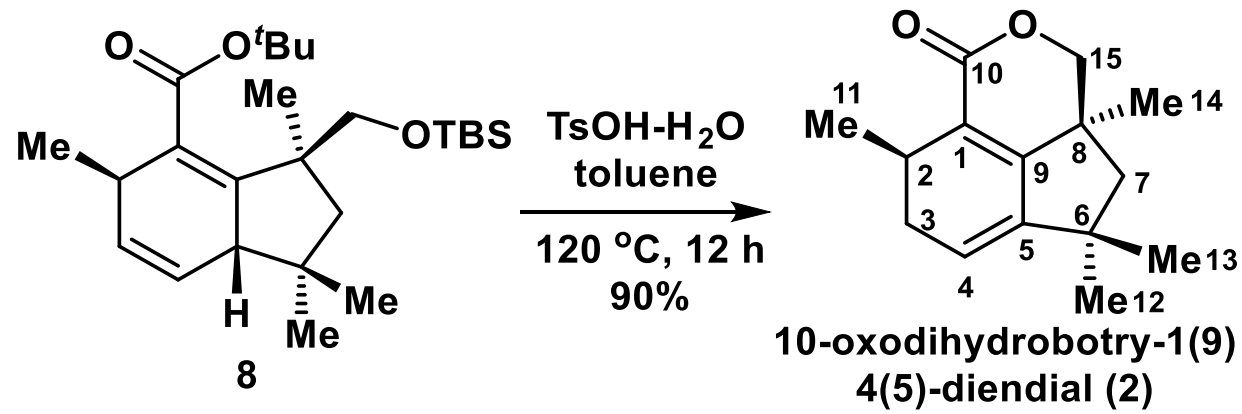

Compound 8 (1.8 g, 4.3 mmol, 1.0 equiv) and $\mathrm{TsOH}-\mathrm{H}_{2} \mathrm{O}$ (24.6 g, 12.9 mmol, 3.0 equiv) were dissolved in toluene $(100 \mathrm{~mL})$ under nitrogen atmosphere and the solution was heated to $120^{\circ} \mathrm{C}$ for $12 \mathrm{~h}$. When the reaction was cooled to $25{ }^{\circ} \mathrm{C}$, the mixture was neutralized with a saturated aqueous solution of $\mathrm{NaHCO}_{3}(100 \mathrm{~mL})$, extracted with ethyl acetate $(3 \times 200 \mathrm{~mL})$. The combined organic phases were dried over anhydrous sodium sulfate, filtered and concentrated under reduced pressure. The crude product was purified by silica gel column chromatography (hexane/ethyl 
acetate $=5 / 1)$ to give 10-oxodihydrobotry-1(9),4(5)-diendial $2(900 \mathrm{mg}, 90 \%$ yield $)$ as a light yellow solid.

Note: Using the described route, a total of $10.8 \mathrm{~g}$ of $\mathbf{2}$ was prepared readily after one dozen of simple parallel operations.

$\mathbf{R}_{f}=0.45$ (hexane/ethyl acetate $=5 / 1$ );

$[\boldsymbol{\alpha}]_{\mathbf{D}}^{24}=+17.1\left(\mathrm{c}=1.0\right.$ in $\left.\mathrm{CHCl}_{3}\right),\left[\right.$ lit. $\left\{[\boldsymbol{\alpha}]_{\mathbf{D}}^{25}=+25\left(\mathrm{c}=0.06\right.\right.$ in $\left.\left.\left.\mathrm{CHCl}_{3}\right)\right\}\right] ;$

IR (film) $\lambda_{\max }$ 2924, 2862, 1712, 1458, 1381, 1203, 1003;

${ }^{1}$ H NMR (300 MHz, CDCl $) \delta 5.73(\mathrm{dd}, J=6.4,2.6 \mathrm{~Hz}, 1 \mathrm{H}), 4.22(\mathrm{~d}, J=10.3 \mathrm{~Hz}, 1 \mathrm{H}), 3.99(\mathrm{~d}, J$ $=10.3 \mathrm{~Hz}, 1 \mathrm{H}), 2.90-2.71(\mathrm{~m}, 1 \mathrm{H}), 2.66-2.56(\mathrm{~m}, 1 \mathrm{H}), 2.25(\mathrm{dd}, J=17.6,6.4 \mathrm{~Hz}, 1 \mathrm{H}), 1.73(\mathrm{~d}$, $J=13.1 \mathrm{~Hz}, 1 \mathrm{H}), 1.52(\mathrm{~d}, J=13.1 \mathrm{~Hz}, 1 \mathrm{H}), 1.36(\mathrm{~s}, 3 \mathrm{H}), 1.28(\mathrm{~s}, 3 \mathrm{H}), 1.18(\mathrm{~s}, 3 \mathrm{H}), 0.88(\mathrm{~d}, J=$ $7.0 \mathrm{~Hz}, 3 \mathrm{H})$;

${ }^{13}$ C NMR (100 MHz, $\left.\mathbf{C D C l}_{3}\right) \delta 165.2,158.8,145.8,121.4,119.8,78.6,51.0,41.7,38.9,31.7$, $31.0,31.0,23.1,23.0,17.9$;

HRMS (ESI) calcd for $\mathrm{C}_{15} \mathrm{H}_{21} \mathrm{O}_{2}[\mathrm{M}+\mathrm{H}]^{+}$Exact Mass: 233.1536; found: 233.1539. 
Table S1 Compared NMR data $\left[\mathrm{CDCl}_{3}\right]$ between our synthetic $\mathbf{2}$ and the isolated natural product.

\begin{tabular}{|c|c|c|c|c|c|c|}
\hline \multicolumn{4}{|c|}{${ }^{1} H \& p p m(J)$} & \multicolumn{3}{|c|}{${ }^{13} \mathrm{C} \& \mathrm{ppm}$} \\
\hline Carbon & $\begin{array}{c}\text { isolated } \\
(400 \mathrm{M})\end{array}$ & $\begin{array}{c}\text { synthesized } \\
(300 \mathrm{M})\end{array}$ & $\begin{array}{c}\text { error } \\
\text { (iso. - syn.) }\end{array}$ & $\begin{array}{r}\text { isolated } \\
(100 \mathrm{M})\end{array}$ & $\begin{array}{r}\text { synthesized } \\
(100 \mathrm{M}) \\
\end{array}$ & $\begin{array}{c}\text { error } \\
\text { (iso. - syn.) }\end{array}$ \\
\hline 1 & & & & 120.1 & 119.8 & 0.3 \\
\hline 2 & 2.79 (ddq, J = 9.8, 1.2, 7.1 Hz) & $2.90-2.71(m, 1 \mathrm{H})$ & 0 & 23.3 & 23.1 & 0.2 \\
\hline 3 & $\begin{array}{l}2.24(\mathrm{ddd}, \mathrm{J}=1.2,17.6,6.4 \mathrm{~Hz}) \\
2.60(\mathrm{ddd}, \mathrm{J}=9.8,17.6,2.8 \mathrm{~Hz})\end{array}$ & $\begin{array}{l}2.25(\mathrm{dd}, \mathrm{J}=17.6,6.4 \mathrm{~Hz}, 1 \mathrm{H}) \\
2.66-2.56(\mathrm{~m}, 1 \mathrm{H})\end{array}$ & $\begin{array}{r}-0.01 \\
0\end{array}$ & 31.8 & 31.7 & 0.1 \\
\hline 5 & & & & 145.9 & 145.8 & 0.1 \\
\hline 6 & & & & 39.1 & 38.9 & 0.2 \\
\hline 7 & $\begin{array}{l}1.53(1 \mathrm{H}, \mathrm{J}=13.1 \mathrm{~Hz}) \\
1.72(1 \mathrm{H}, \mathrm{J}=13.1 \mathrm{~Hz})\end{array}$ & $\begin{array}{l}1.52(\mathrm{~d}, \mathrm{~J}=13.1 \mathrm{~Hz}, 1 \mathrm{H}) \\
1.73(\mathrm{~d}, \mathrm{~J}=13.1 \mathrm{~Hz}, 1 \mathrm{H})\end{array}$ & $\begin{array}{r}0.01 \\
-0.01\end{array}$ & 51.2 & 51.0 & 0.2 \\
\hline 8 & & & & 41.8 & 41.7 & 0.1 \\
\hline 9 & & & & 158.9 & 158.8 & 0.1 \\
\hline 14 & $1.35(\mathrm{~s})$ & $1.36(\mathrm{~s}, 3 \mathrm{H})$ & -0.01 & 23.2 & 23.0 & 0.2 \\
\hline 15 & $\begin{array}{l}3.98(\mathrm{~d}, \mathrm{~J}=10.0 \mathrm{~Hz}) \\
4.21(\mathrm{~d}, \mathrm{~J}=10.0 \mathrm{~Hz})\end{array}$ & $\begin{array}{l}3.99(\mathrm{~d}, \mathrm{~J}=10.3 \mathrm{~Hz}, 1 \mathrm{H}) \\
4.22(\mathrm{~d}, \mathrm{~J}=10.3 \mathrm{~Hz}, 1 \mathrm{H})\end{array}$ & $\begin{array}{l}-0.01 \\
-0.01\end{array}$ & 78.8 & 78.6 & 0.2 \\
\hline
\end{tabular}

\section{Synthesis of 5}
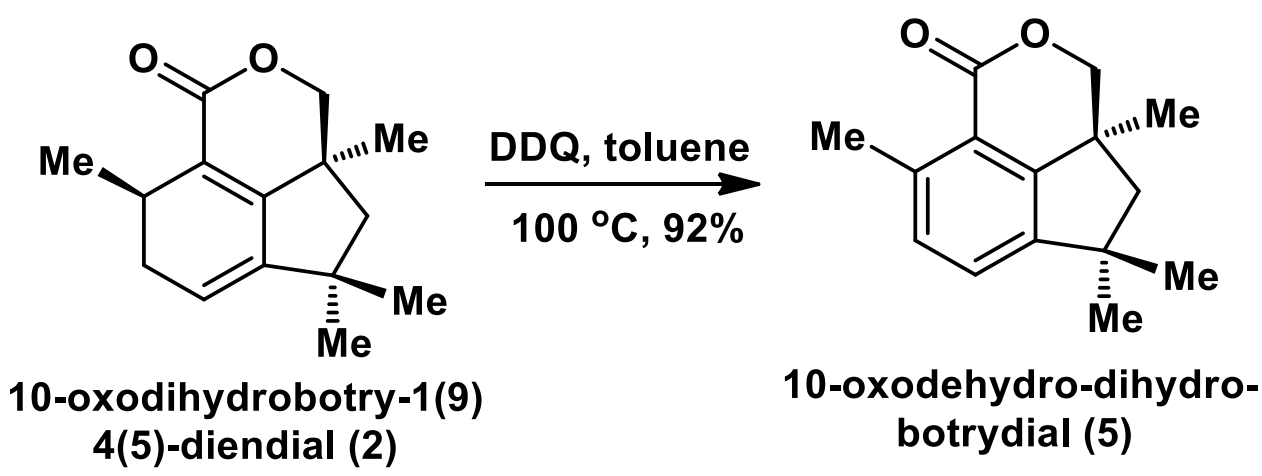

Compound 2 (50 mg, $0.21 \mathrm{mmol}, 1.0$ equiv) and DDQ (95 mg, $0.42 \mathrm{mmol}, 2.0$ equiv) were dissolved in anhydrous toluene $(30 \mathrm{~mL})$ under nitrogen atmosphere and the solution was heated to $100{ }^{\circ} \mathrm{C}$ for $12 \mathrm{~h}$. When it was cooled to $25{ }^{\circ} \mathrm{C}$, the mixture was concentrated under reduced pressure. The crude product was purified by silica gel column chromatography (hexane/ethyl acetate $=10 / 1)$ to give 10-oxodehydro-dihydro-botrydial $5(46 \mathrm{mg}, 92 \%$ yield $)$ as a light yellow solid.

$\mathbf{R}_{f}=0.45$ (hexane/ethyl acetate $\left.=10 / 1\right) ;$

$[\boldsymbol{\alpha}]_{\mathbf{D}}^{28}=-166.5\left(\mathrm{c}=1.0\right.$ in $\left.\mathrm{CHCl}_{3}\right),[\boldsymbol{\alpha}]_{\mathbf{D}}^{27}=-160.0\left(\mathrm{c}=0.39\right.$ in $\left.\mathrm{CHCl}_{3}\right),\left[\right.$ lit. $\left\{[\boldsymbol{\alpha}]_{\mathbf{D}}^{28}=+87(\mathrm{c}=\right.$ 0.39 in $\mathrm{CHCl}_{3}$ )\}]; 
IR (film) $\lambda_{\max } 2963,1759,1728,1581,1458,1373,1242,1064$

${ }^{1}$ H NMR $\left(400 \mathrm{MHz}, \mathbf{C D C l}_{3}\right) \delta 7.22(\mathrm{~d}, J=7.7 \mathrm{~Hz}, 1 \mathrm{H}), 7.15(\mathrm{~d}, J=7.7 \mathrm{~Hz}, 1 \mathrm{H}), 4.36(\mathrm{~d}, J=10.1$ $\mathrm{Hz}, 1 \mathrm{H}), 4.13(\mathrm{~d}, J=10.1 \mathrm{~Hz}, 1 \mathrm{H}), 2.62(\mathrm{~s}, 3 \mathrm{H}), 1.98(\mathrm{~d}, J=12.9 \mathrm{~Hz}, 1 \mathrm{H}), 1.86(\mathrm{~d}, J=12.9 \mathrm{~Hz}$, $1 \mathrm{H}), 1.51(\mathrm{~s}, 3 \mathrm{H}), 1.45(\mathrm{~s}, 3 \mathrm{H}), 1.32(\mathrm{~s}, 3 \mathrm{H})$;

${ }^{13}$ C NMR (75 MHz, $\mathbf{C D C l}_{3}$ ) $\delta$ 164.0, 151.4, 147.0, 139.6, 131.7, 127.4, 119.6, 79.2, 52.0, 45.1, $40.8,30.8,30.7,24.7,20.3$

HRMS (ESI) calcd for $\mathrm{C}_{15} \mathrm{H}_{19} \mathrm{O}_{2}[\mathrm{M}+\mathrm{H}]^{+}$Exact Mass: 231.1380; found: 231.1378.

Table S3 Compared NMR data $\left[\mathrm{CDC}_{3}\right]$ between our synthetic $\mathbf{5}$ and the isolated natural product.

\begin{tabular}{|c|c|c|c|c|c|c|}
\hline \multicolumn{4}{|c|}{${ }^{1} H \& p p m(J)$} & \multicolumn{3}{|c|}{${ }^{13} \mathrm{C} \& \mathrm{ppm}$} \\
\hline Carbon & $\begin{array}{l}\text { isolated } \\
(400 \mathrm{M})\end{array}$ & $\begin{array}{l}\text { synthesized } \\
(400 \mathrm{M})\end{array}$ & $\begin{array}{c}\text { error } \\
\text { (iso. - syn.) } \\
\end{array}$ & $\begin{array}{r}\text { isolated } \\
(100 \mathrm{M})\end{array}$ & $\begin{array}{c}\text { synthesized } \\
(75 \mathrm{M})\end{array}$ & $\begin{array}{c}\text { error } \\
\text { (iso. - syn.) }\end{array}$ \\
\hline 1 & & & & 119.6 & 119.6 & $\mathbf{0}$ \\
\hline 2 & & & & 139.6 & 139.6 & 0 \\
\hline 3 & $7.20(\mathrm{~d}, \mathrm{~J}=7.8 \mathrm{~Hz})$ & $7.22(\mathrm{~d}, \mathrm{~J}=7.7 \mathrm{~Hz}, 1 \mathrm{H})$ & -0.02 & 131.7 & 131.7 & 0 \\
\hline 4 & 7.14 (d, J = $7.8 \mathrm{~Hz}$ ) & $7.15(\mathrm{~d}, \mathrm{~J}=7.7 \mathrm{~Hz}, 1 \mathrm{H})$ & -0.01 & 127.3 & 127.3 & 0 \\
\hline 5 & & & & 151.4 & 151.4 & 0 \\
\hline 6 & & & & 40.8 & 40.8 & 0 \\
\hline 7 & $\begin{array}{l}1.84(\mathrm{~d}, \mathrm{~J}=13.0 \mathrm{~Hz}) \\
1.96(\mathrm{~d}, \mathrm{~J}=13.0 \mathrm{~Hz})\end{array}$ & $\begin{array}{l}1.86(\mathrm{~d}, \mathrm{~J}=12.9 \mathrm{~Hz}, 1 \mathrm{H}) \\
1.98(\mathrm{~d}, \mathrm{~J}=12.9 \mathrm{~Hz}, 1 \mathrm{H})\end{array}$ & $\begin{array}{l}-0.02 \\
-0.02\end{array}$ & 52.1 & 52.0 & 0.1 \\
\hline 8 & & & & 45.1 & 45.1 & 0 \\
\hline 9 & & & & 147.0 & 147.0 & 0 \\
\hline 10 & & & & 164.0 & 164.0 & 0 \\
\hline 11 & $2.61(\mathrm{~s})$ & $2.62(\mathrm{~s}, 3 \mathrm{H})$ & -0.01 & 20.3 & 20.3 & 0 \\
\hline 12 & 1.44 (s) & $1.45(\mathrm{~s}, 3 \mathrm{H})$ & -0.01 & 30.7 & 30.8 & -0.1 \\
\hline 13 & 1.31 (s) & $1.32(\mathrm{~s}, 3 \mathrm{H})$ & -0.01 & 30.7 & 30.7 & 0 \\
\hline 14 & $1.50(\mathrm{~s})$ & $1.51(\mathrm{~s}, 3 \mathrm{H})$ & -0.01 & 24.7 & 24.7 & 0 \\
\hline 15 & $4.12(\mathrm{~d}, \mathrm{~J}=10.1 \mathrm{~Hz})$ & $4.13(\mathrm{~d}, \mathrm{~J}=10.1 \mathrm{~Hz}, 1 \mathrm{H})$ & -0.01 & 79.2 & 79.2 & 0 \\
\hline & $4.35(\mathrm{~d}, \mathrm{~J}=10.1 \mathrm{~Hz})$ & $4.36(\mathrm{~d}, \mathrm{~J}=10.1 \mathrm{~Hz}, 1 \mathrm{H})$ & -0.01 & & & \\
\hline
\end{tabular}

Synthesis of 1-(2-hydroxy-6-((4-methoxybenzyl)oxy)phenyl)ethanone<smiles>CC(=O)c1c(O)cccc1O</smiles>

\section{1-(2,6-dihydroxyphenyl) ethanone}

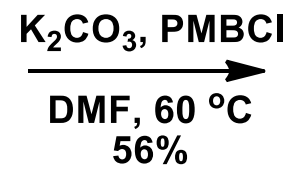<smiles>CCOc1cccc(O)c1C(C)=O</smiles>

\section{1-(2-hydroxy-6-((4-methoxybenzyl)oxy)} phenyl)ethanone

To a solution of dimethylformamide $(250 \mathrm{~mL})$ was added 1-(2,6-dihydroxyphenyl)ethanone (20.0 $\mathrm{g}, 131.6 \mathrm{mmol}, 1.0$ equiv). Potassium carbonate ( $36.3 \mathrm{~g}, 263.2 \mathrm{~mol}, 2.0$ equiv) was added in portions. The mixture was stirred for $1 \mathrm{~h}$ at $60{ }^{\circ} \mathrm{C}$. Then 4-methoxybenzyl chloride $(35.7 \mathrm{~mL}$, $263.2 \mathrm{~mol}, 2.0$ equiv) was added dropwise to the mixture and the mixture was stirred for another 3 $\mathrm{h}$ at $60{ }^{\circ} \mathrm{C}$. Water $(500 \mathrm{~mL})$ was added to quench the reaction, extracted with $\mathrm{Et}_{2} \mathrm{O}(3 \times 300 \mathrm{~mL})$. 
The organic layers were dried over anhydrous sodium sulfate, filtered and concentrated under reduced pressure, to provide a waxy yellow solid. Recrystallization of the waxy yellow solid by using dichloromethane and water for twice to give (2-hydroxy-6-((4-methoxybenzyl)oxy)phenyl)ethanone(R. J. Griffin, G. Fontana, B. T. Golding, S. Guiard, I. R. Hardcastle, J. J. J. Leahy, N. Martin, C. Richardson, L. Rigoreau, M. Stockley, G. C. M. Smith, J. Med. Chem. 2005, 48, 569.) (20.0 g, 56\% yield) as a white solid.

$\mathbf{R}_{f}=0.3$ (hexane/ethyl acetate $=5 / 1$;

IR (film) $\lambda_{\max } 2994,1767,1612,1458,1373,1242,1057,495$;

${ }^{1}$ H NMR (400 MHz, CDCl $) \delta 13.27(\mathrm{~s}, 1 \mathrm{H}), 7.32-7.36(\mathrm{~m}, 3 \mathrm{H}), 6.93(\mathrm{~d}, J=8.5 \mathrm{~Hz}, 2 \mathrm{H}), 6.58$ $(\mathrm{d}, J=8.3 \mathrm{~Hz}, 1 \mathrm{H}), 6.48(\mathrm{~d}, J=8.3 \mathrm{~Hz}, 1 \mathrm{H}), 5.05(\mathrm{~s}, 2 \mathrm{H}), 3.83(\mathrm{~s}, 3 \mathrm{H}), 2.58(\mathrm{~s}, 3 \mathrm{H})$;

${ }^{13}$ C NMR (100 MHz, $\left.\mathbf{C D C l}_{3}\right) \delta$ 205.2, 164.7, 160.7, 159.7, 136.1, 129.7, 127.7, 114.1, 111.5, $110.8,102.1,70.8,55.3,34.1$

HRMS (ESI) calcd for $\mathrm{C}_{16} \mathrm{H}_{16} \mathrm{NaO}_{4}[\mathrm{M}+\mathrm{Na}]^{+}$Exact Mass: 295.0941; found: 295.0942.

\section{Synthesis of 18}<smiles>CCCCCOc1cccc(O)c1C(C)=O</smiles>
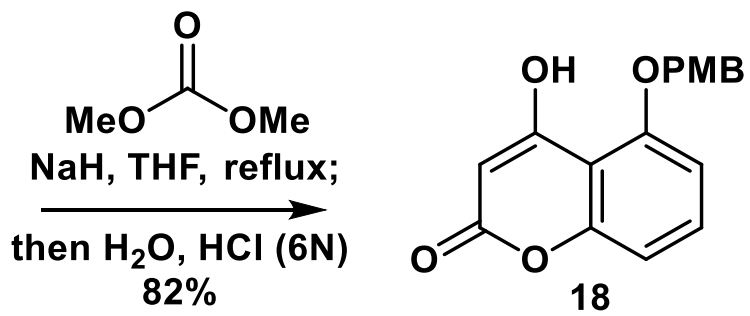

To a solution of 1-(2-hydroxy-6-((4-methoxybenzyl)oxy)phenyl)ethanone (20.0 g, $73.5 \mathrm{mmol}, 1.0$ equiv) in THF ( $250 \mathrm{~mL})$ was added $\mathrm{NaH}\left(60 \%\right.$ in oil, $23.5 \mathrm{~g}, 600 \mathrm{mmol}, 8.0$ equiv) at $25^{\circ} \mathrm{C}$. Then the solution was raise to $60{ }^{\circ} \mathrm{C}$ and stirred for $10 \mathrm{~min}$. Diethyl carbonate $(18.6 \mathrm{ml}, 220.5 \mathrm{mmol}, 3.0$ equiv) was dropwise added and the mixture was stirred at $60{ }^{\circ} \mathrm{C}$ for $3 \mathrm{~h}$. The solution was then cooled to rt and water $(250 \mathrm{~mL})$ and $\mathrm{Et}_{2} \mathrm{O}(50 \mathrm{~mL})$ was added. Then an aqueous solution of $\mathrm{HCl}(6$ $\mathrm{M}, 50 \mathrm{~mL}$ ) was added to adjust the $\mathrm{pH}$ to $1 \sim 2$. Then the mixture was extracted with dichloromethane $(3 \times 300 \mathrm{~mL})$. The organic layers were dried over anhydrous sodium sulfate, filtered and concentrated under reduced pressure. The crude product was recrystallized by using dichloromethane and water for twice to give compound $\mathbf{1 8}(18.0 \mathrm{~g}, 82 \%$ yield) as a white solid. $\mathbf{R}_{f}=0.2$ (hexane/ethyl acetate $\left.=2 / 1\right) ;$ IR (film) $\lambda_{\max } 2932,2361,1767,1721,1651,1612,1466,1242,1065,818,748$; 
${ }^{1}$ H NMR (400 MHz, $\left.\mathbf{C D C l}_{3}\right) \delta 9.65(\mathrm{~s}, 1 \mathrm{H}), 7.55-7.30(\mathrm{~m}, 3 \mathrm{H}), 7.08-6.83(\mathrm{~m}, 4 \mathrm{H}), 5.62(\mathrm{~s}$, $1 \mathrm{H}), 5.18(\mathrm{~s}, 2 \mathrm{H}), 3.83(\mathrm{~s}, 3 \mathrm{H})$;

${ }^{13}$ C NMR (100 MHz, $\left.\mathbf{C D C l}_{3}\right) \delta 166.0,160.4,155.4,155.0,132.5,130.0,125.8,114.5,111.3$, $106.8,105.1,99.9,92.8,72.5,55.3$

HRMS (ESI) calcd for $\mathrm{C}_{17} \mathrm{H}_{14} \mathrm{NaO}_{5}[\mathrm{M}+\mathrm{Na}]^{+}$Exact Mass: 321.0733; found: 321.0735 .

\section{Synthesis of 19a and 19b}<smiles>CC1CC=C2C3=C1C(=O)OC[C@]3(C)CC2(C)C</smiles>

10-oxodihydrobotry-1(9), 4(5)-diendial (2)<smiles>COc1cccc2oc(=O)cc(O)c12</smiles>

17

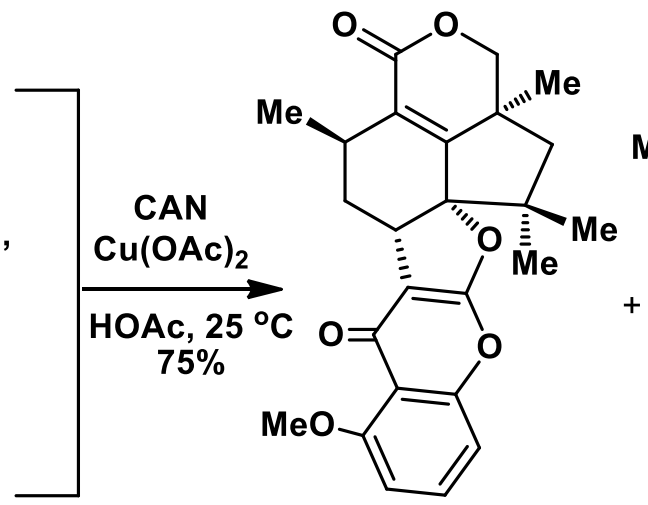

$19 a$

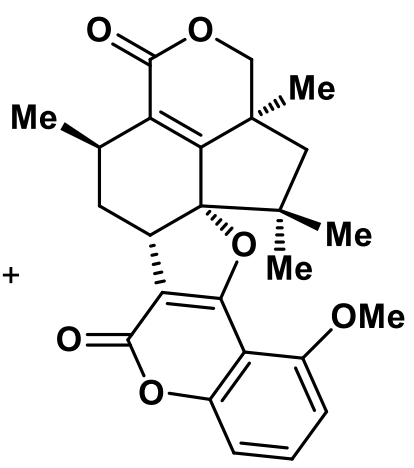

$19 b$

$(19 b / 19 a=1)$

A solution of compound $2(300 \mathrm{mg}, 1.27 \mathrm{mmol}, 1.0$ equiv) and compound 17 (488 mg, $2.54 \mathrm{mmol}$, 2.0 equiv) in HOAc $(50 \mathrm{~mL})$ was bubbled nitrogen for $0.5 \mathrm{~h}$. Then CAN $(2.09 \mathrm{~g}, 3.81 \mathrm{mmol}, 3.0$ equiv) and $\mathrm{Cu}(\mathrm{OAc})_{2}(694 \mathrm{mg}, 3.82 \mathrm{mmol}, 3.0$ equiv) were added to this solution under the protection of dry nitrogen. The reaction mixture was stirred for $12 \mathrm{~h}$ at $25{ }^{\circ} \mathrm{C}$, then was diluted with water $(100 \mathrm{~mL})$ and extracted with dichloromethane $(3 \mathrm{x} 80 \mathrm{~mL})$. The combined organic phases was diluted with $\mathrm{H}_{2} \mathrm{O}(100 \mathrm{~mL})$ and neutralized with solid $\mathrm{K}_{2} \mathrm{CO}_{3}(20 \mathrm{~g})$. The mixture was filtered through a celite pad and extracted with $\mathrm{CH}_{2} \mathrm{Cl}_{2}(3 \times 80 \mathrm{~mL})$. The organic layers were dried over anhydrous sodium sulfate, filtered and concentrated under reduced pressure. The crude product was purified by silica gel column chromatography (hexane/ethyl acetate $=3 / 1$ ) to give compound 19a (200 mg, 37\% yield) as a white solid and compound 19b (205 mg, 38\% yield) as a white solid.

\section{Compound 19a}

$\mathbf{R}_{f}=0.15$ (hexane/ethyl acetate $=2 / 1$ ); 
IR (film) $\lambda_{\max } 3672,2924,1720,1065,1466,1389,1242,1056,918,871,748$;

${ }^{1}$ H NMR (400 MHz, CDCl $) \delta 7.49(\mathrm{t}, J=8.4 \mathrm{~Hz}, 1 \mathrm{H}), 7.00(\mathrm{dd}, J=8.4,0.9 \mathrm{~Hz}, 1 \mathrm{H}), 6.86(\mathrm{~d}, J$ $=8.3 \mathrm{~Hz}, 1 \mathrm{H}), 4.13(\mathrm{q}, J=10.5 \mathrm{~Hz}, 2 \mathrm{H}), 3.98(\mathrm{~s}, 3 \mathrm{H}), 3.57(\mathrm{dd}, J=7.8,5.9 \mathrm{~Hz}, 1 \mathrm{H}), 2.91-2.75$ (m, 1H), $2.07-2.02(\mathrm{~m}, 2 \mathrm{H}), 1.95(\mathrm{~d}, J=13.8 \mathrm{~Hz}, 1 \mathrm{H}), 1.57(\mathrm{~d}, J=13.8 \mathrm{~Hz}, 1 \mathrm{H}), 1.45(\mathrm{~s}, 3 \mathrm{H})$, $1.14(\mathrm{~s}, 3 \mathrm{H}), 1.12(\mathrm{~d}, J=7.1 \mathrm{~Hz}, 3 \mathrm{H}), 1.03(\mathrm{~s}, 3 \mathrm{H})$;

${ }^{13}$ C NMR (100 MHz, $\left.\mathbf{C D C l}_{3}\right) \delta 175.1,165.9,163.1,160.2,155.7,154.5,132.4,131.1,114.3$, $109.9,107.8,99.8,98.8,77.6,56.5,47.9,45.2,37.4,34.0,33.3,26.3,25.4,24.1,21.9,18.2$;

HRMS (ESI) calcd for $\mathrm{C}_{25} \mathrm{H}_{27} \mathrm{O}_{6}[\mathrm{M}+\mathrm{H}]^{+}$Exact Mass: 423.1802; found: 423.1804 .

\section{Compound 19b}

$\mathbf{R}_{f}=0.4$ (hexane/ethyl acetate $=2 / 1$ );

IR (film) $\lambda_{\max } 3680,2924,1759,1728,1628,1605,1450,1373,1242,1057,964,918,856,756$;

${ }^{1}$ H NMR (400 MHz, CDCl $) \delta 7.47(\mathrm{t}, J=8.4 \mathrm{~Hz}, 1 \mathrm{H}), 7.06-6.90(\mathrm{~m}, 1 \mathrm{H}), 6.73(\mathrm{~d}, J=8.2 \mathrm{~Hz}$, 1H), $4.17(\mathrm{q}, J=10.6 \mathrm{~Hz}, 2 \mathrm{H}), 3.92(\mathrm{~s}, 3 \mathrm{H}), 3.41(\mathrm{dd}, J=10.2,5.1 \mathrm{~Hz}, 1 \mathrm{H}), 2.95-2.81(\mathrm{~m}, 1 \mathrm{H})$, $2.20-2.07(\mathrm{~m}, 1 \mathrm{H}), 1.99(\mathrm{~d}, J=13.6 \mathrm{~Hz}, 1 \mathrm{H}), 1.85-1.75(\mathrm{~m}, 1 \mathrm{H}), 1.60(\mathrm{~d}, J=13.6 \mathrm{~Hz}, 1 \mathrm{H})$, $1.54(\mathrm{~s}, 3 \mathrm{H}), 1.11(\mathrm{~d}, J=7.1 \mathrm{~Hz}, 3 \mathrm{H}), 1.06(\mathrm{~s}, 3 \mathrm{H}), 1.03(\mathrm{~s}, 3 \mathrm{H})$;

${ }^{13}$ C NMR (100 MHz, $\left.\mathbf{C D C l}_{3}\right) \delta 165.6,163.3,159.9,156.7,156.5,155.1,132.8,129.8,109.7$, 106.2, 105.6, 103.4, 99.5, 77.7, 56.2, 48.3, 45.4, 37.4, 33.9, 32.4, 26.1, 25.5, 23.8, 22.6, 18.1;

HRMS (ESI) calcd for $\mathrm{C}_{25} \mathrm{H}_{27} \mathrm{O}_{6}[\mathrm{M}+\mathrm{H}]^{+}$Exact Mass: 423.1802; found: 423.1802 .

\section{Synthesis of 20a and 20b}

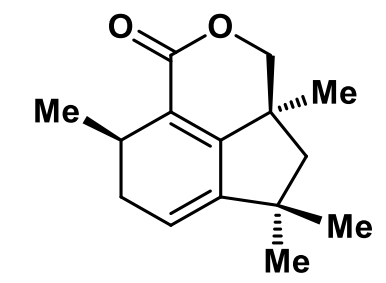

10-oxodihydrobotry-1(9), 4(5)-diendial (2)<smiles>CCCCOc1cccc2oc(=O)cc(O)c12</smiles>

18

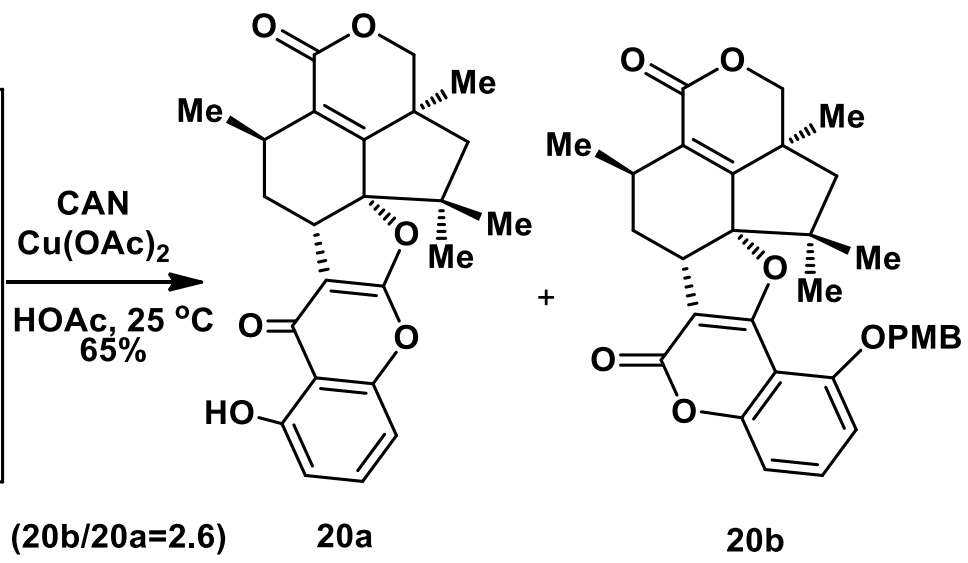


A solution of 2 (500 mg, $2.12 \mathrm{mmol}, 1.0$ equiv) and $\mathbf{1 8}$ (1.26 g, $4.22 \mathrm{mmol}, 2.0$ equiv) in HOAc $(120 \mathrm{~mL})$ in HOAc $(90 \mathrm{~mL})$ was bubbled nitrogen for $0.5 \mathrm{~h}$. Then CAN $(3.49 \mathrm{~g}, 6.36 \mathrm{mmol}, 3.0$ equiv) and $\mathrm{Cu}(\mathrm{OAc})_{2}(1.16 \mathrm{~g}, 6.36 \mathrm{mmol}, 3.0$ equiv) were added to this solution under the protection of dry nitrogen. The reaction mixture was stirred for $12 \mathrm{~h}$ at $25{ }^{\circ} \mathrm{C}$ then was diluted with water $(200 \mathrm{~mL})$ and extracted with dichloromethane $(3 \times 100 \mathrm{~mL})$. The combined organic phases was diluted with $\mathrm{H}_{2} \mathrm{O}(100 \mathrm{~mL})$ and neutralized with solid $\mathrm{K}_{2} \mathrm{CO}_{3}(40 \mathrm{~g})$. The mixture was filtered through a Celite pad and extracted with $\mathrm{CH}_{2} \mathrm{Cl}_{2}(3 \times 100 \mathrm{~mL})$. The organic layers were dried over anhydrous sodium sulfate, filtered and concentrated under reduced pressure. The crude product was purified by silica gel column chromatography (hexane/ethyl acetate $=3 / 1)$ to give 20a $(200$ $\mathrm{mg}, 18 \%$ yield) as a white solid and $\mathbf{2 0 b}$ (520 $\mathrm{mg}, 47 \%$ yield) as a white solid.

\section{Compound 20a}

$\mathbf{R}_{f}=49.2$ (hexane/ethyl acetate $\left.=2 / 1\right) ;$

$[\boldsymbol{\alpha}]_{\mathbf{D}}^{\mathbf{2 6}}=-189.3\left(\mathrm{c}=1.0\right.$ in $\left.\mathrm{CHCl}_{3}\right)$;

IR (film) $\lambda_{\max } 3672,2924,1759,1573,1458,1381,1242,1049,956,856,756$;

${ }^{1}$ H NMR (400 MHz, $\left.\mathbf{C D C l}_{3}\right) \delta 12.81(\mathrm{~s}, 1 \mathrm{H}), 7.45(\mathrm{t}, J=8.3 \mathrm{~Hz}, 1 \mathrm{H}), 6.88-6.82(\mathrm{~m}, 2 \mathrm{H}), 4.18$ $(\mathrm{d}, J=10.6 \mathrm{~Hz}, 1 \mathrm{H}), 4.12(\mathrm{~d}, J=10.6 \mathrm{~Hz}, 1 \mathrm{H}), 3.58(\mathrm{dd}, J=9.8,5.0 \mathrm{~Hz}, 1 \mathrm{H}), 2.94-2.87(\mathrm{~m}, 1 \mathrm{H})$, $2.18-2.10(\mathrm{~m}, 1 \mathrm{H}), 1.97(\mathrm{~d}, J=13.8 \mathrm{~Hz}, 1 \mathrm{H}), 1.95-1.85(\mathrm{~m}, 1 \mathrm{H}), 1.61(\mathrm{~d}, J=13.8 \mathrm{~Hz}, 1 \mathrm{H})$, $1.46(\mathrm{~s}, 3 \mathrm{H}), 1.15(\mathrm{~s}, 3 \mathrm{H}), 1.13(\mathrm{~d}, J=7.1 \mathrm{~Hz}, 3 \mathrm{H}), 1.05(\mathrm{~s}, 3 \mathrm{H})$;

${ }^{13} \mathrm{C}$ NMR (100 MHz, $\mathbf{C D C l}_{3}$ ) $\delta$ 179.9, 168.3, 162.9, 161.1, 153.7, 153.66, 133.7, 131.4, 112.9, 109.7, 106.9, 99.6, 98.7, 77.6, 47.9, 45.4, 37.3, 34.2, 32.1, 26.3, 25.6, 24.0, 22.0, 18.1;

HRMS (ESI) calcd for $\mathrm{C}_{24} \mathrm{H}_{24} \mathrm{NaO}_{6}[\mathrm{M}+\mathrm{Na}]^{+}$Exact Mass: 409.1646; found: 431.1462 .

\section{Compound 20b}

$\mathbf{R}_{f}=0.30$ (hexane/ethyl acetate $=2 / 1$ );

$[\boldsymbol{\alpha}]_{\mathbf{D}}^{26}=-144.3\left(\mathrm{c}=1.0\right.$ in $\left.\mathrm{CHCl}_{3}\right)$;

IR (film) $\lambda_{\max }$ 2924, 2338, 1767, 1612, 1466, 1373, 1242, 1049, 918, 756;

${ }^{1}$ H NMR (400 MHz, CDCl $) \delta 7.47(\mathrm{t}, J=8.4 \mathrm{~Hz}, 1 \mathrm{H}), 7.35(\mathrm{~d}, J=8.5 \mathrm{~Hz}, 2 \mathrm{H}), 6.99(\mathrm{~d}, J=8.4$ Hz, 1H), $6.91(\mathrm{~d}, J=8.6 \mathrm{~Hz}, 2 \mathrm{H}), 6.83(\mathrm{~d}, J=8.4 \mathrm{~Hz}, 1 \mathrm{H}), 5.05(\mathrm{~d}, J=10.3 \mathrm{~Hz}, 1 \mathrm{H}), 4.98(\mathrm{~d}, J=$ $10.3 \mathrm{~Hz}, 1 \mathrm{H}), 4.01$ (s, 2H), 3.83 (s, 3H), 3.35 (dd, $J=10.1,5.0 \mathrm{~Hz}, 1 \mathrm{H}), 2.80-2.82(\mathrm{~m}, 1 \mathrm{H}), 2.06$ $-2.12(\mathrm{~m}, 1 \mathrm{H}), 1.82-1.72(\mathrm{~m}, 1 \mathrm{H}), 1.60(\mathrm{~d}, J=13.6 \mathrm{~Hz}, 1 \mathrm{H}), 1.35(\mathrm{~d}, J=13.6 \mathrm{~Hz}, 1 \mathrm{H}), 1.06(\mathrm{~d}$, $J=7.1 \mathrm{~Hz}, 3 \mathrm{H}), 1.03(\mathrm{~s}, 3 \mathrm{H}), 0.97(\mathrm{~s}, 3 \mathrm{H}), 0.84(\mathrm{~s}, 3 \mathrm{H})$; 
${ }^{13}$ C NMR (100 MHz, $\left.\mathbf{C D C l}_{3}\right) \delta$ 165.6, 163.3, 159.9, 156.6, 155.9, 155.1, 132.8, 130.6, 129.8, 127.7, 114.1, 109.7, 106.4, 106.2, 103.4, 99.5, 97.6, 77.6, 71.3, 55.4, 47.6, 45.4, 37.1, 33.7, 32.4, $26.1,25.4,22.8,22.5,18.1$

HRMS (ESI) calcd for $\mathrm{C}_{32} \mathrm{H}_{33} \mathrm{O}_{7}[\mathrm{M}+\mathrm{H}]^{+}$Exact Mass: 529.2221; found: 529.2224.

\section{Synthesis of 20ba}

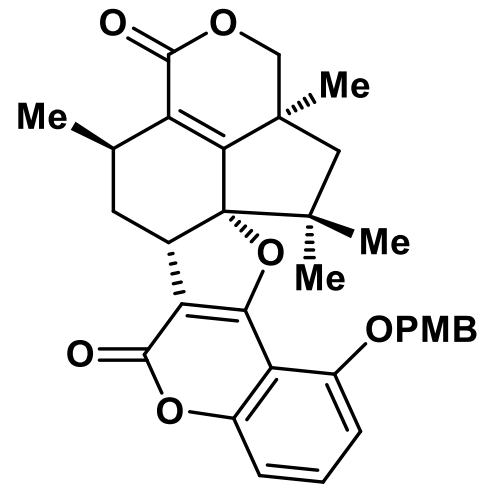

$20 \mathrm{~b}$
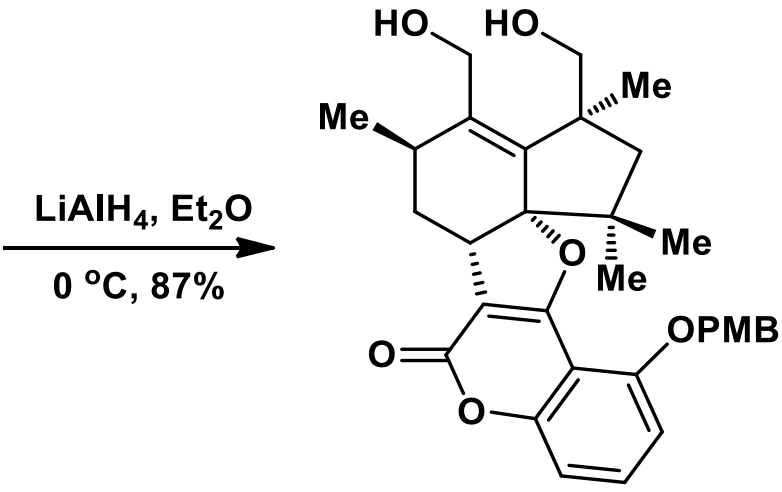

$20 \mathrm{ba}$

To a solution of $20 \mathrm{~b}\left(100 \mathrm{mg}, 0.19 \mathrm{mmol}, 1.0\right.$ equiv) in $100 \mathrm{~mL} \mathrm{Et}_{2} \mathrm{O}$ at $0{ }^{\circ} \mathrm{C}$ was added $\mathrm{LiAlH}_{4}$ (14 mg, $0.38 \mathrm{mmol}, 2.0$ equiv). After being stirred at $0{ }^{\circ} \mathrm{C}$ for $1 \mathrm{~h}$, the reaction was quenched by dropwise addition of of an aqueous sodium hydroxide solution (3 M, $6 \mathrm{~mL})$. The mixture was filtered through a Celite pad and wash with $\mathrm{Et}_{2} \mathrm{O}(2 \times 10 \mathrm{~mL})$. The organic layers were dried over anhydrous sodium sulfate, filtered and concentrated under reduced pressure. The crude product was purified by silica gel column chromatography (dichloromethane/methanol $=10 / 1$ ) to give 20ba ( $85 \mathrm{mg}, 87 \%$ yield) as a white solid.

$\mathbf{R}_{f}=0.5$ (dichloromethane/methanol $\left.=8 / 1\right)$;

$[\boldsymbol{\alpha}]_{\mathbf{D}}^{27}=-166.7\left(\mathrm{c}=0.50\right.$ in $\left.\mathrm{CHCl}_{3}\right)$;

IR (film) $\lambda_{\max }$ 2927, 2338, 1767, 1697, 1612, 1466, 1373, 1250, 1096, 1034, 895, 756;

${ }^{1} \mathbf{H}$ NMR $\left(\mathbf{5 0 0} \mathbf{~ M H z}, \mathbf{C D C l}_{\mathbf{3}}\right) \delta 7.41(\mathrm{t}, J=8.4 \mathrm{~Hz}, 1 \mathrm{H}), 7.37-7.30(\mathrm{~m}, 2 \mathrm{H}), 6.95(\mathrm{dd}, J=8.4,0.6 \mathrm{~Hz}$, 1H), $6.93-6.87(\mathrm{~m}, 2 \mathrm{H}), 6.77(\mathrm{~d}, J=8.2 \mathrm{~Hz}, 1 \mathrm{H}), 5.05(\mathrm{~d}, J=10.7 \mathrm{~Hz}, 1 \mathrm{H}), 4.98(\mathrm{~d}, J=10.7 \mathrm{~Hz}, 1 \mathrm{H})$, $4.25(\mathrm{~d}, J=11.3 \mathrm{~Hz}, 1 \mathrm{H}), 4.01(\mathrm{~d}, J=11.3 \mathrm{~Hz}, 1 \mathrm{H}), 3.83(\mathrm{~s}, 3 \mathrm{H}), 3.64(\mathrm{~d}, J=11.6 \mathrm{~Hz}, 1 \mathrm{H}), 3.56$ (dd, $J$ = 5.4, $2.3 \mathrm{~Hz}, 1 \mathrm{H}), 3.48(\mathrm{~d}, J=11.6 \mathrm{~Hz}, 1 \mathrm{H}), 2.84(\mathrm{~s}, 1 \mathrm{H}), 2.54-2.57(\mathrm{~m}, 1 \mathrm{H}), 2.28-2.33(\mathrm{~m}, 1 \mathrm{H})$, $2.22(\mathrm{~s}, 1 \mathrm{H}), 1.63(\mathrm{q}, J=13.0 \mathrm{~Hz}, 2 \mathrm{H}), 1.32-1.38(\mathrm{~m}, 1 \mathrm{H}), 1.17(\mathrm{~d}, J=7.0 \mathrm{~Hz}, 3 \mathrm{H}), 0.99(\mathrm{~s}, 6 \mathrm{H}), 0.84$ (s, 3H); 
${ }^{13}$ C NMR (125 MHz, $\left.\mathbf{C D C l}_{3}\right) \delta$ 166.7, 160.4, 159.8, 156.4, 155.9, 143.1, 141.4, 132.3, 130.1, 128.0, 114.0, 109.6, 106.4, 106.0, 104.0, 103.1, 71.6, 70.9, 59.5, 55.4, 52.2, 45.5, 41.9, 36.3, 33.7, 29.7, 25.8 , 24.1, 21.3, 18.4;

HRMS (ESI) calcd for $\mathrm{C}_{32} \mathrm{H}_{36} \mathrm{NaO}_{7}[\mathrm{M}+\mathrm{Na}]^{+}$Exact Mass: 555.2353; found: 555.2354.

\section{Synthesis of 21}

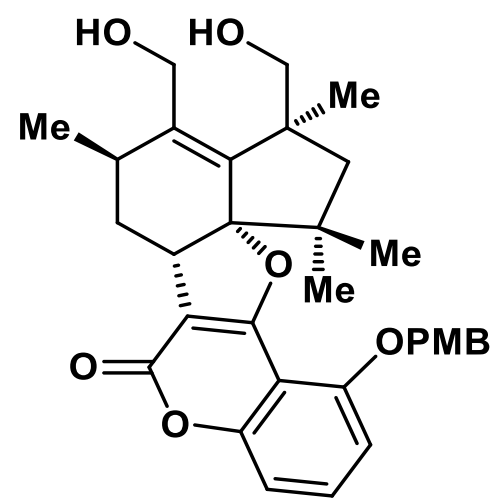

$20 \mathrm{ba}$

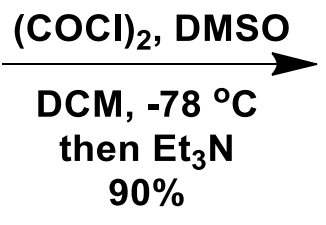

$90 \%$

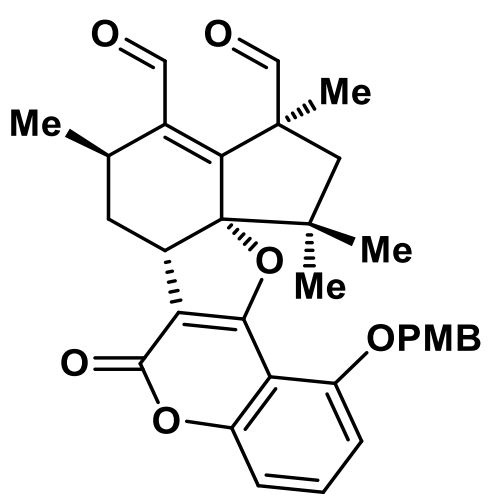

21

Oxalyl chloride ( $73 \mu \mathrm{l}, 0.87 \mathrm{mmol}, 2.3$ equiv) was added to a flask containing dichloromethane $(100 \mathrm{~mL})$ at $-78{ }^{\circ} \mathrm{C}$. Then methyl sulfoxide $(0.1 \mathrm{ml}, 1.44 \mathrm{mmol}, 3.8$ equiv) was added to this solution. After stirring at the same temperature for $0.5 \mathrm{~h}, \mathbf{2 0 b a}(200 \mathrm{mg}, 0.38 \mathrm{mmol}, 1.0$ equiv) in dichloromethane $(50 \mathrm{~mL})$ was slowly added. After stirring at $-78{ }^{\circ} \mathrm{C}$ for another $0.5 \mathrm{~h}$, the mixture was treated with triethylamine $\left(0.35 \mathrm{ml}, 2.51 \mathrm{mmol}, 6.6\right.$ equiv). The solution was stirred at $-78{ }^{\circ} \mathrm{C}$ for $0.5 \mathrm{~h}$, and then was allowed to warm to $0{ }^{\circ} \mathrm{C}$. This reaction was quenched with an aqueous solution of $\mathrm{HCl}(3 \mathrm{M}, 10 \mathrm{~mL})$, extracted with dichloromethane $(3 \times 50 \mathrm{~mL})$. The organic layers were dried over anhydrous sodium sulfate, filtered and concentrated under reduced pressure. The crude product was purified by silica gel column chromatography (hexane/ethyl acetate $=5 / 1$ ) to give 21 (180 mg, 90\% yield) as a white solid.

$\mathbf{R}_{f}=0.4$ (hexane/ethyl acetate $=3 / 1$ );

$[\boldsymbol{\alpha}]_{\mathbf{D}}^{26}=-189.5\left(\mathrm{c}=0.50\right.$ in $\left.\mathrm{CHCl}_{3}\right)$;

IR (film) $\lambda_{\max } 2361,1767,1612,1512,1366,1258,1026,756$;

${ }^{1}$ H NMR (400 MHz, CDCl $) \delta 9.58(\mathrm{~s}, 1 \mathrm{H}), 9.53(\mathrm{~s}, 1 \mathrm{H}), 7.47(\mathrm{t}, J=8.4 \mathrm{~Hz}, 1 \mathrm{H}), 7.34(\mathrm{~d}, J=8.6$ Hz, 2H), $6.99(\mathrm{~d}, J=8.3 \mathrm{~Hz}, 1 \mathrm{H}), 6.91(\mathrm{~d}, J=8.6 \mathrm{~Hz}, 2 \mathrm{H}), 6.82(\mathrm{~d}, J=8.3 \mathrm{~Hz}, 1 \mathrm{H}), 5.04(\mathrm{~d}, J=$ $10.3 \mathrm{~Hz}, 1 \mathrm{H}), 4.97(\mathrm{~d}, J=10.3 \mathrm{~Hz}, 1 \mathrm{H}), 3.83(\mathrm{~s}, 3 \mathrm{H}), 3.56(\mathrm{t}, J=5.4 \mathrm{~Hz}, 1 \mathrm{H}), 2.76-2.64(\mathrm{~m}, 1 \mathrm{H})$, 
$2.32-2.21(\mathrm{~m}, 1 \mathrm{H}), 1.79(\mathrm{~d}, J=7.6 \mathrm{~Hz}, 1 \mathrm{H}), 1.66(\mathrm{~d}, J=7.6 \mathrm{~Hz}, 1 \mathrm{H}), 1.59(\mathrm{~s}, 3 \mathrm{H}), 1.17(\mathrm{~d}, J=$ $7.0 \mathrm{~Hz}, 3 \mathrm{H}), 1.02(\mathrm{~s}, 3 \mathrm{H}), 0.99$ (s, 3H), 0.87 (s, 3H);

${ }^{13}$ C NMR (100 MHz, $\left.\mathbf{C D C l}_{3}\right) \delta$ 200.2, 191.6, 165.8, 160.0, 159.9, 156.5, 155.9, 153.3, 143.1, $132.8,130.5,127.7,114.1,109.7,106.4,104.4,103.5,103.2,71.1,55.4,53.7,49.0,43.6,34.7$, $33.2,26.3,23.5,23.1,20.7,19.1$

HRMS (ESI) calcd for $\mathrm{C}_{32} \mathrm{H}_{33} \mathrm{O}_{7}[\mathrm{M}+\mathrm{H}]^{+}$Exact Mass: 529.2221; found: 529.2219.

Synthesis of (-)-hypocrolide A (6) and (-)- $\mathrm{C}_{1,2}$-epi-hypocrolide A (22)

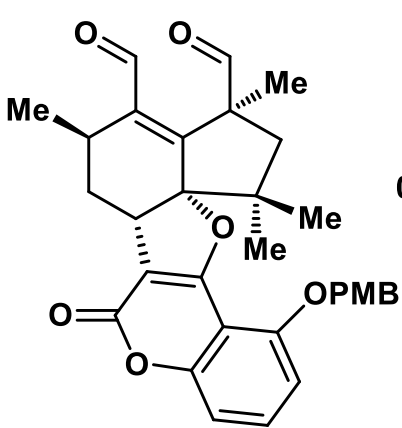

21

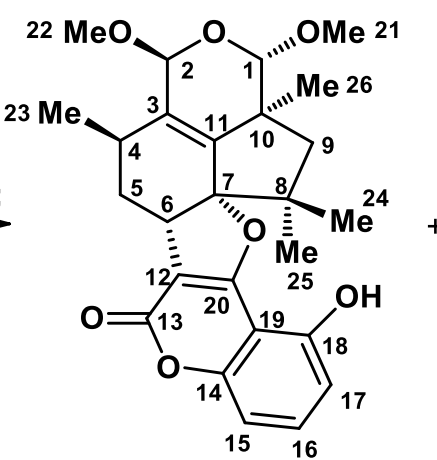

(-)-hypocrolide A (6) $>99 \%$ ee

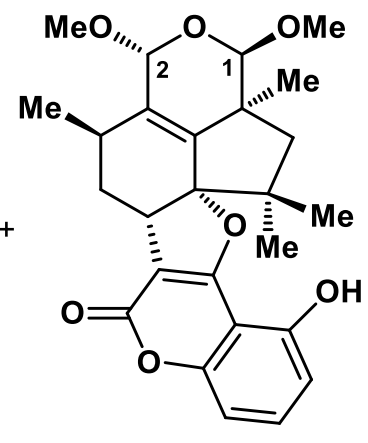

$C_{1,2}$-epi-hypocrolide A (22)

Compound 21 (300 mg, 0.57 mmol, 1.0 equiv.) was dissolved in dichloromethane (150 mL) and trifluoroacetic acid $(0.42 \mathrm{ml}, 5.70 \mathrm{mmol}, 10.0$ equiv. $)$ was added at $0^{\circ} \mathrm{C}$, followed by stirring at 25 ${ }^{\circ} \mathrm{C}$ for $12 \mathrm{~h}$. After completion of the reaction, the organic solution was concentrated under reduced pressure and methanol $(50 \mathrm{~mL})$ was added, followed by stirring for another $12 \mathrm{~h}$ at $25{ }^{\circ} \mathrm{C}$. The organic solution was concentrated under reduced pressure. The crude product was purified by silica gel column chromatography (hexane/ethyl acetate $=5 / 1)$ to give (-)-hypocrolide A $(6)(80$ $\mathrm{mg}, 31 \%$ yield) as a white solid and (-)- $\mathrm{C}_{1,2}$-epi-hypocrolide A (22) (77 mg, 30\% yield) as a white solid.

\section{Compound 6:}

$[\boldsymbol{\alpha}]_{\mathbf{D}}^{24}=-71.5\left(\mathrm{c}=0.50\right.$ in $\left.\mathrm{CHCl}_{3}\right),\left[\right.$ lit. $\left\{[\boldsymbol{\alpha}]_{\mathbf{D}}^{16.7}=-61(\mathrm{c}=0.1\right.$ in $\left.\left.\mathrm{MeOH})\right\}\right] ;$

$\mathbf{R}_{f}=0.45$ (hexane/ethyl acetate $=3 / 1$ );

IR (film) $\lambda_{\max } 3487,3449,2924,1721,1628,1466,1350,1265,1018,794,748$;

${ }^{1}$ H NMR (400 MHz, $\left.\mathbf{C D C l}_{3}\right) \delta 7.42(\mathrm{t}, J=8.3 \mathrm{~Hz}, 1 \mathrm{H}), 7.21(\mathrm{~s}, 1 \mathrm{H}), 6.92(\mathrm{~d}, J=8.4 \mathrm{~Hz}, 1 \mathrm{H})$, $6.77(\mathrm{~d}, J=8.3 \mathrm{~Hz}, 1 \mathrm{H}), 5.07$ (s, 1H), $4.50(\mathrm{~s}, 1 \mathrm{H}), 3.56$ (s, 3H), 3.54 (s, 3H), 3.45 (dd, $J=9.4,5.0$ $\mathrm{Hz}, 1 \mathrm{H}), 2.42-2.34(\mathrm{~m}, 1 \mathrm{H}), 2.06-1.96(\mathrm{~m}, 1 \mathrm{H}), 1.95-1.82(\mathrm{~m}, 1 \mathrm{H}), 1.81(\mathrm{~d}, J=14.1 \mathrm{~Hz}, 1 \mathrm{H})$, $1.73(\mathrm{~d}, J=14.2 \mathrm{~Hz}, 1 \mathrm{H}), 1.28(\mathrm{~s}, 3 \mathrm{H}), 1.16(\mathrm{~d}, J=7.1 \mathrm{~Hz}, 3 \mathrm{H}), 1.04(\mathrm{~s}, 3 \mathrm{H}), 0.96(\mathrm{~s}, 3 \mathrm{H})$. 
${ }^{13}$ C NMR (100 MHz, $\left.\mathbf{C D C l}_{3}\right) \delta$ 164.6, 159.9, 155.5, 153.4, 138.9, 137.9, 133.5, 110.9, 108.9, 106.0, 105.6, 101.8, 101.2, 100.3, 56.9, 55.9, 47.6, 45.3, 41.7, 35.4, 33.4, 28.4, 27.1, 22.9, 21.3, 17.9.

HRMS (ESI) calcd for $\mathrm{C}_{26} \mathrm{H}_{30} \mathrm{NaO}_{7}[\mathrm{M}+\mathrm{Na}]^{+}$Exact Mass: 477.1884; found: 477.1882.

Table S6 Compared NMR data $\left[\mathrm{CDC}_{3}\right]$ between our synthetic hypocrolide A and the isolated natural product.

\begin{tabular}{|c|c|c|c|c|c|c|}
\hline \multirow[b]{2}{*}{ Carbon } & \multicolumn{2}{|c|}{${ }^{1} H \& p p m(J)$} & \multirow[b]{2}{*}{$\begin{array}{c}\text { error } \\
\text { (iso. - syn.) }\end{array}$} & \multicolumn{3}{|c|}{${ }^{13} \mathrm{C} \& \mathrm{ppm}$} \\
\hline & $\begin{array}{l}\text { isolated } \\
(600 \mathrm{M})\end{array}$ & $\begin{array}{c}\text { synthesized } \\
(400 \mathrm{M})\end{array}$ & & $\begin{array}{r}\text { isolated } \\
(150 \mathrm{M})\end{array}$ & $\begin{array}{c}\text { synthesized } \\
(100 \mathrm{M})\end{array}$ & $\begin{array}{c}\text { error } \\
\text { (iso. - syn.) }\end{array}$ \\
\hline 1 & $4.50(\mathrm{~s})$ & $4.50(\mathrm{~s}, 1 \mathrm{H})$ & 0 & 101.9 & 101.8 & 0.1 \\
\hline 2 & $5.07(\mathrm{~s})$ & $5.07(\mathrm{~s}, 1 \mathrm{H})$ & 0 & 100.4 & 100.3 & 0.1 \\
\hline 3 & & & & 137.7 & 137.9 & -0.2 \\
\hline 4 & $2.38(\mathrm{~m})$ & $2.42-2.34(\mathrm{~m}, 1 \mathrm{H})$ & 0 & 28.4 & 28.4 & 0 \\
\hline \multirow[t]{2}{*}{5} & $2.00(\mathrm{~m})$ & $2.06-1.96(\mathrm{~m}, 1 \mathrm{H})$ & 0 & 35.4 & 35.4 & 0 \\
\hline & $1.89(\mathrm{~m})$ & $1.95-1.82(\mathrm{~m}, 1 \mathrm{H})$ & 0 & & & \\
\hline 6 & 3.45 (dd, J = 9.42, $4.98 \mathrm{~Hz})$ & $3.45(\mathrm{dd}, \mathrm{J}=9.4,5.0 \mathrm{~Hz}, 1 \mathrm{H})$ & 0 & 33.4 & 33.4 & 0 \\
\hline 7 & & & & 106.1 & 106.0 & 0.1 \\
\hline 8 & & & & 45.3 & 45.3 & 0 \\
\hline 9 & $1.81(\mathrm{~d}, \mathrm{~J}=14.2 \mathrm{~Hz})$ & $1.81(\mathrm{~d}, \mathrm{~J}=14.1 \mathrm{~Hz}, 1 \mathrm{H})$ & 0 & 47.7 & 47.6 & 0.1 \\
\hline & $1.74(\mathrm{~d}, \mathrm{~J}=14.2 \mathrm{~Hz})$ & $1.73(\mathrm{~d}, \mathrm{~J}=14.2 \mathrm{~Hz}, 1 \mathrm{H})$ & 0.01 & & & \\
\hline 10 & & & & 41.7 & 41.7 & 0 \\
\hline 11 & & & & 138.8 & 138.9 & -0.1 \\
\hline 12 & & & & 105.7 & 105.6 & 0.1 \\
\hline 13 & & & & 164.7 & 164.6 & 0.1 \\
\hline 14 & & & & 155.5 & 155.5 & 0 \\
\hline 15 & $6.92(\mathrm{dd}, \mathrm{J}=8.34,0.78 \mathrm{~Hz})$ & $6.92(\mathrm{~d}, \mathrm{~J}=8.4 \mathrm{~Hz}, 1 \mathrm{H})$ & 0 & 108.9 & 108.9 & 0 \\
\hline 16 & $7.42(\mathrm{t}, \mathrm{J}=8.34 \mathrm{~Hz})$ & $7.42(\mathrm{t}, \mathrm{J}=8.3 \mathrm{~Hz}, 1 \mathrm{H})$ & 0 & 133.5 & 133.5 & 0 \\
\hline 17 & $6.76(d d, J=8.34,0.78 \mathrm{~Hz})$ & $6.77(\mathrm{~d}, \mathrm{~J}=8.3 \mathrm{~Hz}, 1 \mathrm{H})$ & -0.01 & 110.9 & 110.9 & 0 \\
\hline 18 & & & & 153.4 & 153.4 & 0 \\
\hline 19 & & & & 101.3 & 101.2 & 0.1 \\
\hline 20 & & & & 160.0 & 160.0 & 0 \\
\hline 21 & $3.56(\mathrm{~s})$ & $3.56(\mathrm{~s}, 3 \mathrm{H})$ & 0 & 57.0 & 56.9 & 0.1 \\
\hline 22 & $3.54(\mathrm{~s})$ & $3.54(\mathrm{~s}, 3 \mathrm{H})$ & 0 & 55.9 & 55.9 & 0 \\
\hline 23 & $1.17(\mathrm{~d} \mathrm{~J}=6.48 \mathrm{~Hz})$ & $1.16(\mathrm{~d}, \mathrm{~J}=7.1 \mathrm{~Hz}, 3 \mathrm{H})$ & 0.01 & 17.9 & 17.9 & 0 \\
\hline 24 & 0.96 (s) & $0.96(\mathrm{~s}, 3 \mathrm{H})$ & 0 & 27.1 & 27.0 & 0.1 \\
\hline 25 & $1.04(\mathrm{~s})$ & $1.04(\mathrm{~s}, 3 \mathrm{H})$ & 0 & 22.9 & 22.9 & 0 \\
\hline 26 & $1.29(\mathrm{~s})$ & $1.28(\mathrm{~s}, 3 \mathrm{H})$ & 0.01 & 21.3 & 21.3 & 0 \\
\hline $\mathrm{OH}-18$ & 7.20 (s) & $7.21(\mathrm{~s}, 1 \mathrm{H})$ & -0.01 & & & \\
\hline
\end{tabular}

\section{Compound 22:}

$\mathbf{R}_{f}=0.46$ (hexane/ethyl acetate $=3 / 1$ );

$[\boldsymbol{\alpha}]_{\mathbf{D}}^{25}=-96.8\left(\mathrm{c}=0.50\right.$ in $\left.\mathrm{CHCl}_{3}\right) ;$

IR (film) $\lambda_{\max } 3500,2993,2361,1767,1628,1466,1373,1242,1049,756$;

${ }^{1} \mathbf{H}$ NMR $\left(400 \mathrm{MHz}, \mathbf{C D C l}_{3}\right) \delta 7.41(\mathrm{t}, J=8.3 \mathrm{~Hz}, 1 \mathrm{H}), 7.21(\mathrm{~s}, 1 \mathrm{H}), 6.91(\mathrm{~d}, J=8.3 \mathrm{~Hz}, 1 \mathrm{H})$, $6.75(\mathrm{~d}, J=8.3 \mathrm{~Hz}, 1 \mathrm{H}), 5.20(\mathrm{~s}, 1 \mathrm{H}), 4.63(\mathrm{~s}, 1 \mathrm{H}), 3.48(\mathrm{dd}, J=8.3,5.2 \mathrm{~Hz}, 1 \mathrm{H}), 3.45(\mathrm{~s}, 3 \mathrm{H})$, $3.43(\mathrm{~s}, 3 \mathrm{H}), 2.46-2.50(\mathrm{~m}, 1 \mathrm{H}), 2.08-1.99(\mathrm{~m}, 2 \mathrm{H}), 1.97-1.88(\mathrm{~m}, 1 \mathrm{H}), 1.63(\mathrm{~d}, J=13.4 \mathrm{~Hz}$, 1H), $1.43(\mathrm{~s}, 3 \mathrm{H}), 1.08(\mathrm{~d}, J=7.1 \mathrm{~Hz}, 3 \mathrm{H}), 1.06(\mathrm{~s}, 3 \mathrm{H}), 0.98(\mathrm{~s}, 3 \mathrm{H})$; 
${ }^{13}$ C NMR (100 MHz, $\left.\mathbf{C D C l}_{3}\right) \delta$ 164.5, 159.9, 155.3, 153.3, 137.3, 135.7, 133.3, 110.7, 108.7, 106.4, 106.1, 105.2, 101.3, 94.0, 55.7, 54.3, 47.5, 44.1, 41.1, 34.0, 33.6, 29.3, 26.2, 25.2, 23.2, 17.1

HRMS (ESI) calcd for $\mathrm{C}_{26} \mathrm{H}_{30} \mathrm{NaO}_{7}[\mathrm{M}+\mathrm{Na}]^{+}$Exact Mass: 477.1884; found: 477.1882.

Synthesis of (-)-hypocrolide A (6) from $\mathrm{C}_{1,2^{-}}$epi-hypocrolide A (22)

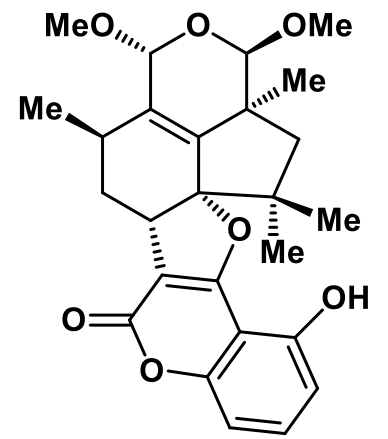

$\mathrm{C}_{1,2}$-epi-hypocrolide A (22)
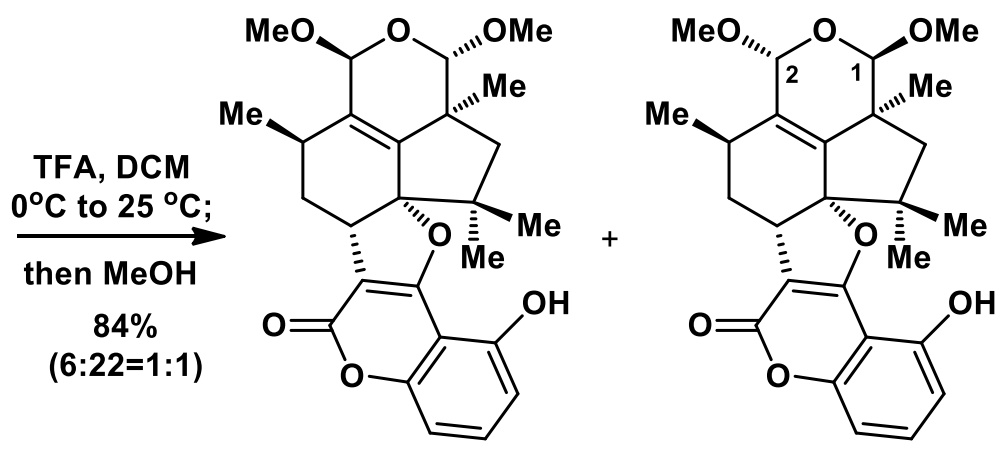

(-)-hypocrolide A (6) $\quad C_{1,2}$-epi-hypocrolide A (22)

Compound 22 (77 mg, $0.17 \mathrm{mmol}, 1.0$ equiv.) was dissolved in dichloromethane (100 mL) and trifluoroacetic acid $(0.12 \mathrm{ml}, 1.70 \mathrm{mmol}, 10.0$ equiv. $)$ was added at $0^{\circ} \mathrm{C}$, followed by stirring at 25 ${ }^{\circ} \mathrm{C}$ for 10 min. After completion of the reaction, the organic solution was concentrated under reduced pressure and methanol $(30 \mathrm{~mL})$ was added, followed by stirring for $12 \mathrm{~h}$ at $25{ }^{\circ} \mathrm{C}$. The organic solution was concentrated under reduced pressure. The crude product was purified by silica gel column chromatography (hexane/ethyl acetate $=5 / 1)$ to give $(-)$-hypocrolide $(2)(32$ mg, $42 \%$ yield, $84 \%$ brsm yield) as a white solid and (-)- $\mathrm{C}_{1,2^{-}}$epi-hypocrolide A (22) (32 mg, $42 \%$ yield) as a white solid recovered.

Note: Using the route described above, a total of more than $110 \mathrm{mg}$ of (-)-hypocrolide A was prepared readily. 
Racemic synthesis of ( \pm )-hypocrolide A for HPLC analysis<smiles>CC1(C)C=CC(=O)CC1</smiles>

13

( \pm )-hypocrolide A<smiles>[Y4]C1(C)CC2(C)[C@@H](OC)OC(OC)C3=C4C(C)C[C@H]2C2=C3O[C@@]41c1c(O)cccc1OC2=O</smiles>

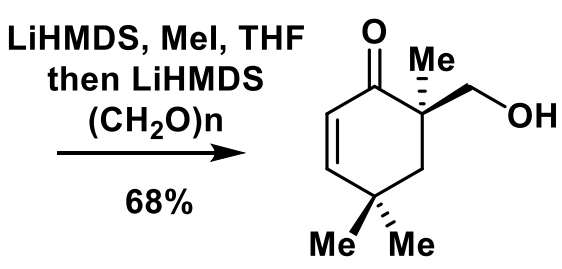

$( \pm)-13 a$

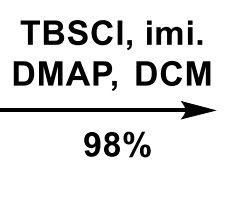

$( \pm)-13 b$<smiles>CCCCOCC1(C)CC(C)(C)C=CC1=O</smiles>

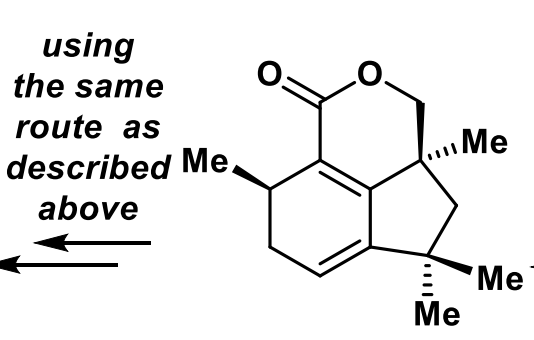

$( \pm)$-10-oxodihydrobotry-1(9)4(5)-diendial

(2)

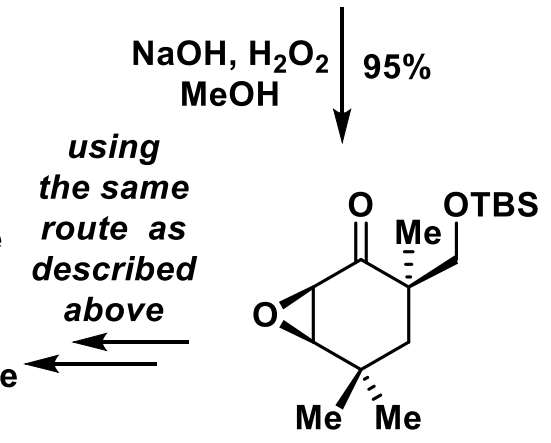

$( \pm)-11$

Synthesis of $( \pm)-13 a$<smiles>CC1(C)C=CC(=O)CC1</smiles>

13
LiHMDS

Mel, THF

then LiHMDS

$\left(\mathrm{CH}_{2} \mathrm{O}\right) \mathrm{n}$

$68 \%$<smiles>CC1(C)C=CC(=O)[C@](C)(CO)C1</smiles>

$( \pm)-13 a$

A solution of 4,4-dimethyl-cyclohex-2-en-1-one $\mathbf{1 3}$ (10.0 g, $80.6 \mathrm{mmol}, 1$ equiv) in tetrahydrofuran $(130 \mathrm{~mL})$ was added to a solution of LHMDS (1 M in tetrahydrofuran, $104.8 \mathrm{~mL}$, $104.8 \mathrm{mmol}, 1.3$ equiv) in tetrahydrofuran $(150 \mathrm{~mL})$ at $-78{ }^{\circ} \mathrm{C}$, and stirring for $0.5 \mathrm{~h}$. Then the reaction device was transferred into an ice-water bath and the reaction mixture was stirred for another $0.5 \mathrm{~h}$. Iodomethane $(7.5 \mathrm{~mL}, 120.9 \mathrm{mmol}, 1.5$ equiv) was added dropwise to the flask, then the mixture was allowed to warm to $25^{\circ} \mathrm{C}$ in $1 \mathrm{~h}$. Recooling the mixture to $-78^{\circ} \mathrm{C}$, then it was added another solution of LHMDS (1 $\mathrm{M}$ in tetrahydrofuran, $120.9 \mathrm{~mL}, 120.9 \mathrm{mmol}, 1.5$ equiv) and stirring for $0.5 \mathrm{~h}$. Then the reaction device was transferred into an ice-water bath and the reaction mixture was stirred for another $0.5 \mathrm{~h}$. Paraformaldehyde (14.5 g, $161.2 \mathrm{mmol}, 2.0$ equiv) 
was added to the flask, then the mixture was allowed to stir for another $0.5 \mathrm{~h}$. A saturated aqueous solution of $\mathrm{NH}_{4} \mathrm{Cl}(100 \mathrm{~mL})$ was added and the mixture was extracted with ethyl acetate $(3 \times 200$ $\mathrm{mL}$ ). The combined organic phases were dried over anhydrous sodium sulfate, filtered and concentrated under reduced pressure. The crude product was purified by silica gel column chromatography (hexane/ethyl acetate $=10 / 1)$ to give $( \pm)-\mathbf{1 3 a}(9.2 \mathrm{~g}, 68 \%$ yield $)$ as a light yellow oil.

$\mathbf{R}_{f}=0.3$ (hexane/ethyl acetate $=2 / 1$ );

IR (film) $\lambda_{\max } 3460,2963,2358,1732,1456,1261,1094,1022,864,798$;

${ }^{1} \mathbf{H}$ NMR $\left(400 \mathrm{MHz}, \mathbf{C D C l}_{3}\right) \delta 6.62(\mathrm{dd}, J=10.1,1.7 \mathrm{~Hz}, 1 \mathrm{H}), 5.80(\mathrm{~d}, J=10.1 \mathrm{~Hz}, 1 \mathrm{H}), 3.44-3.56$ (m, 2H), $2.73(\mathrm{~s}, 1 \mathrm{H}), 1.97(\mathrm{~d}, J=14.2 \mathrm{~Hz}, 1 \mathrm{H}), 1.56(\mathrm{dd}, J=14.2,1.7 \mathrm{~Hz}, 1 \mathrm{H}), 1.27(\mathrm{~s}, 3 \mathrm{H}), 1.24(\mathrm{~s}$, $3 \mathrm{H}), 1.15(\mathrm{~s}, 3 \mathrm{H})$;

${ }^{13} \mathbf{C}$ NMR (100 MHz, $\left.\mathbf{C D C l}_{3}\right) \delta$ 205.7, 158.9, 125.1, 69.3, 45.4, 43.6, 33.0, 32.4, 29.0, 22.3;

HRMS (ESI) calcd for $\mathrm{C}_{10} \mathrm{H}_{16} \mathrm{NaO}_{2}[\mathrm{M}+\mathrm{Na}]^{+}$Exact Mass: 191.1042; found: 191.1043.

\section{Synthesis of $( \pm)-13 b$}

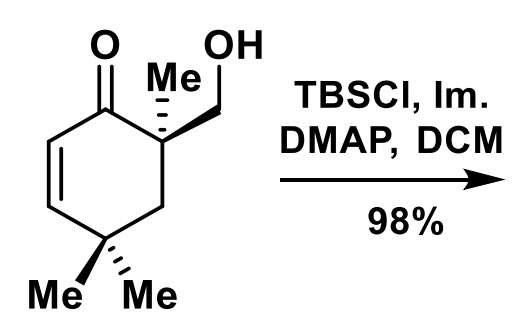

$( \pm)-13 a$

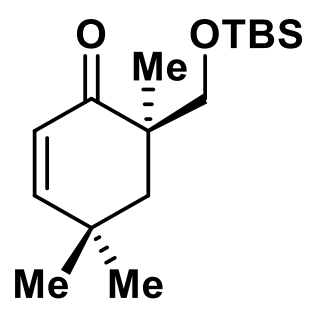

$( \pm)-13 b$

To a solution of 6-(hydroxymethyl)-4,4,6-trimethylcyclohex-2-enone ((士)-13a, $43.0 \mathrm{~g}, 233.6$ mmol, 1.0 equiv), imidazole (15.9 g, $233.6 \mathrm{mmol}, 1.0$ equiv) and catalyst amount 4-dimethylaminopyridine (1.4 g, $11.7 \mathrm{mmol}, 0.05$ equiv) in $\mathrm{CH}_{2} \mathrm{Cl}_{2}(300 \mathrm{~mL})$ was added TBSCl (42.2 g, $280.3 \mathrm{mmol}, 1.2$ equiv), the mixture was stirred at $25{ }^{\circ} \mathrm{C}$ for $12 \mathrm{~h}$. A saturated aqueous solution of $\mathrm{NH}_{4} \mathrm{Cl}(100 \mathrm{~mL})$ was added and the mixture was extracted with $\mathrm{CH}_{2} \mathrm{Cl}_{2}(3 \times 200 \mathrm{~mL})$. The combined organic phases were dried over anhydrous sodium sulfate, filtered and concentrated under reduced pressure. The crude product was purified by silica gel column chromatography (hexane/ethyl acetate $=50 / 1)$ to give $( \pm)-\mathbf{1 3 b}(68.0 \mathrm{~g}, 98 \%$ yield $)$ as a light yellow oil .

$\mathbf{R}_{f}=0.8($ hexane/ethyl acetate $=50 / 1)$

IR (film) $\lambda_{\max }$ 2959, 2928, 2857, 2359, 1678, 1472, 1361, 1260, 1096, 1026, 862, 837, 804; 
${ }^{1}$ H NMR $\left(400 \mathrm{MHz}, \mathbf{C D C l}_{3}\right) \delta 6.58(\mathrm{~d}, J=10.1 \mathrm{~Hz}, 1 \mathrm{H}), 5.80(\mathrm{~d}, J=10.1 \mathrm{~Hz}, 1 \mathrm{H}), 3.77(\mathrm{~d}, J=9.5 \mathrm{~Hz}$, $1 \mathrm{H}), 3.34(\mathrm{~d}, J=9.5 \mathrm{~Hz}, 1 \mathrm{H}), 2.20(\mathrm{~d}, J=14.2 \mathrm{~Hz}, 1 \mathrm{H}), 1.58(\mathrm{dd}, J=14.2,0.6 \mathrm{~Hz}, 1 \mathrm{H}), 1.19(\mathrm{~s}, 3 \mathrm{H})$, $1.17(\mathrm{~s}, 3 \mathrm{H}), 1.11(\mathrm{~s}, 3 \mathrm{H}), 0.86$ (s, 9H), $0.02(\mathrm{~s}, 3 \mathrm{H}), 0.01$ (s, 3H);

${ }^{13} \mathbf{C}$ NMR (100 MHz, $\left.\mathbf{C D C l}_{3}\right) \delta$ 203.0, 158.2, 125.8, 68.6, 46.6, 43.1, 32.8, 30.8, 30.4, 25.8, 22.8, 18.2, $-5.6,-5.6$

HRMS (ESI) calcd for $\mathrm{C}_{16} \mathrm{H}_{30} \mathrm{O}_{2} \mathrm{NaSi}[\mathrm{M}+\mathrm{Na}]^{+}$Exact Mass: 305.1907; found: 305.1905.

\section{Synthesis of $( \pm)-11$}

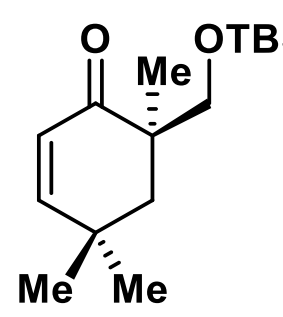

$( \pm)-13 b$

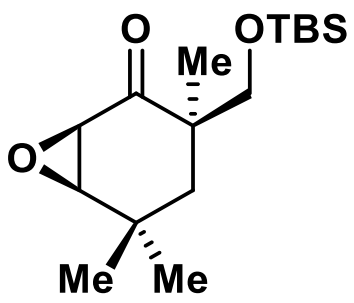

$( \pm)-11$

To a solution of 6-(((tert-butyldimethylsilyl)oxy)methyl)-4,4,6-trimethylcyclohex-2-enone $\left(( \pm)-\mathbf{1 3 b}, 45.0 \mathrm{~g}, 159.5 \mathrm{mmol}, 1.0\right.$ eq.) in methanol $(200 \mathrm{~mL})$ at $0{ }^{\circ} \mathrm{C}$ was added $30 \%$ aqueous $\mathrm{H}_{2} \mathrm{O}_{2}(53.2 \mathrm{~mL}, 3.0 \mathrm{eq})$ over a 10 minute period. This was followed by the dropwise addition of $2 \mathrm{~N} \mathrm{NaOH}(80.0 \mathrm{~mL}, 1.0 \mathrm{eq})$, the mixture was stirred at $0{ }^{\circ} \mathrm{C}$ for 5.0 hours. A saturated aqueous solution of $\mathrm{Na}_{2} \mathrm{SO}_{3}(100 \mathrm{~mL})$ was added and the mixture was extracted with $\mathrm{CH}_{2} \mathrm{Cl}_{2}(3 \times 200 \mathrm{~mL})$. The combined organic phases were dried over anhydrous sodium sulfate, filtered and concentrated under reduced pressure. The crude product was purified by silica gel column chromatography (hexane/ethyl acetate $=50 / 1)$ to give $( \pm)-\mathbf{1 1}(45.2 \mathrm{~g}, 95 \%$ yield $)$. 


\section{HPLC Analysis of $e e$ Value of Synthetic Hypocrolide A}

HPLC analysis: Daicel Chiralpak AD-H column; hexane $i$-propanol $=83: 17,1$ $\mathrm{mL} / \mathrm{min}, \lambda=230 \mathrm{~nm} ; t_{\mathrm{R}}$ (major) $=3.9 \mathrm{~min}, t_{\mathrm{R}}$ (major) $=5.2 \mathrm{~min} .>99 \%$ ee .
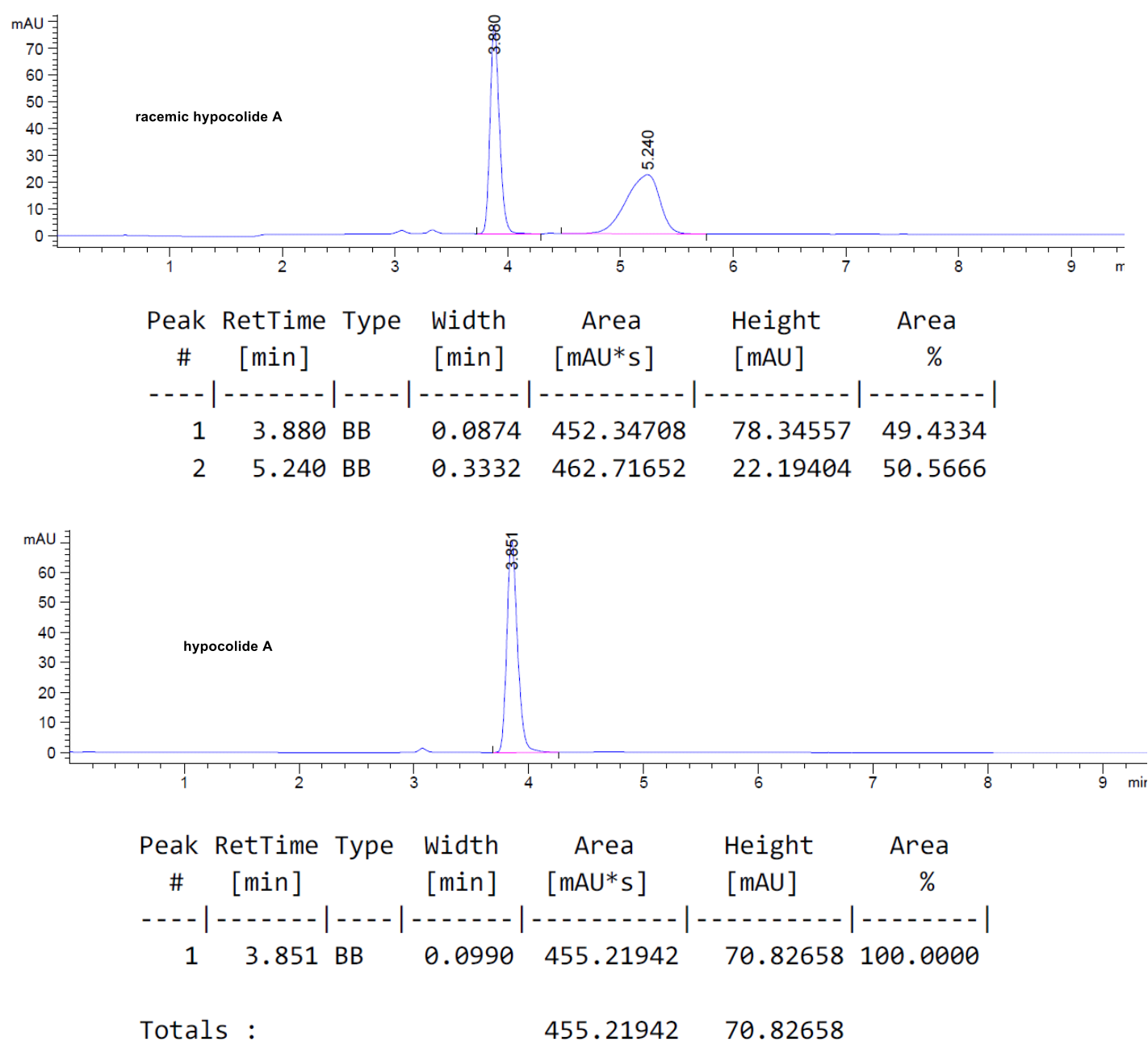


\section{X-ray crystal structures}

X-ray crystal structure of 11a (CCDC 1497665)

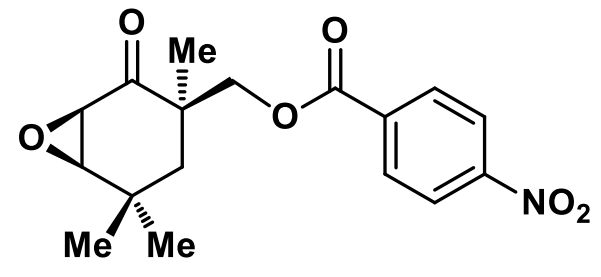

$11 \mathrm{a}$

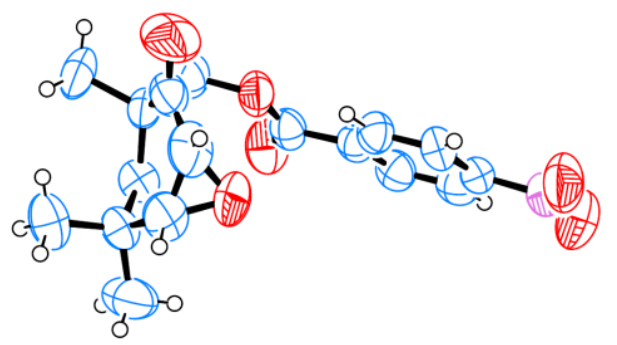

X-ray crystal structure of 8a (CCDC 1497643)

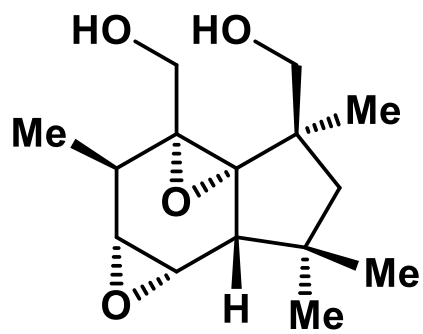

$8 \mathbf{a}$

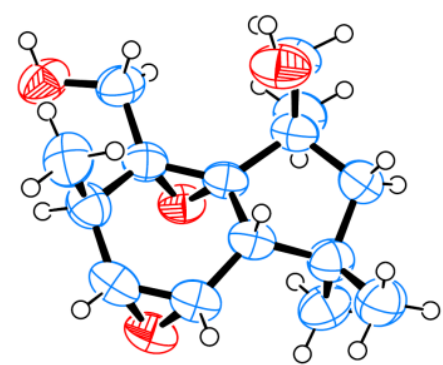

X-ray crystal structure of 16b (CCDC 1497666)<smiles>CC1C=C[C@]2(C)C(=C1COC(=O)c1ccc([N+](=O)[O-])cc1)[C@](C)(COC(=O)c1ccc([N+](=O)[O-])cc1)CC2(C)C</smiles>

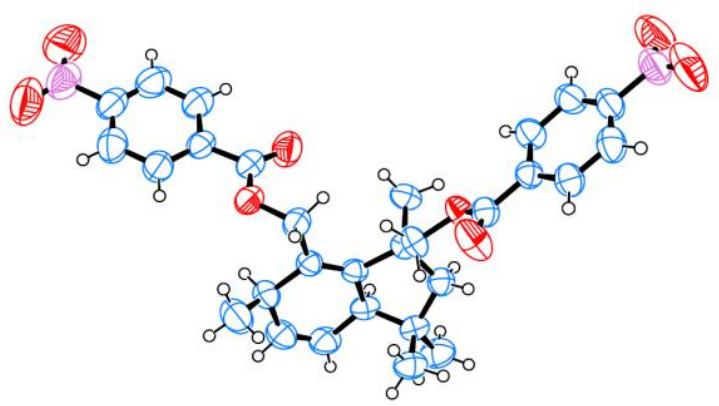

20 
X-ray crystal structure of 19a (CCDC 1497668)
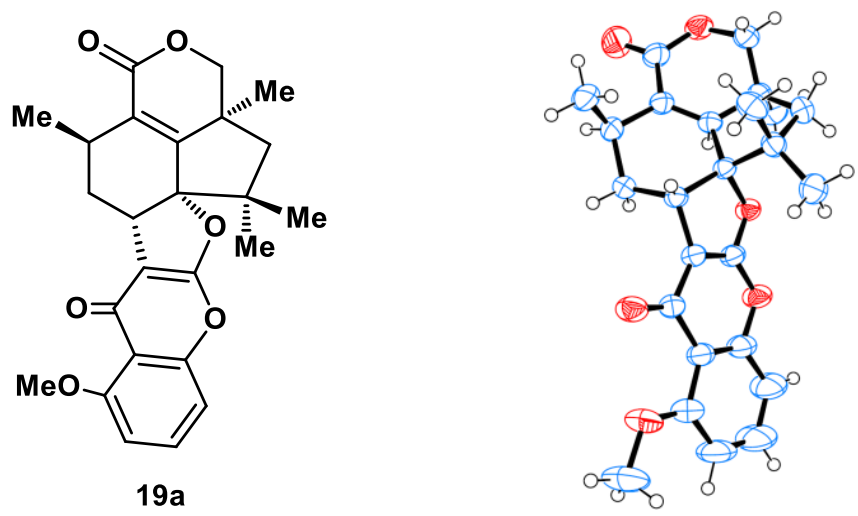

X-ray crystal structure of 19b (CCDC 1497669)

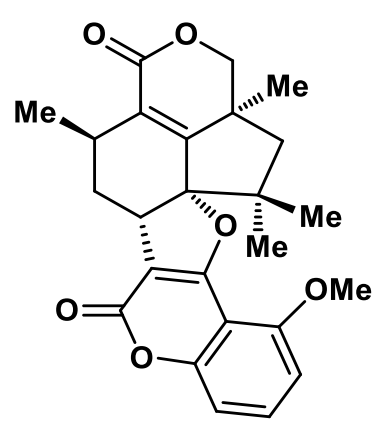

$19 b$

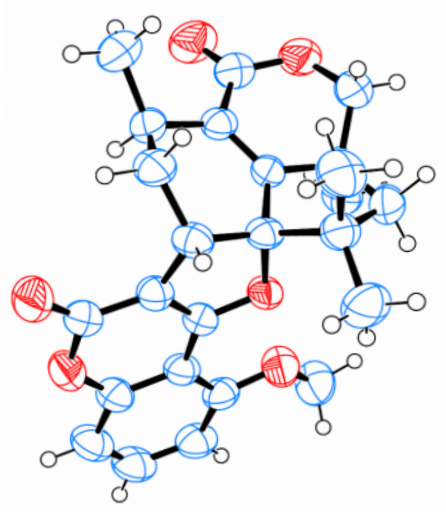


V. $\quad{ }^{1} \mathrm{H}$ and ${ }^{13} \mathrm{C}$ NMR Spectra
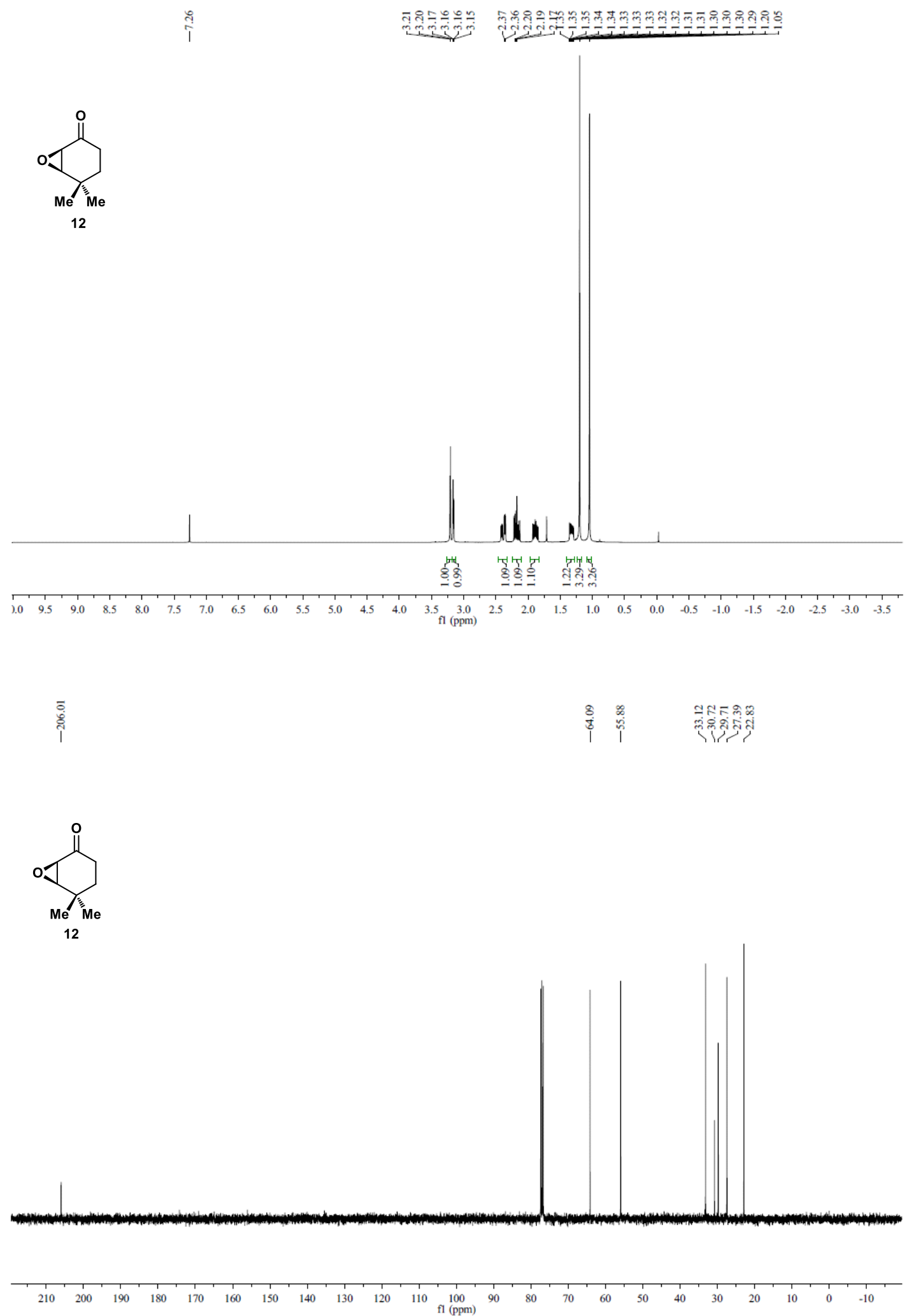

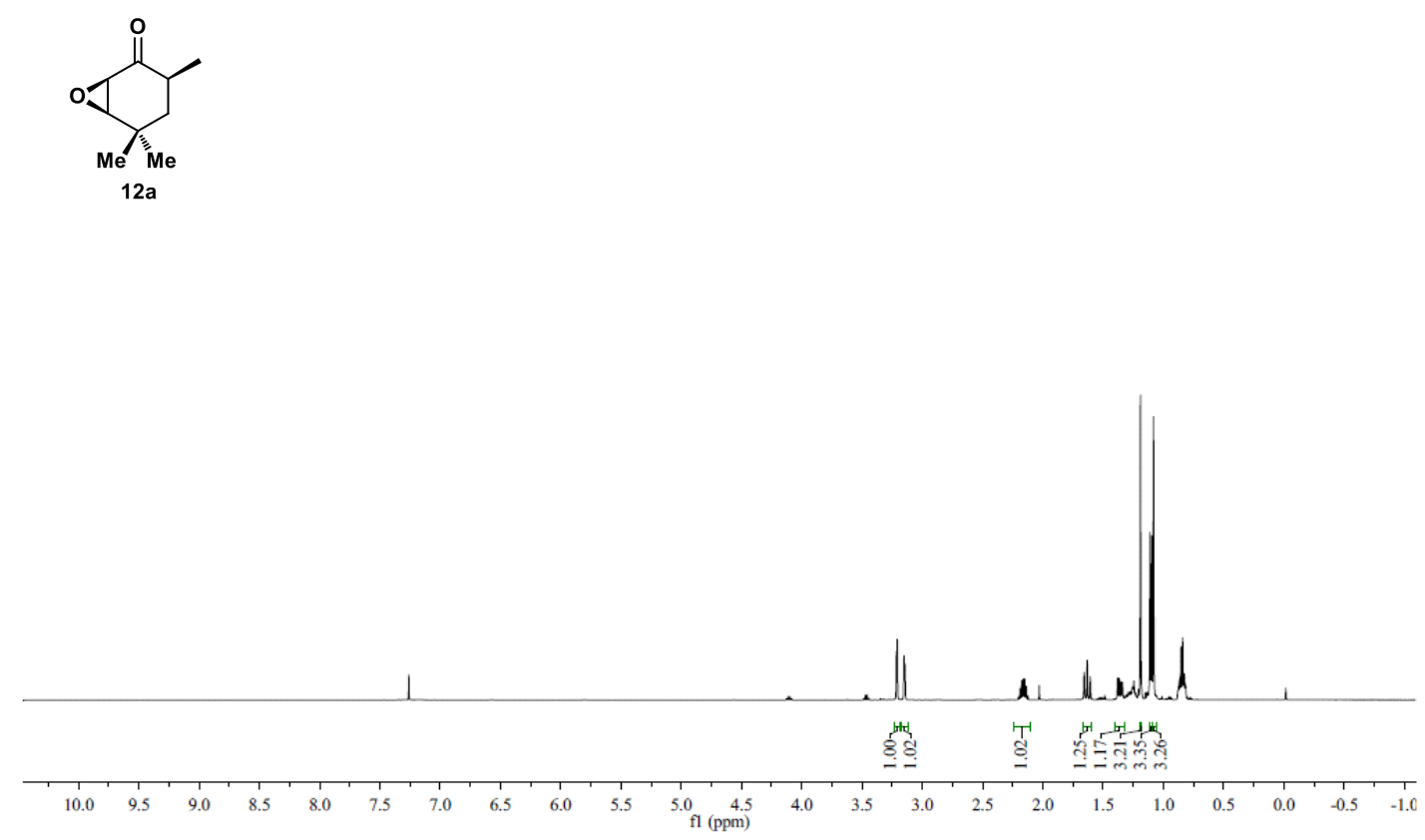

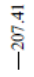

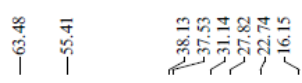
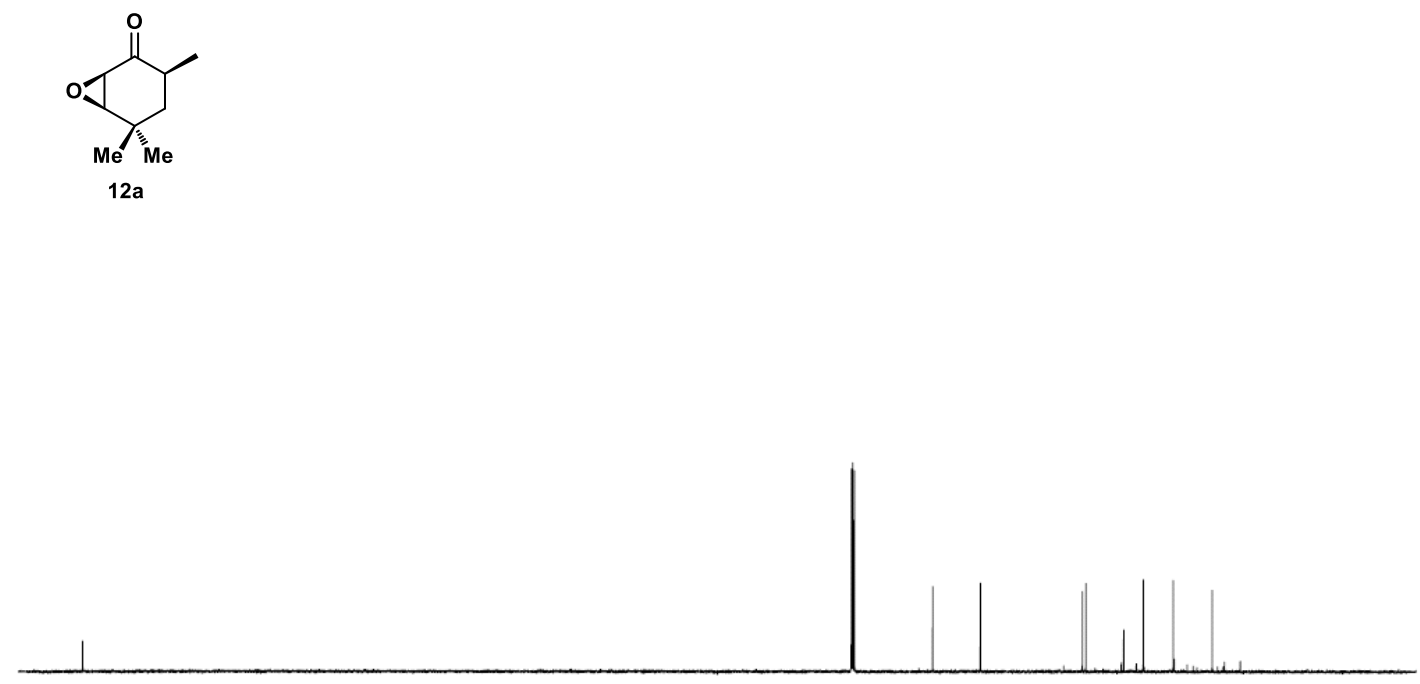

$\begin{array}{rlllllllllllllllllllllllll}1 & 1 \\ 210 & 200 & 190 & 180 & 170 & 160 & 150 & 140 & 130 & 120 & 110 & 100 & 90 & 80 & 70 & 60 & 50 & 40 & 30 & 20 & 10 & 0 & -10\end{array}$ 


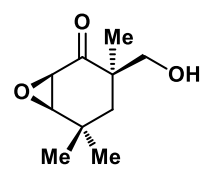

12b
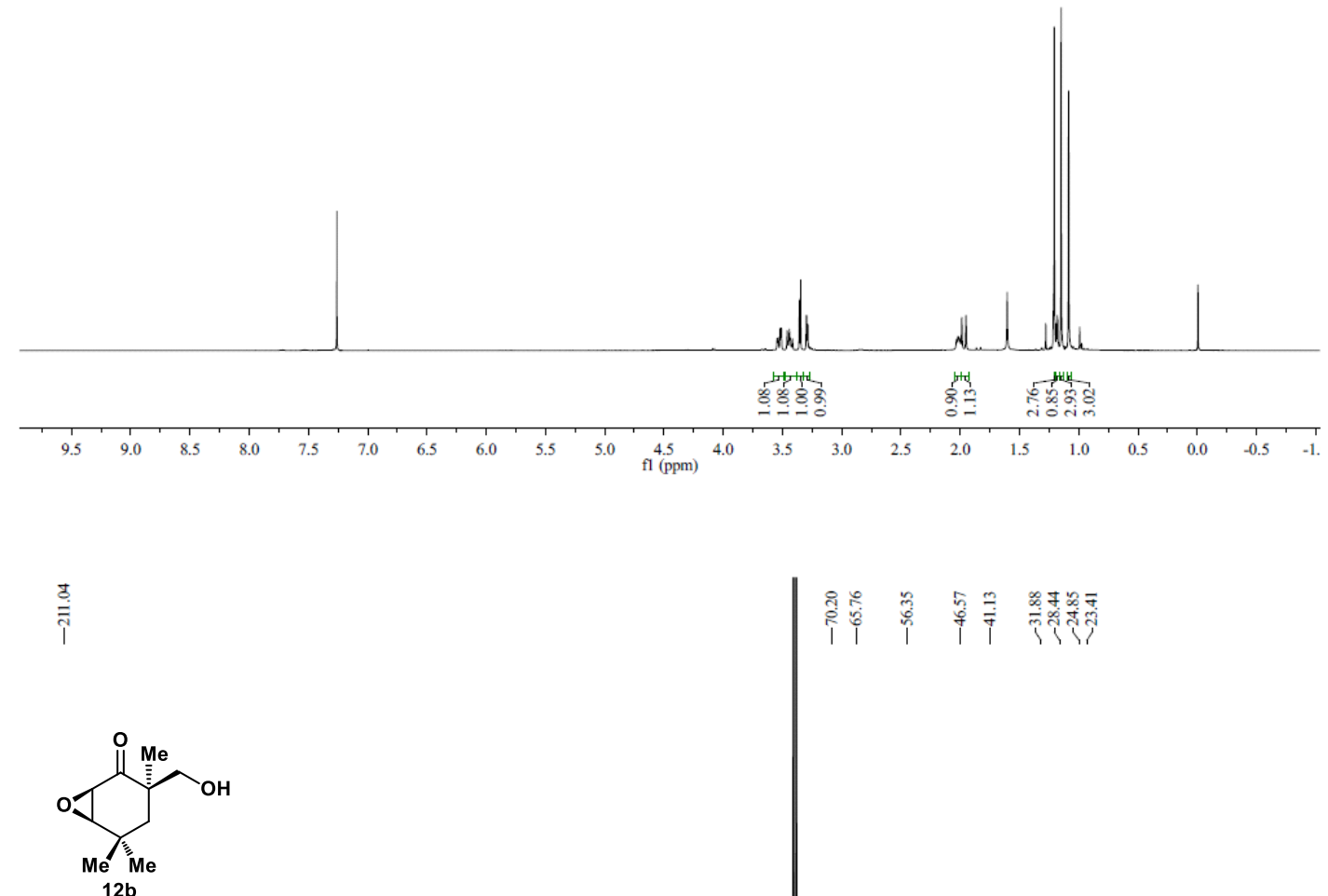

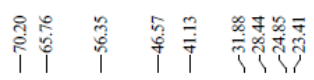

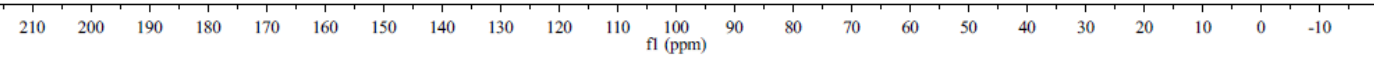



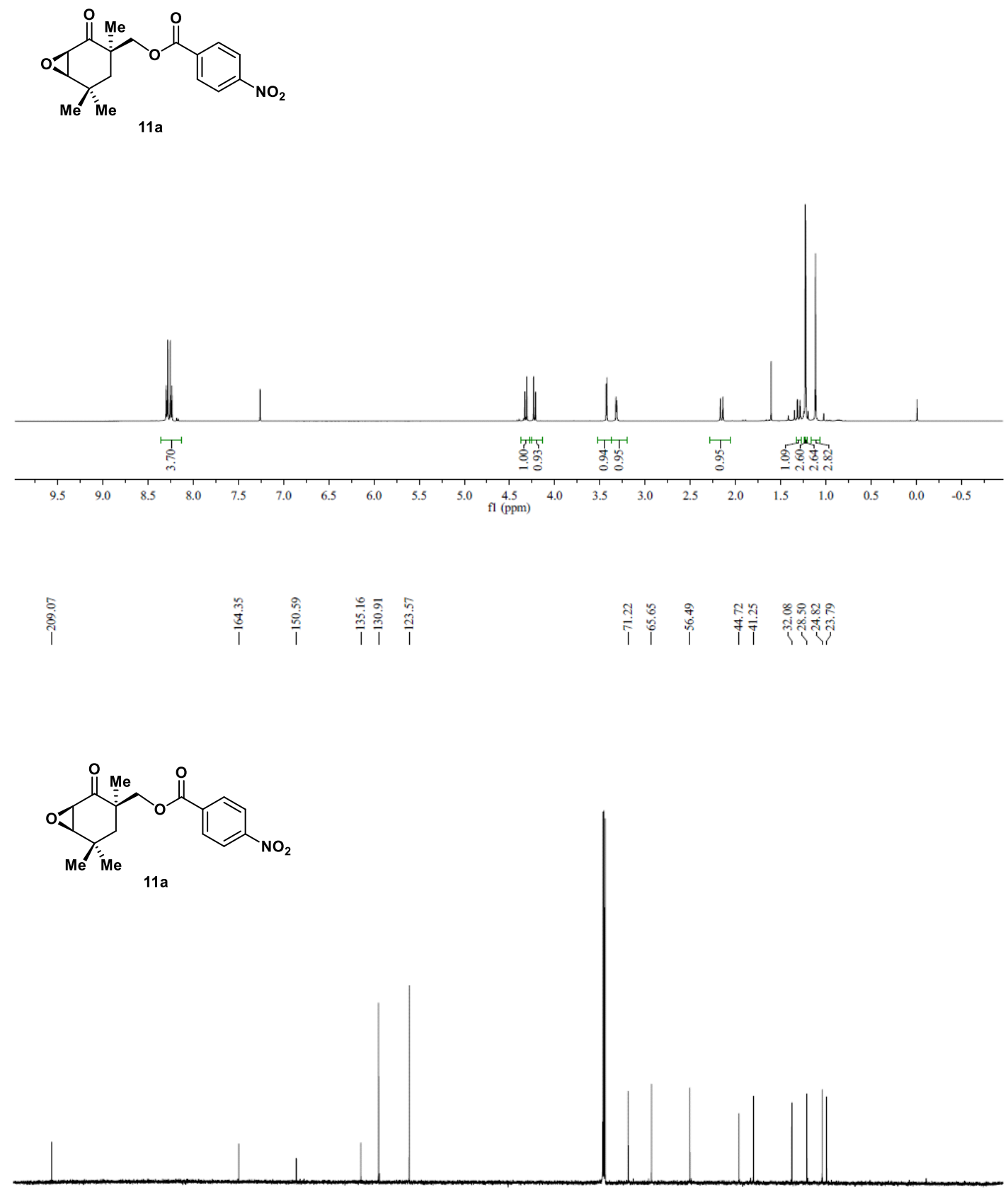

$\begin{array}{llllllllllllllllllllllllll}1 & 1 \\ 210 & 200 & 190 & 180 & 170 & 160 & 150 & 140 & 130 & 120 & 110 & \begin{array}{c}100 \\ \mathrm{fl}(\mathrm{ppm})\end{array} & 90 & 80 & 70 & 60 & 50 & 40 & 30 & 20 & 10 & 0 & -10\end{array}$ 


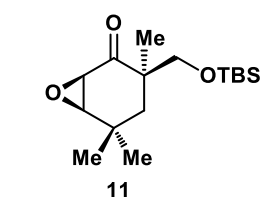

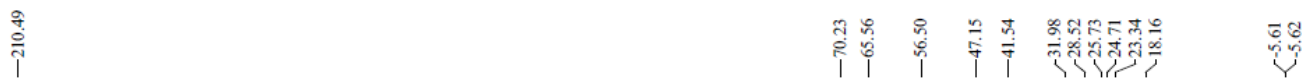

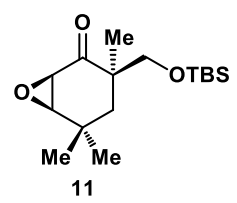

il he

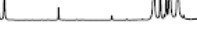

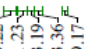
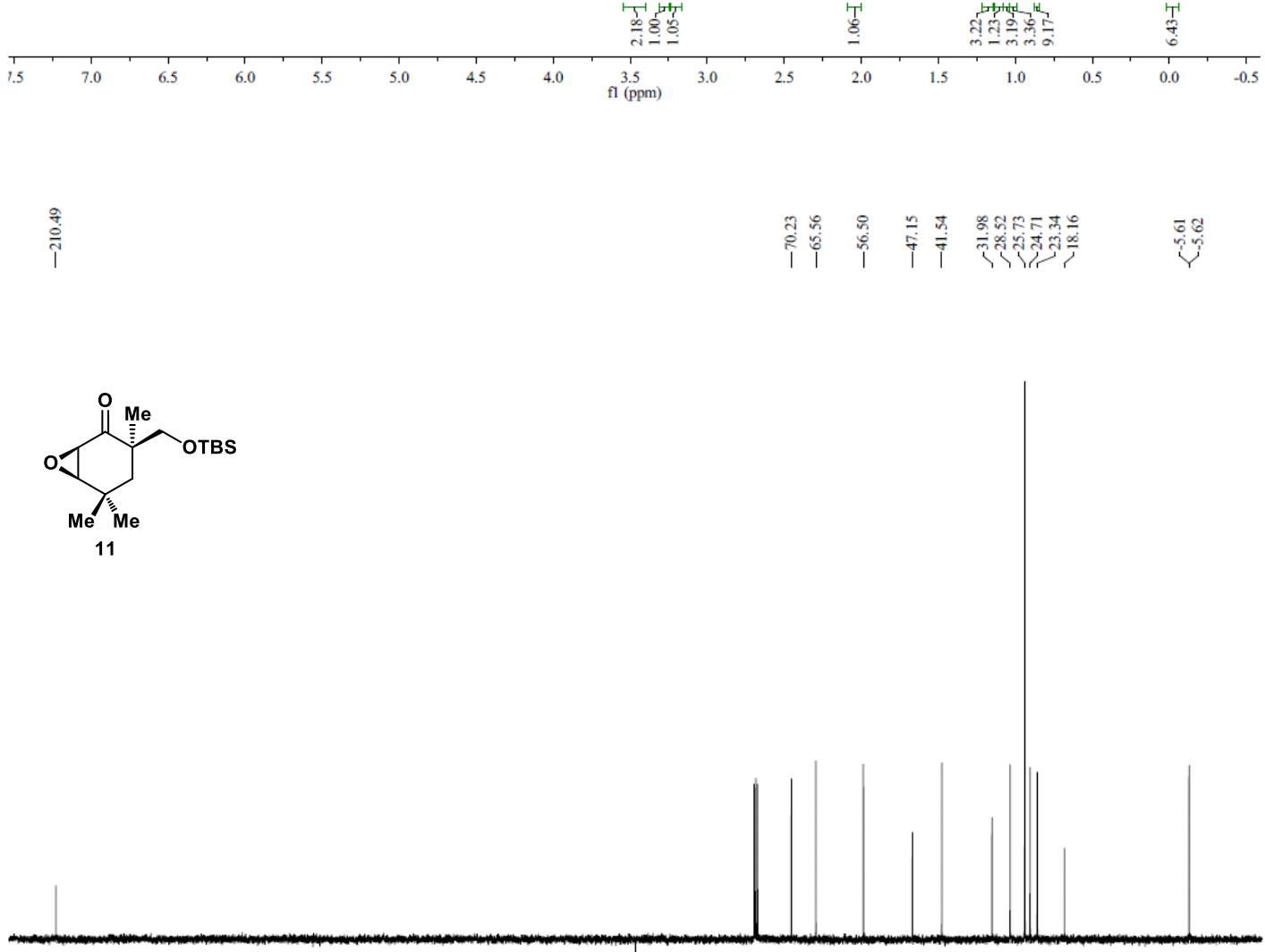

$\begin{array}{lllllllllllllllllllllllllllllll}210 & 200 & 190 & 180 & 170 & 160 & 150 & 140 & 130 & 120 & 110 & 100 & 90 & 80 & 70 & 60 & 50 & 40 & 30 & 20 & 10 & 0 & -10\end{array}$ 


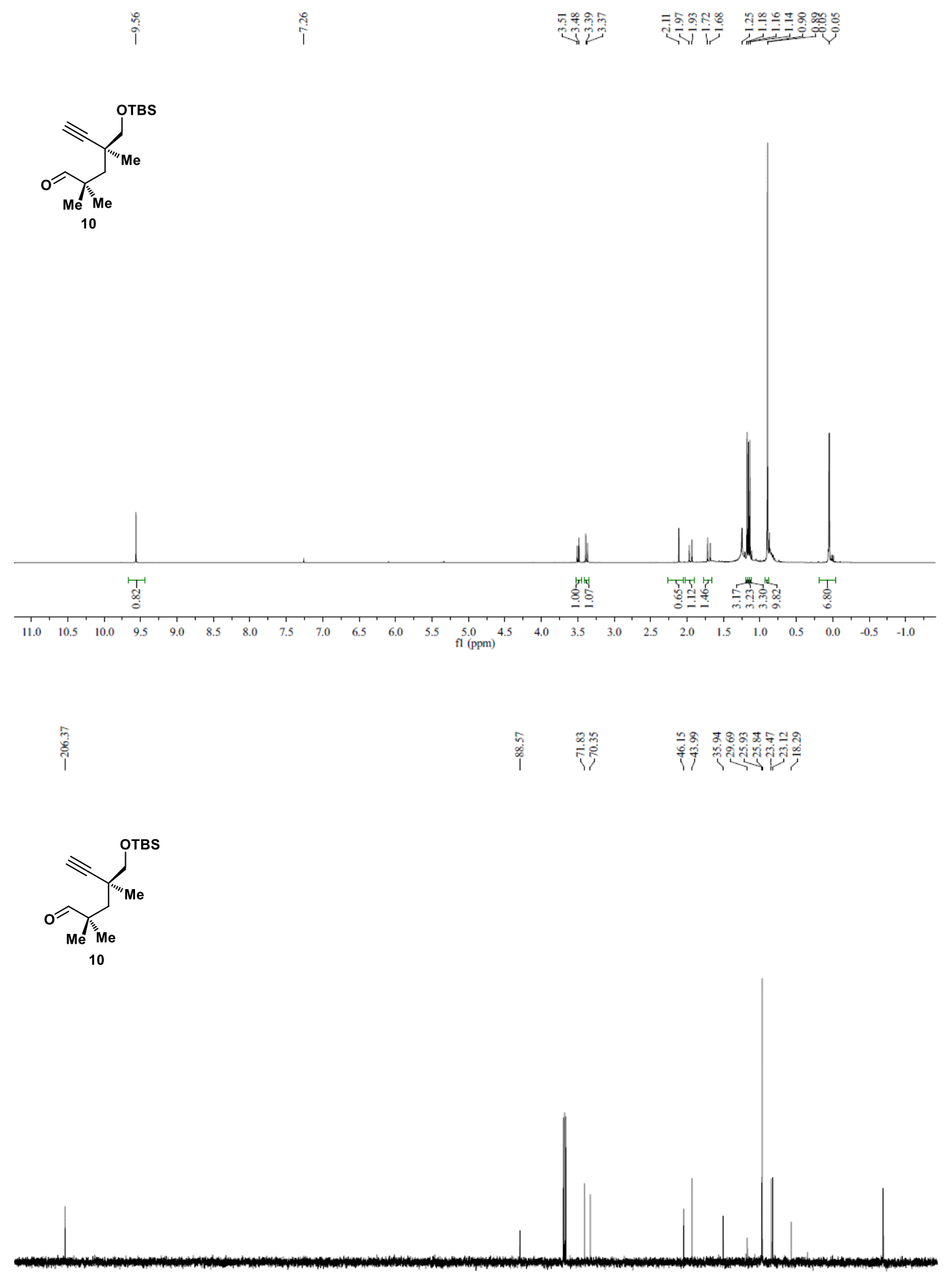

$\begin{array}{lllllllllllllllllllllllllllllll}1 & 1 \\ 210 & 200 & 190 & 180 & 170 & 160 & 150 & 140 & 130 & 120 & 110 & \begin{array}{c}100 \\ \mathrm{fl}(\mathrm{pm})\end{array} & 90 & 80 & 70 & 60 & 50 & 40 & 30 & 20 & 10 & 0 & -10\end{array}$ 

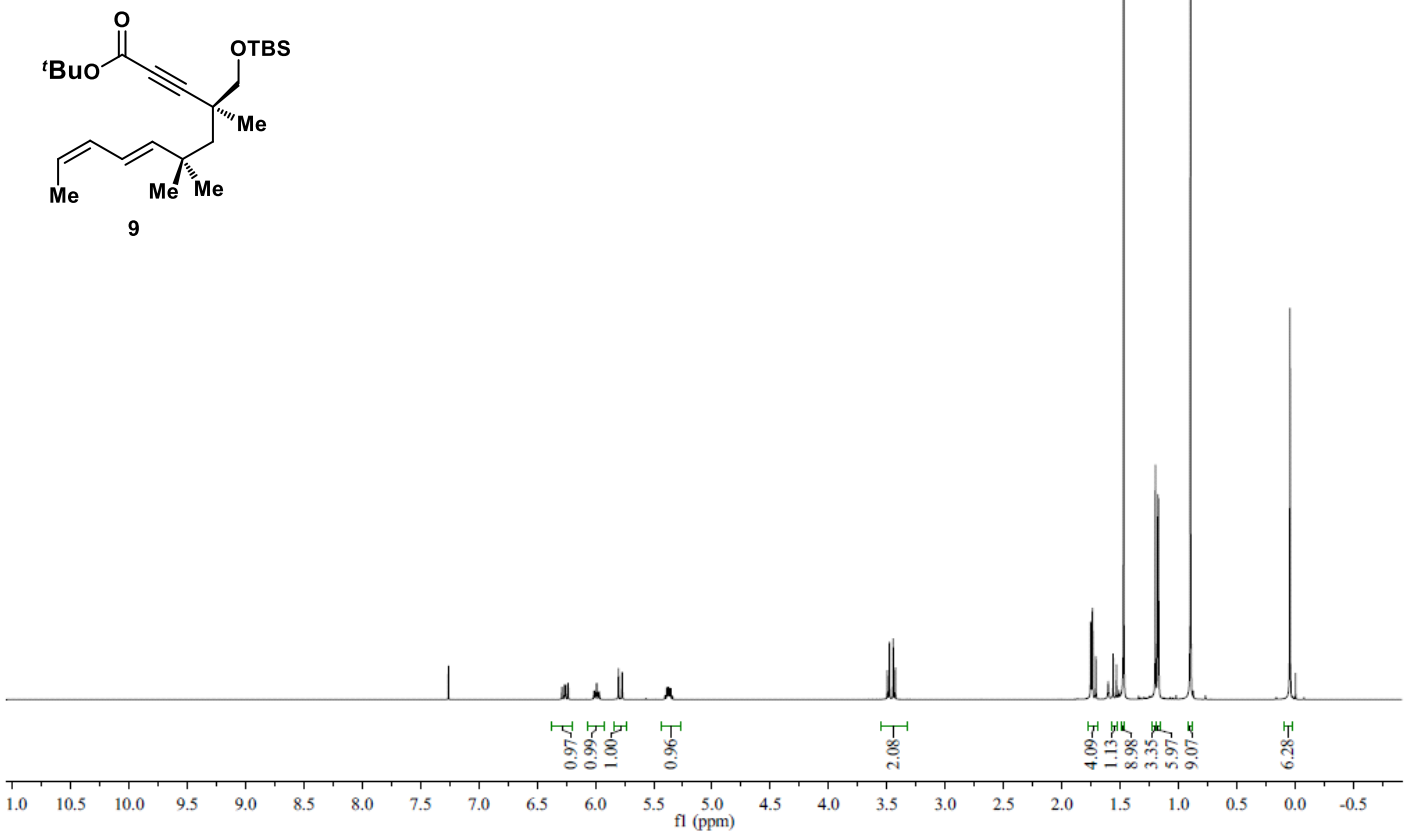<smiles>C/C=C\C=C/C(C)(C)CC(C)(C)CO</smiles> 


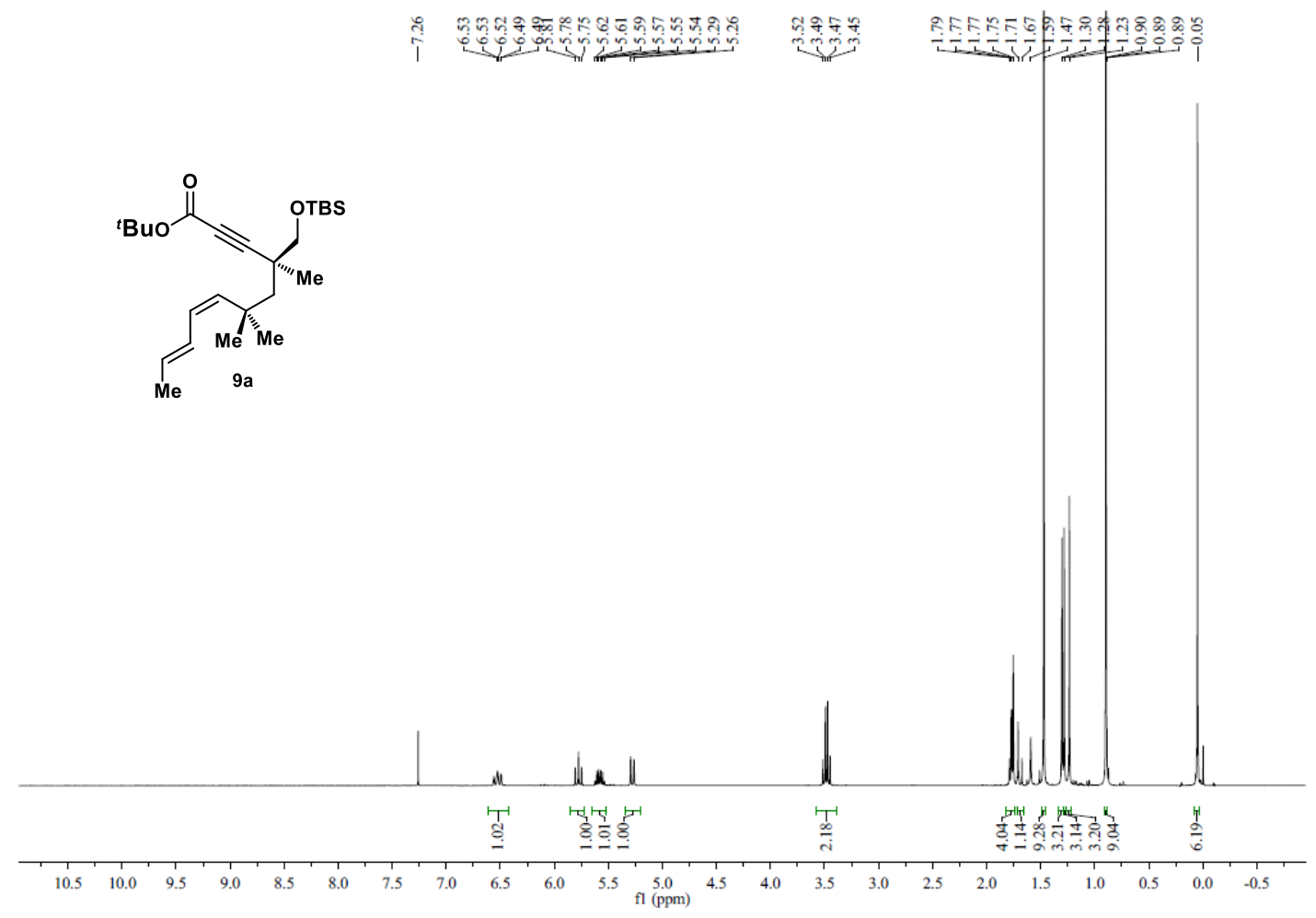

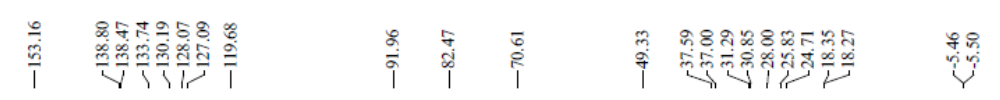

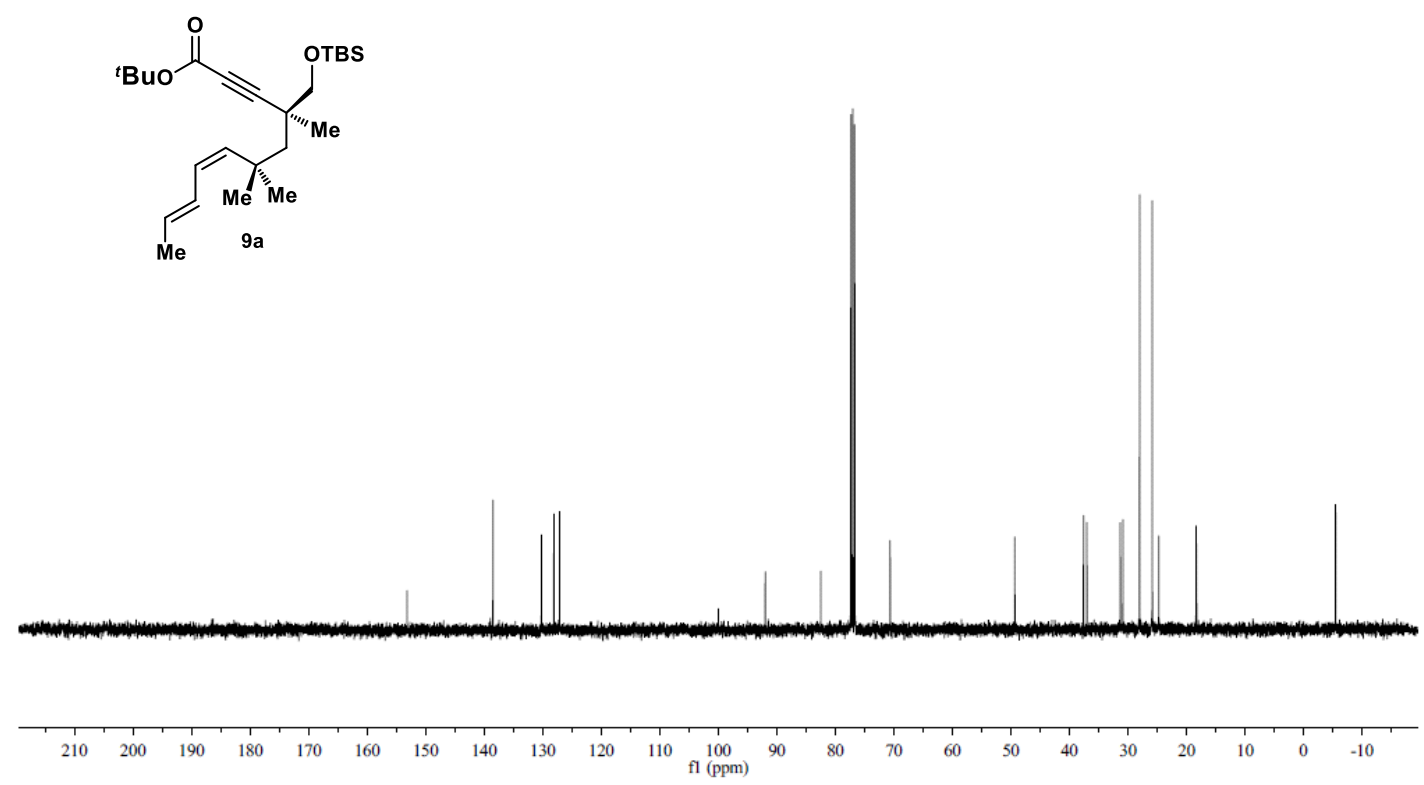



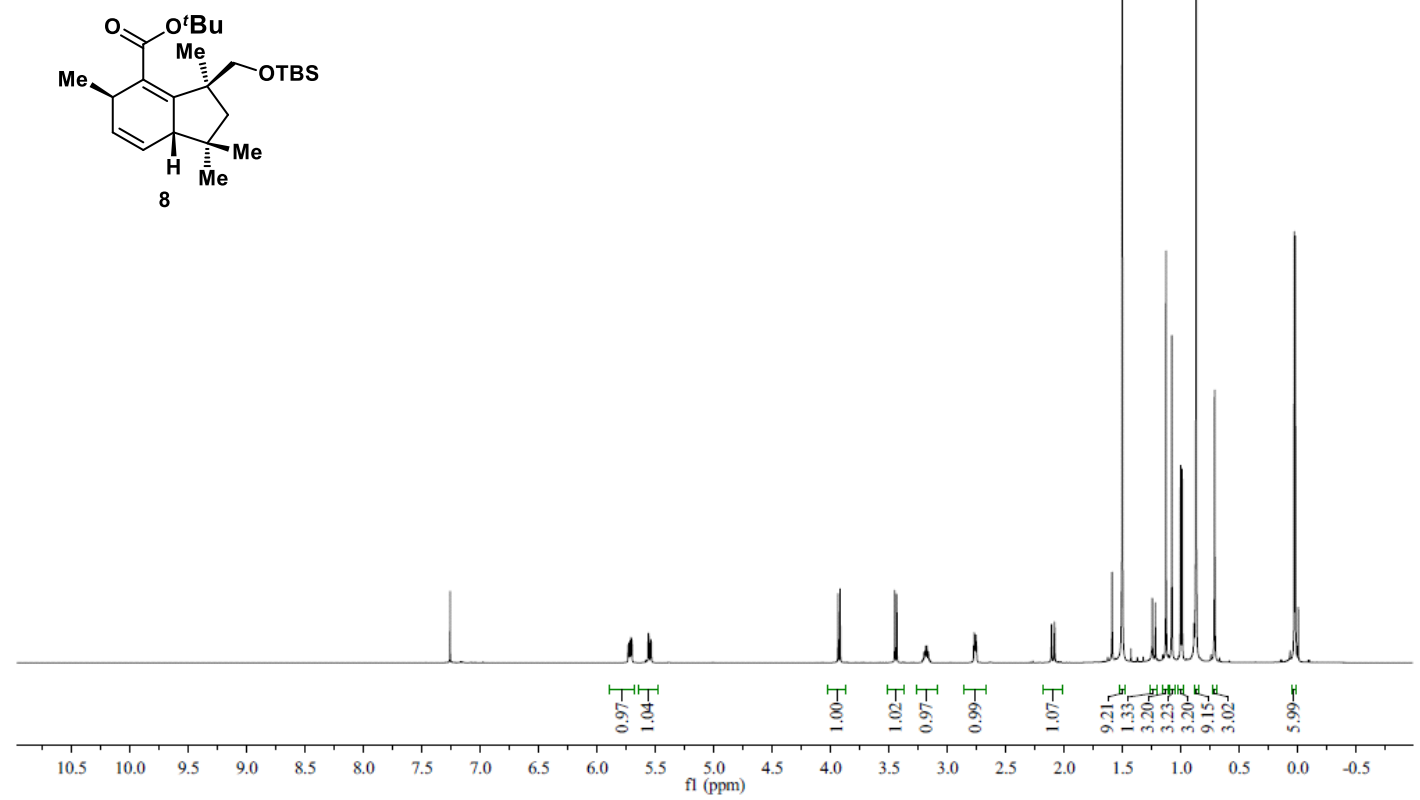

\begin{tabular}{|c|c|c|c|c|}
\hline$\frac{\vec{J}}{\stackrel{g}{\vec{g}}}$ & 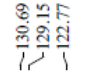 & $\begin{array}{l}\hat{c} \\
\hat{\infty} \\
1\end{array}$ & 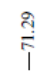 & 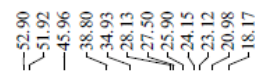 \\
\hline
\end{tabular}

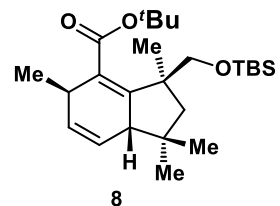

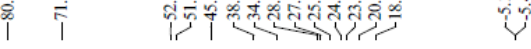

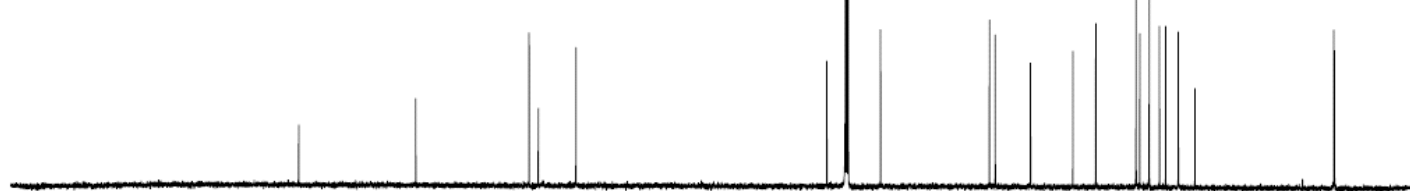

$\begin{array}{llllllllllllllllllllllll}210 & 200 & 190 & 180 & 170 & 160 & 150 & 140 & 130 & 120 & 110 & 100 & 90 & 80 & 70 & 60 & 50 & 40 & 30 & 20 & 10 & 0 & -10\end{array}$ 

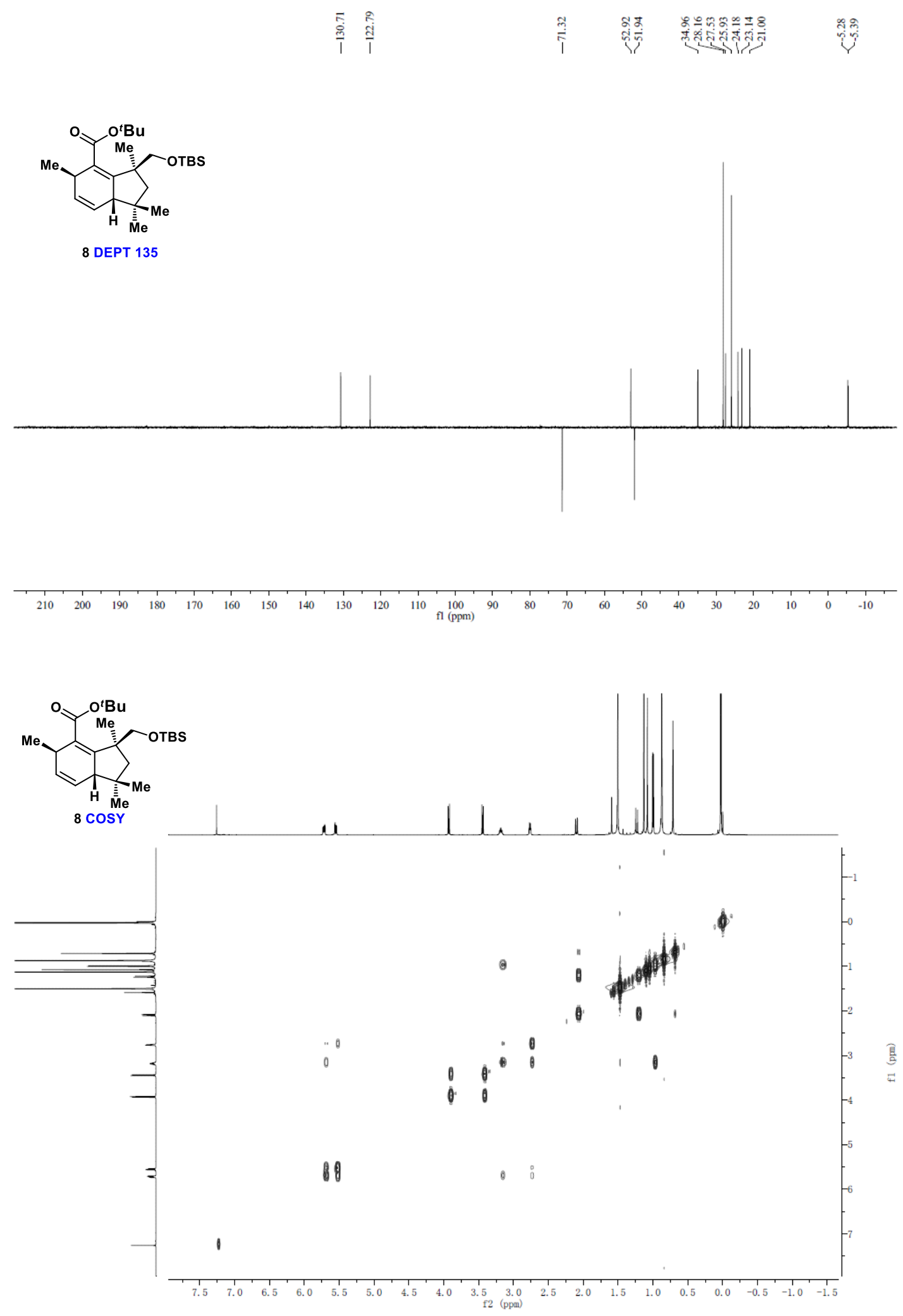

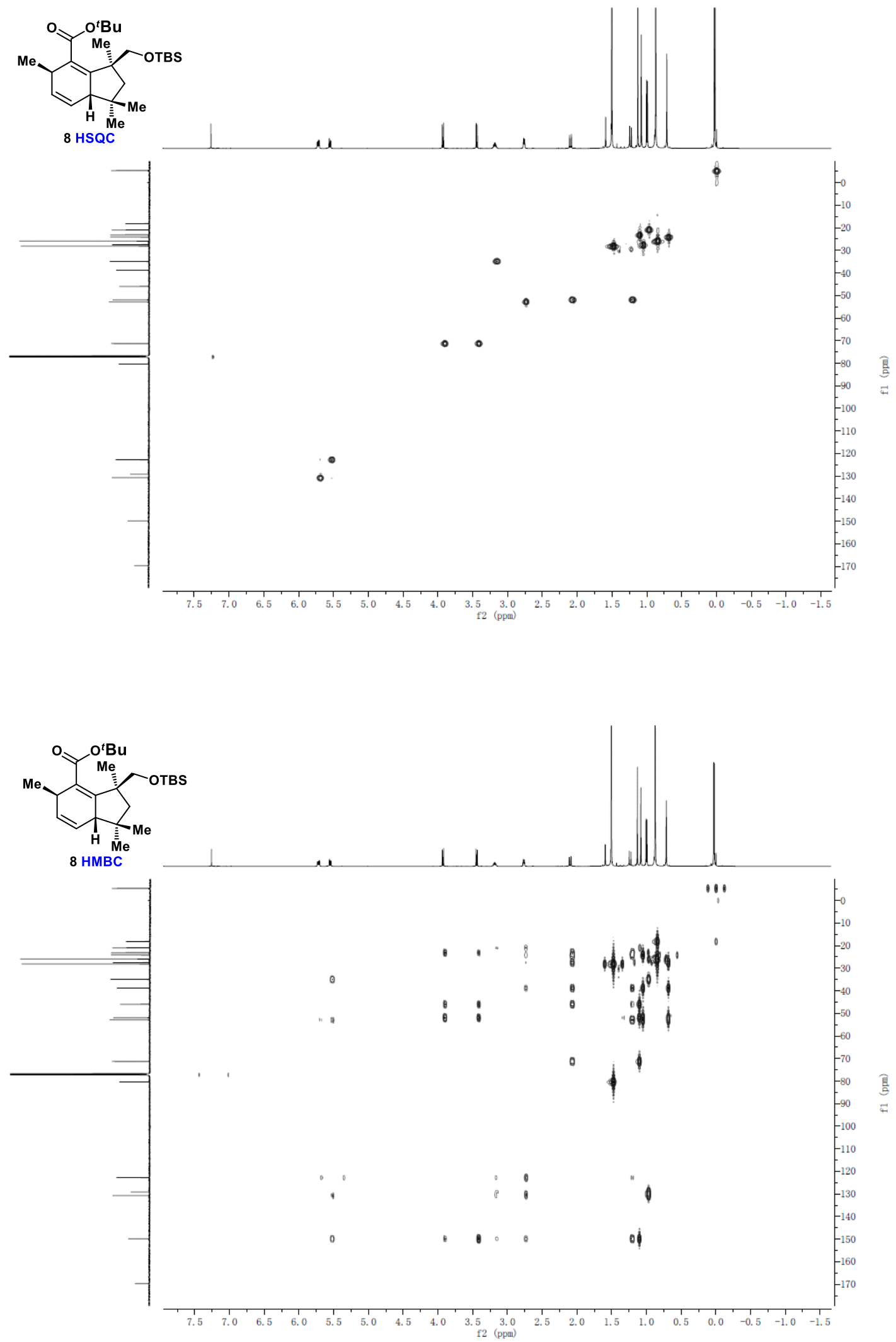


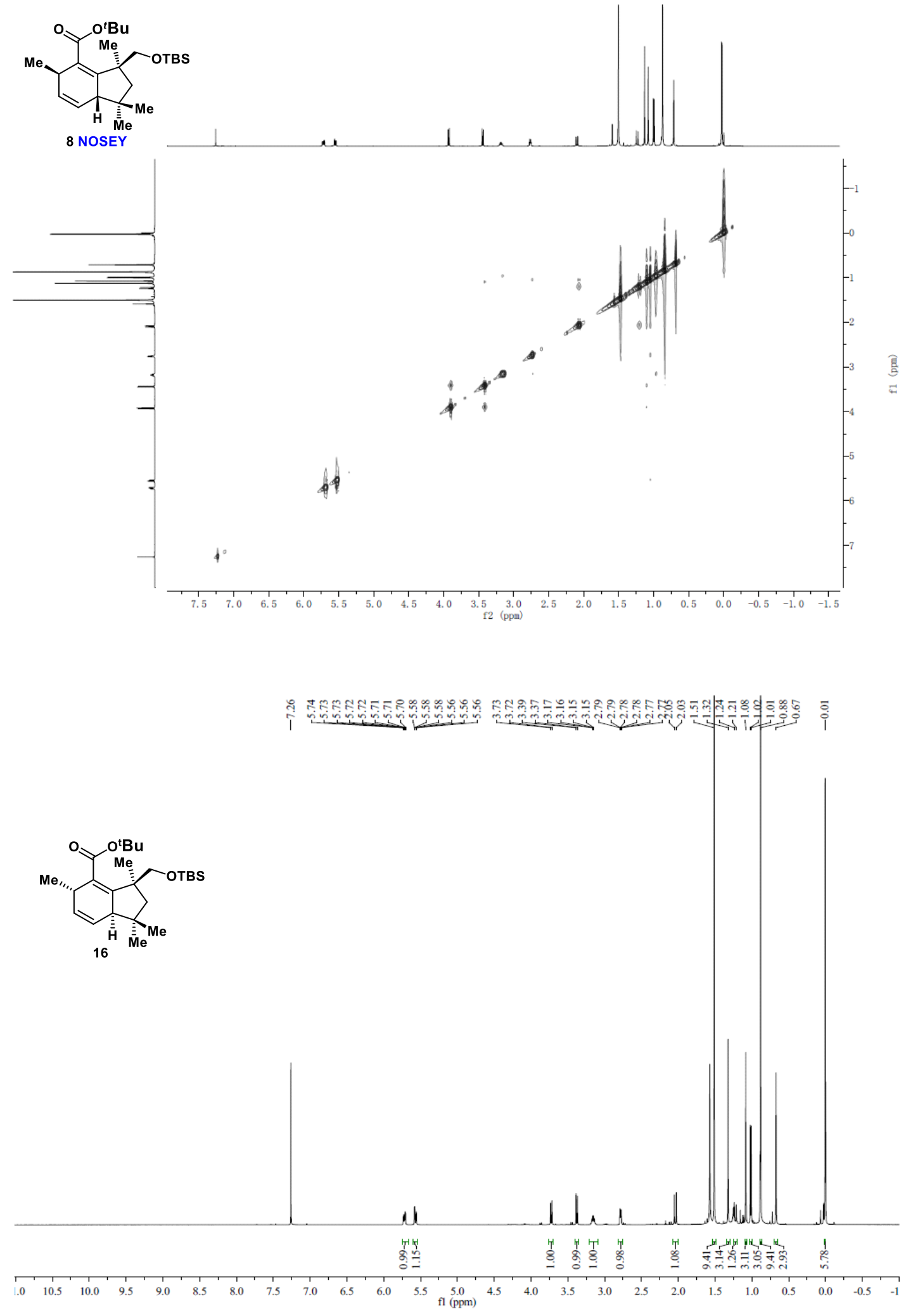



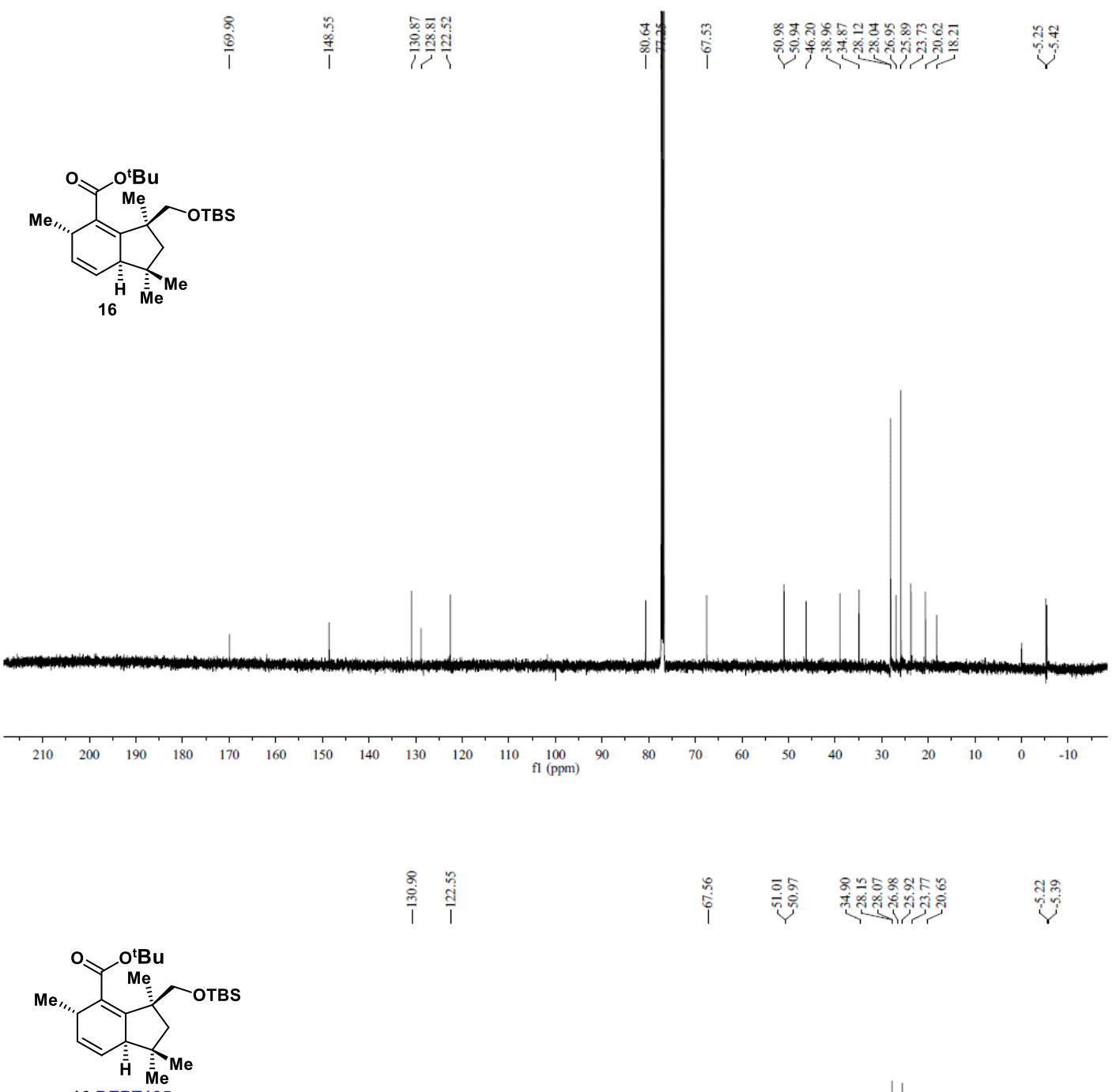

16 DEPT135

$\begin{array}{llllllllllllllllllllllllll}1 & 210 & 200 & 190 & 180 & 170 & 160 & 150 & 140 & 130 & 120 & 110 & \begin{array}{c}100 \\ \mathrm{fl}(\mathrm{ppm})\end{array} & 90 & 80 & 70 & 60 & 50 & 40 & 30 & 20 & 10 & 0 & -10\end{array}$ 

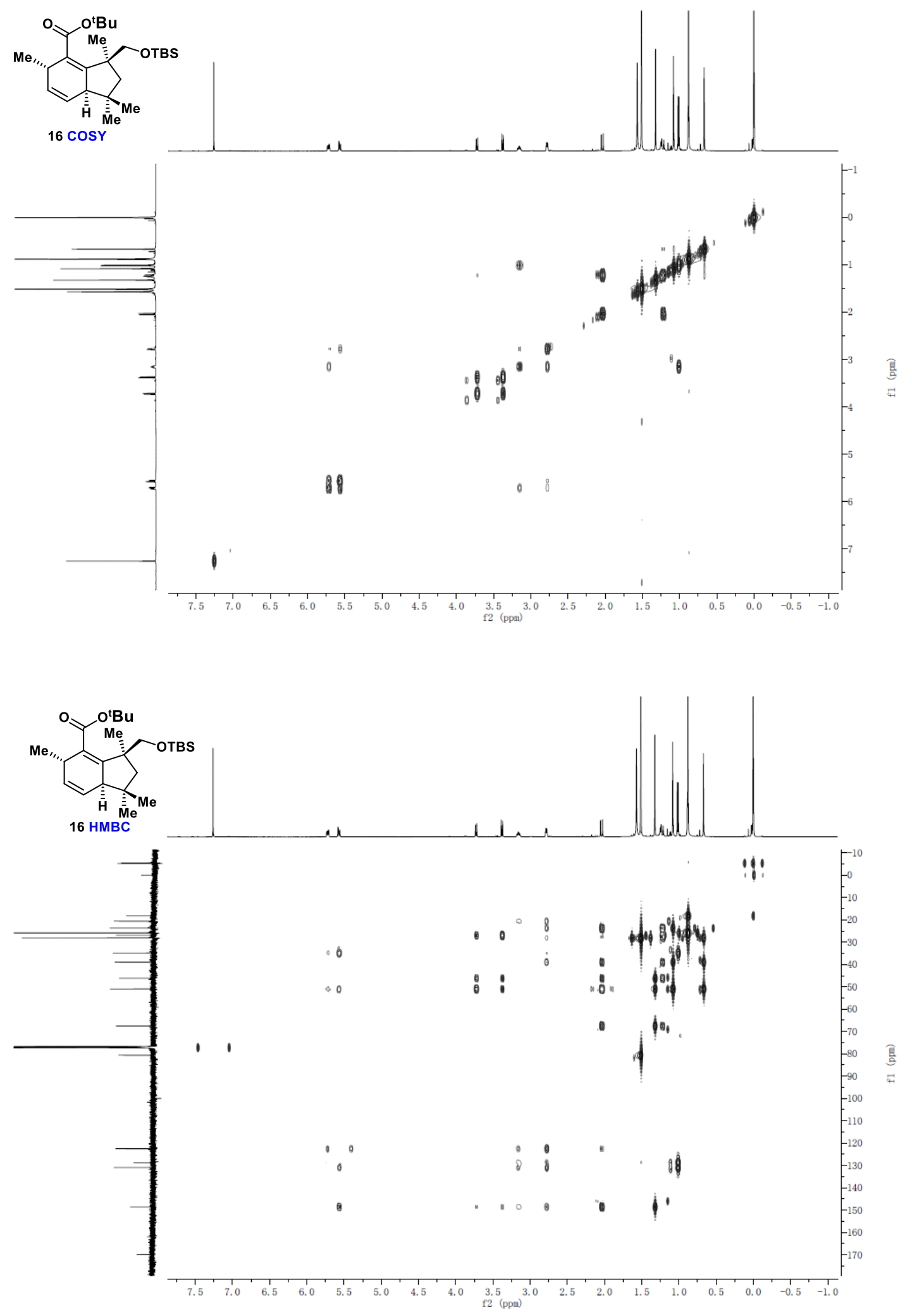


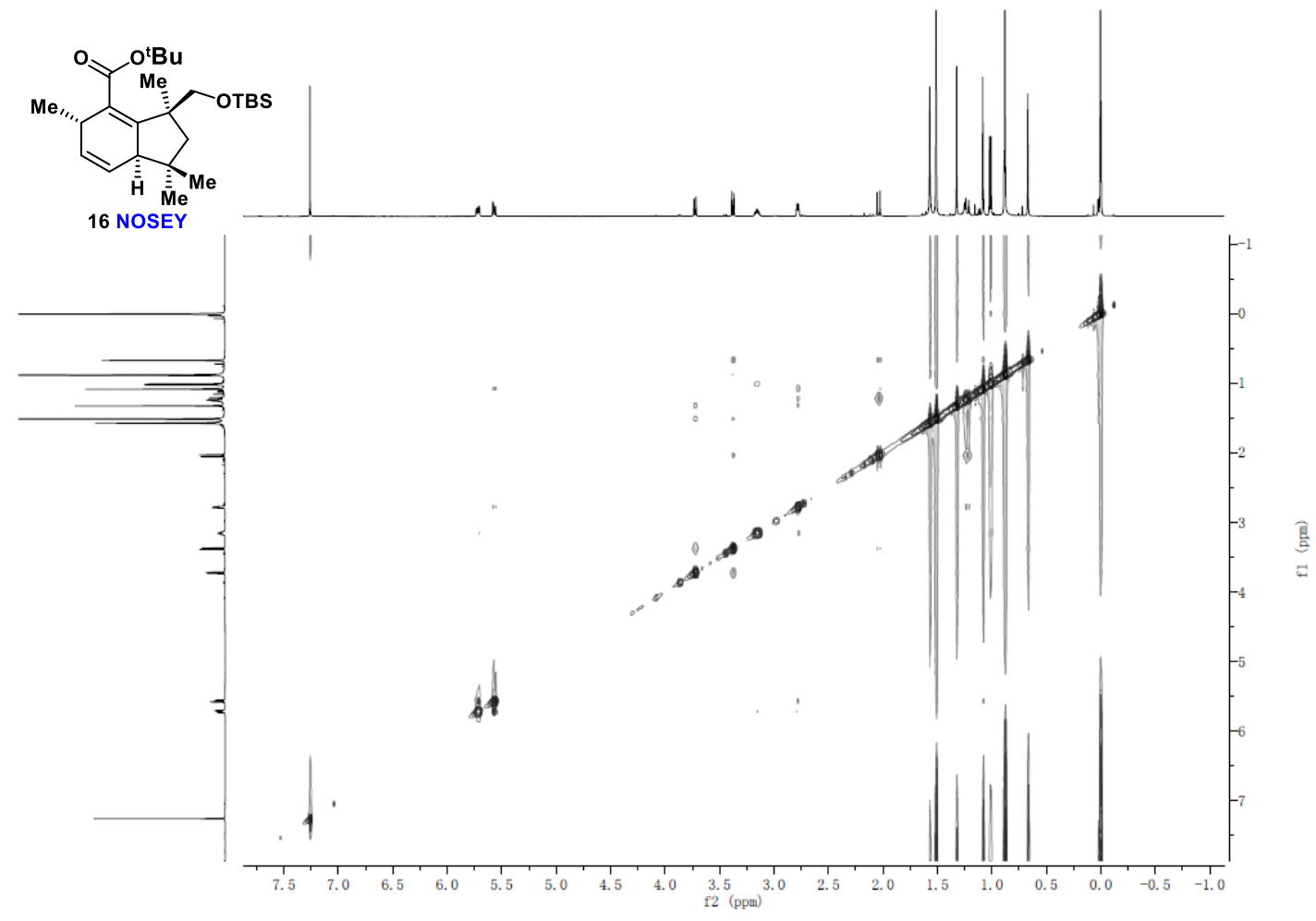

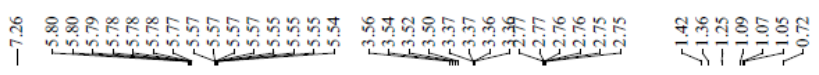
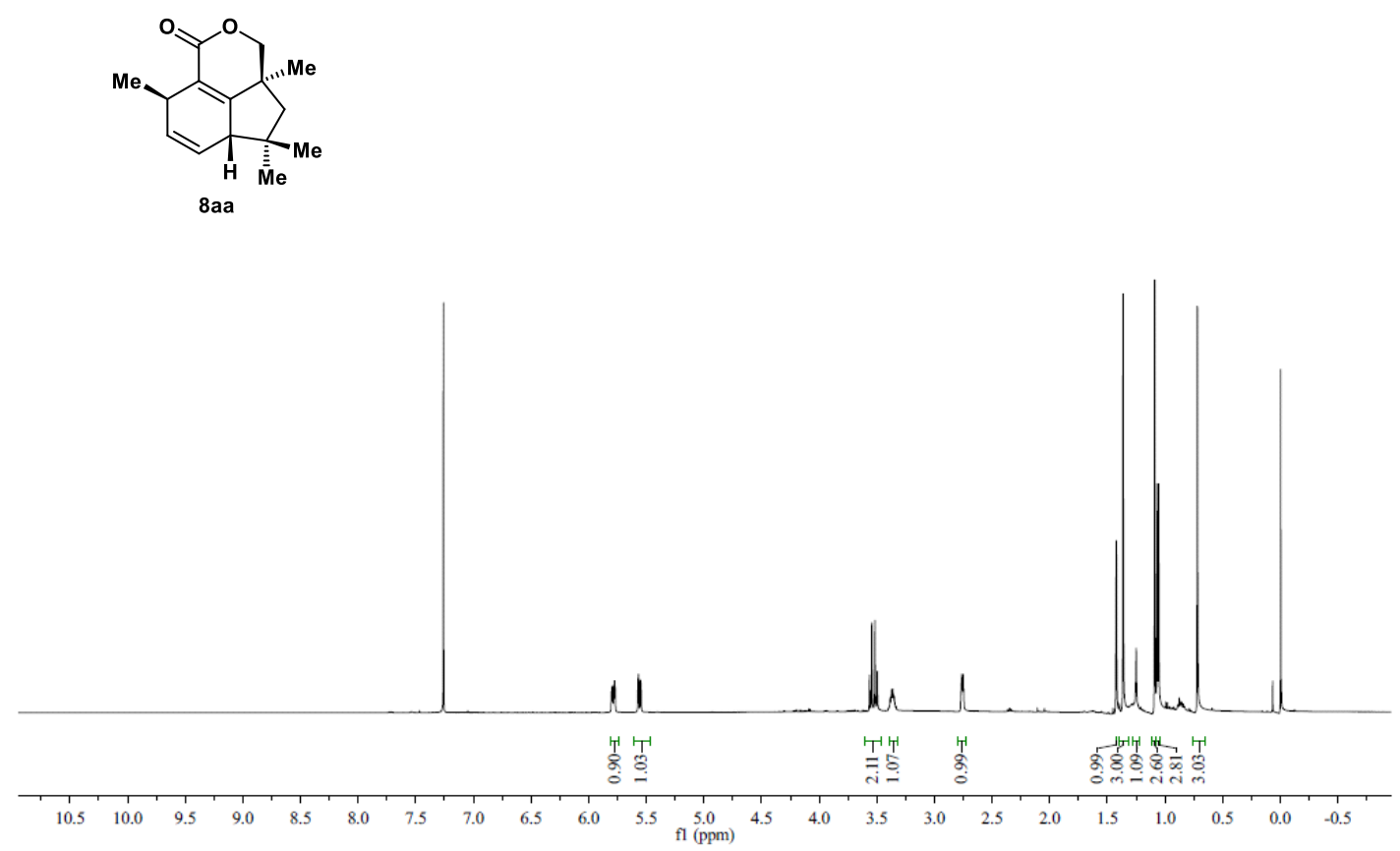

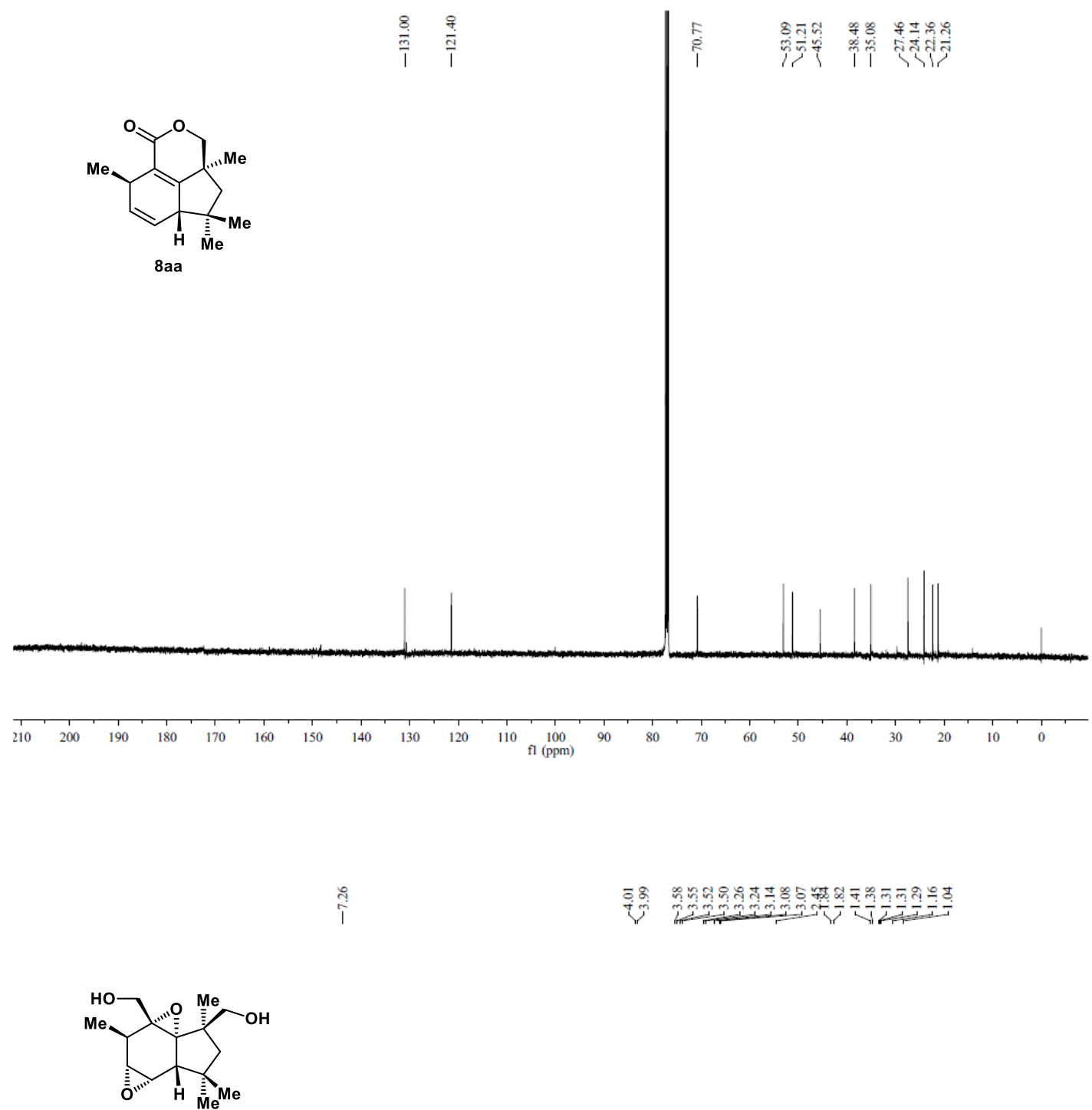

$8 a$

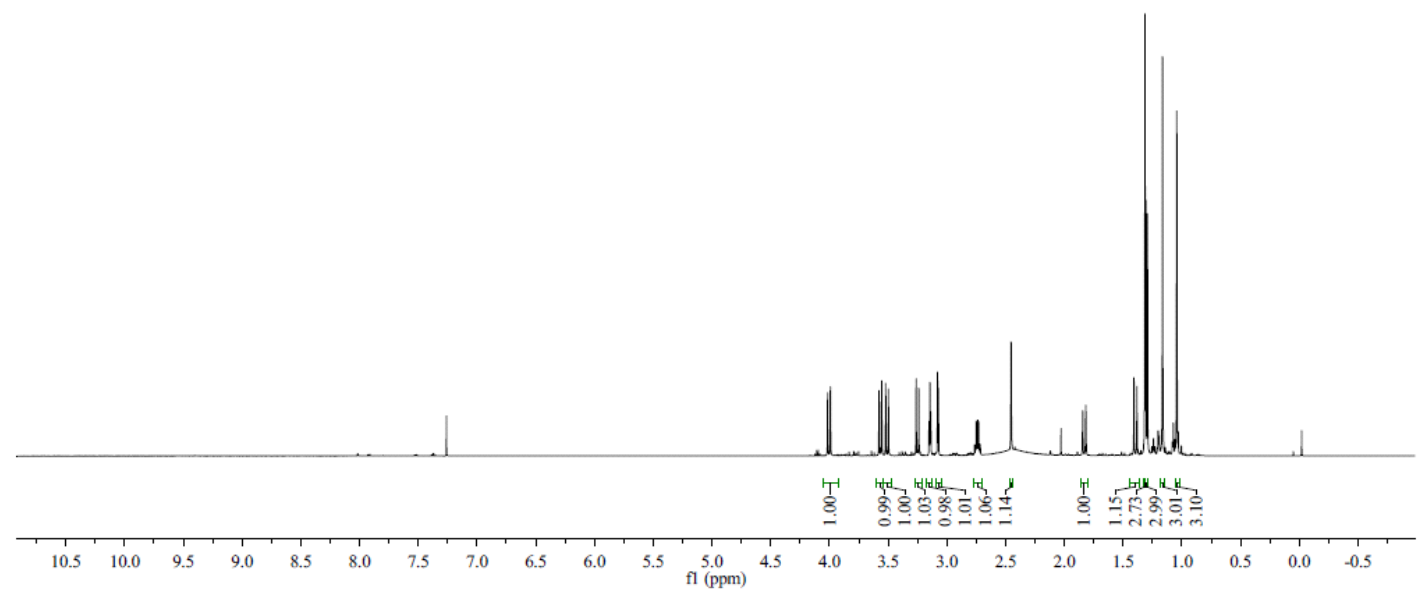




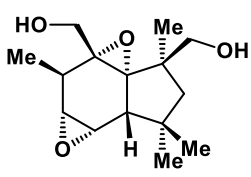

$8 a$
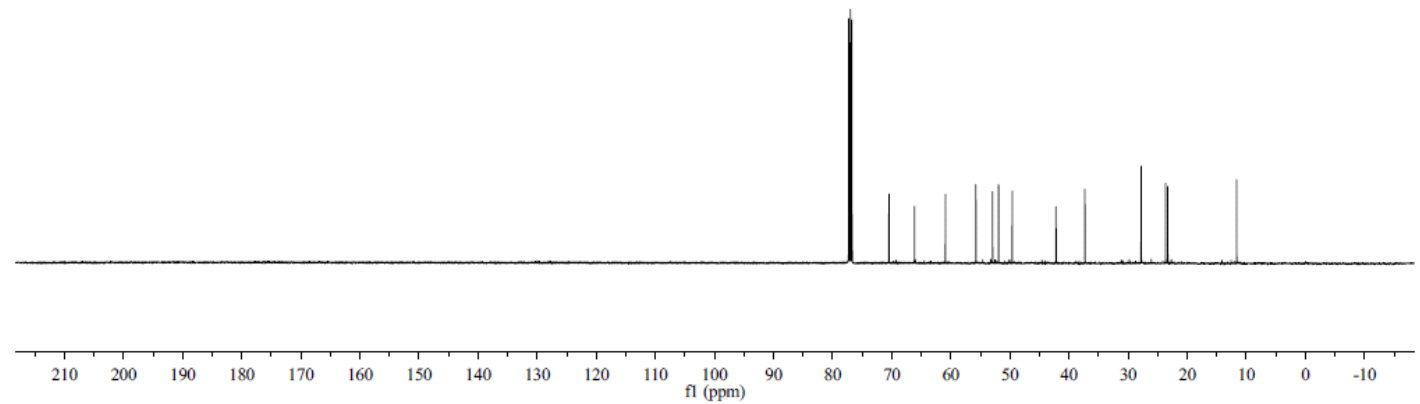

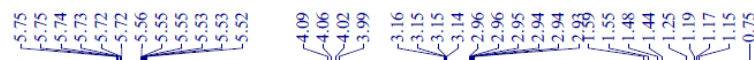

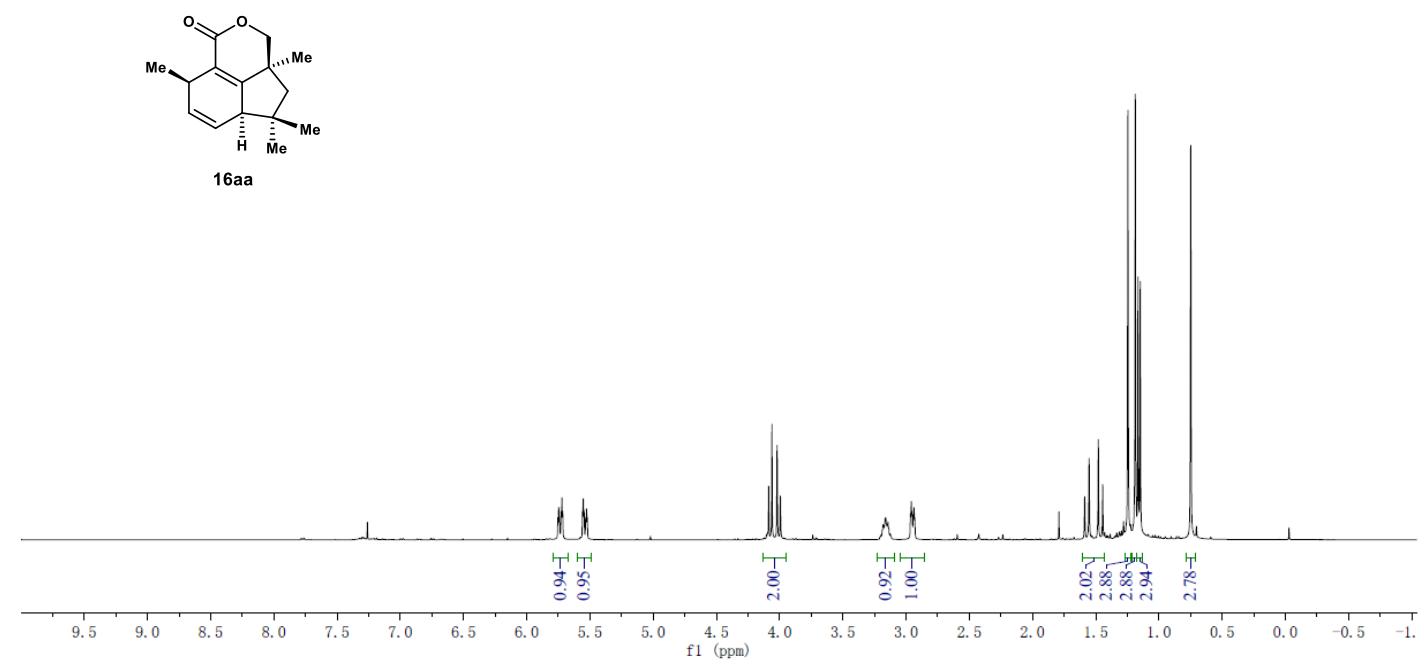



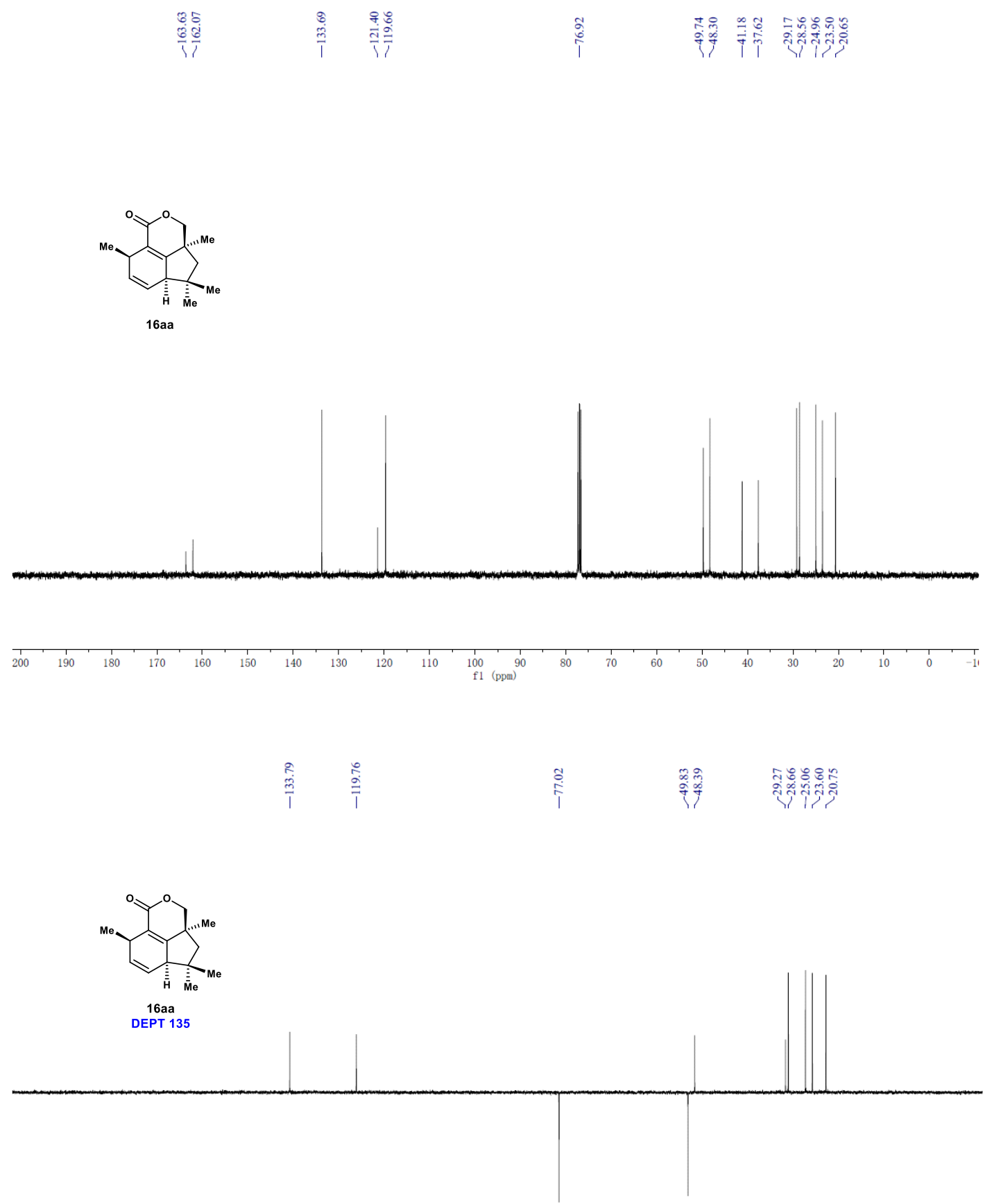

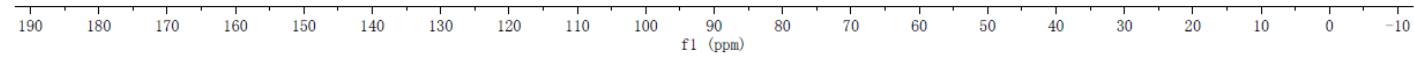



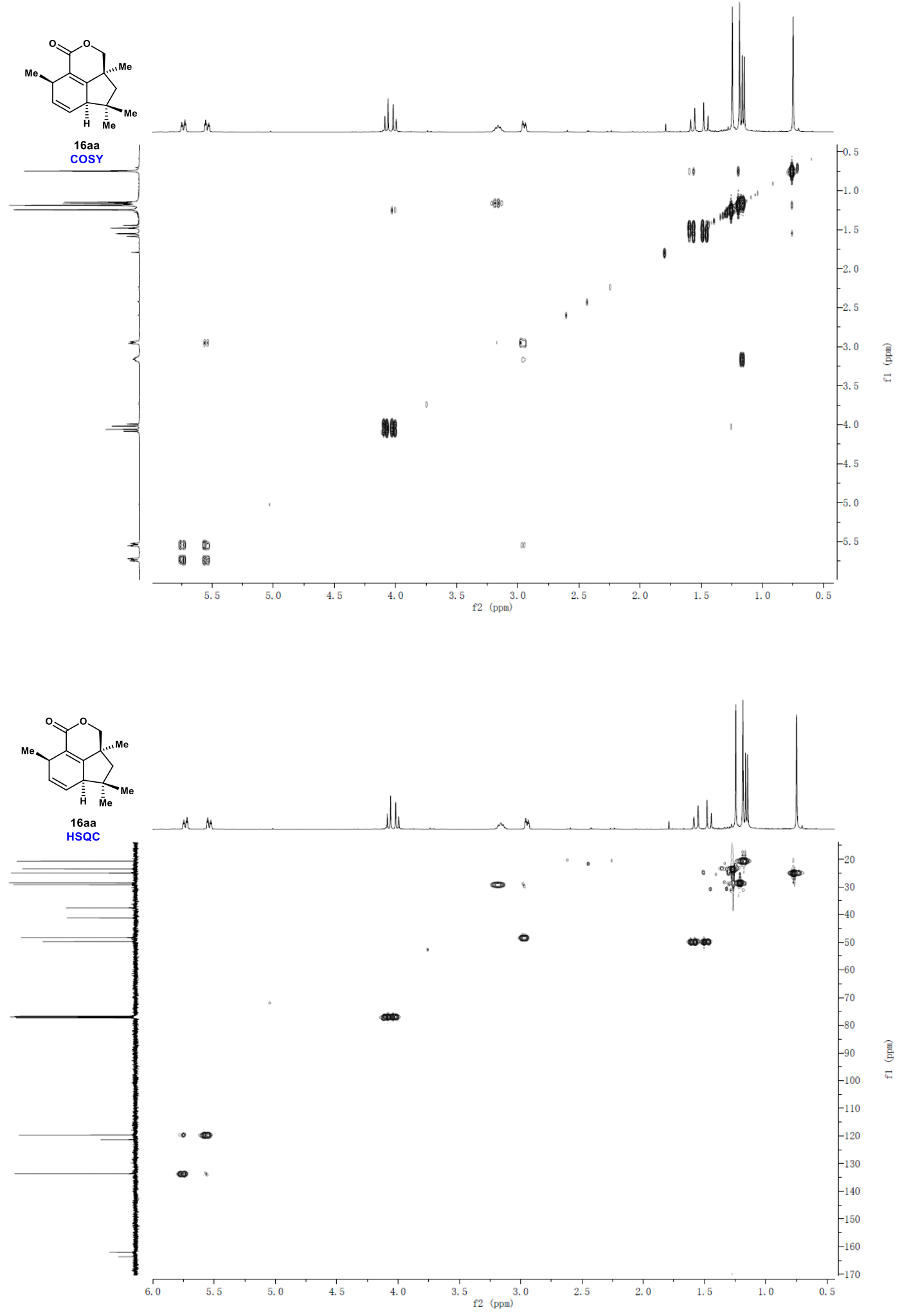

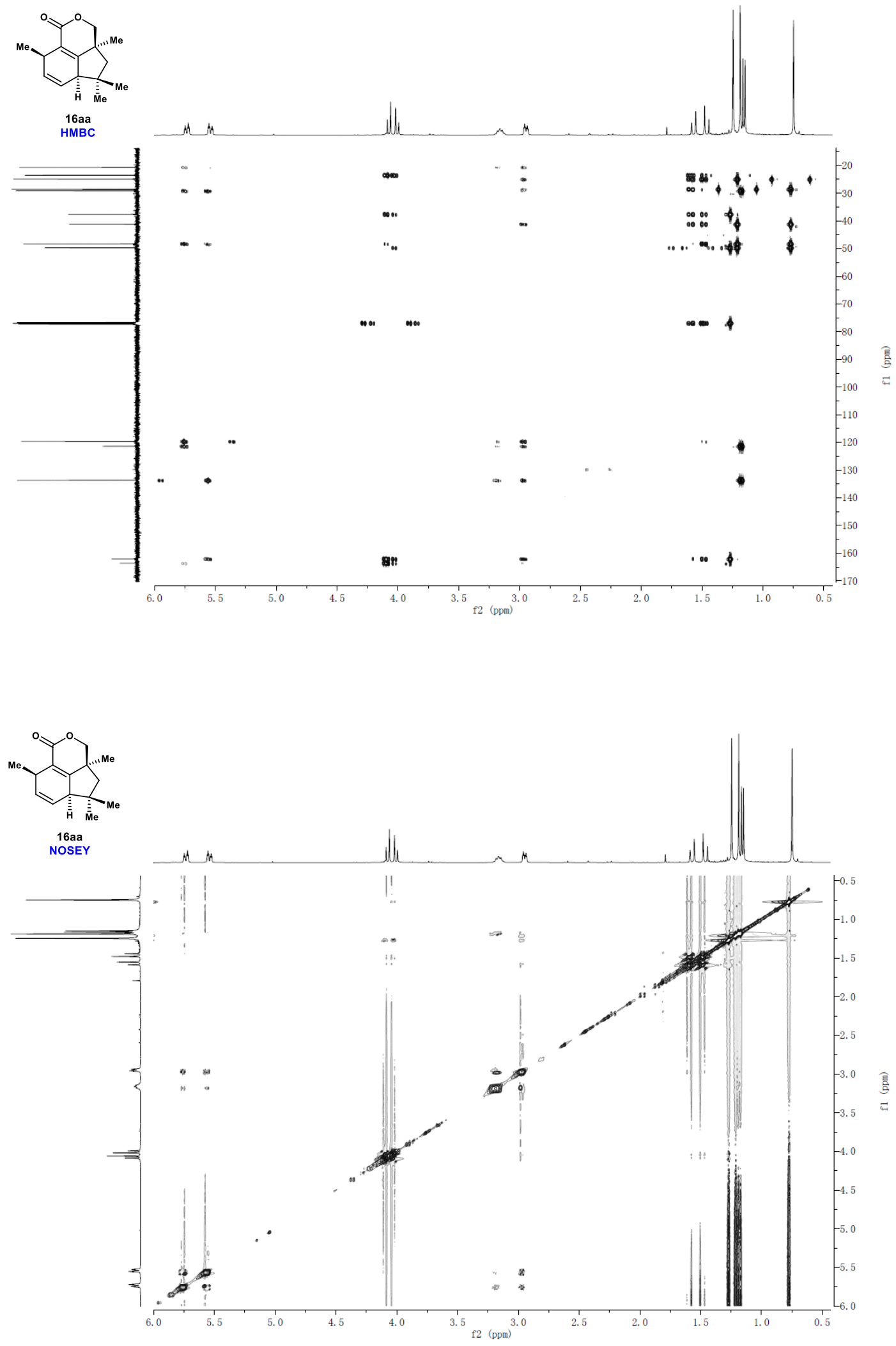


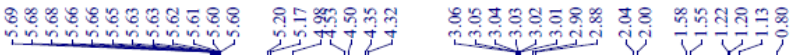

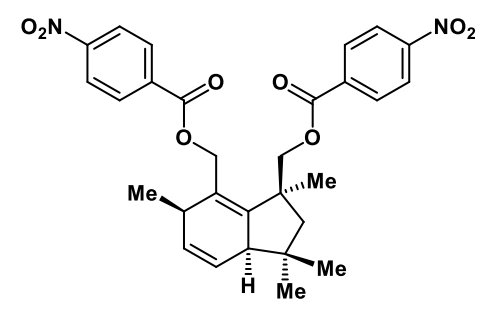

$16 \mathrm{~b}$
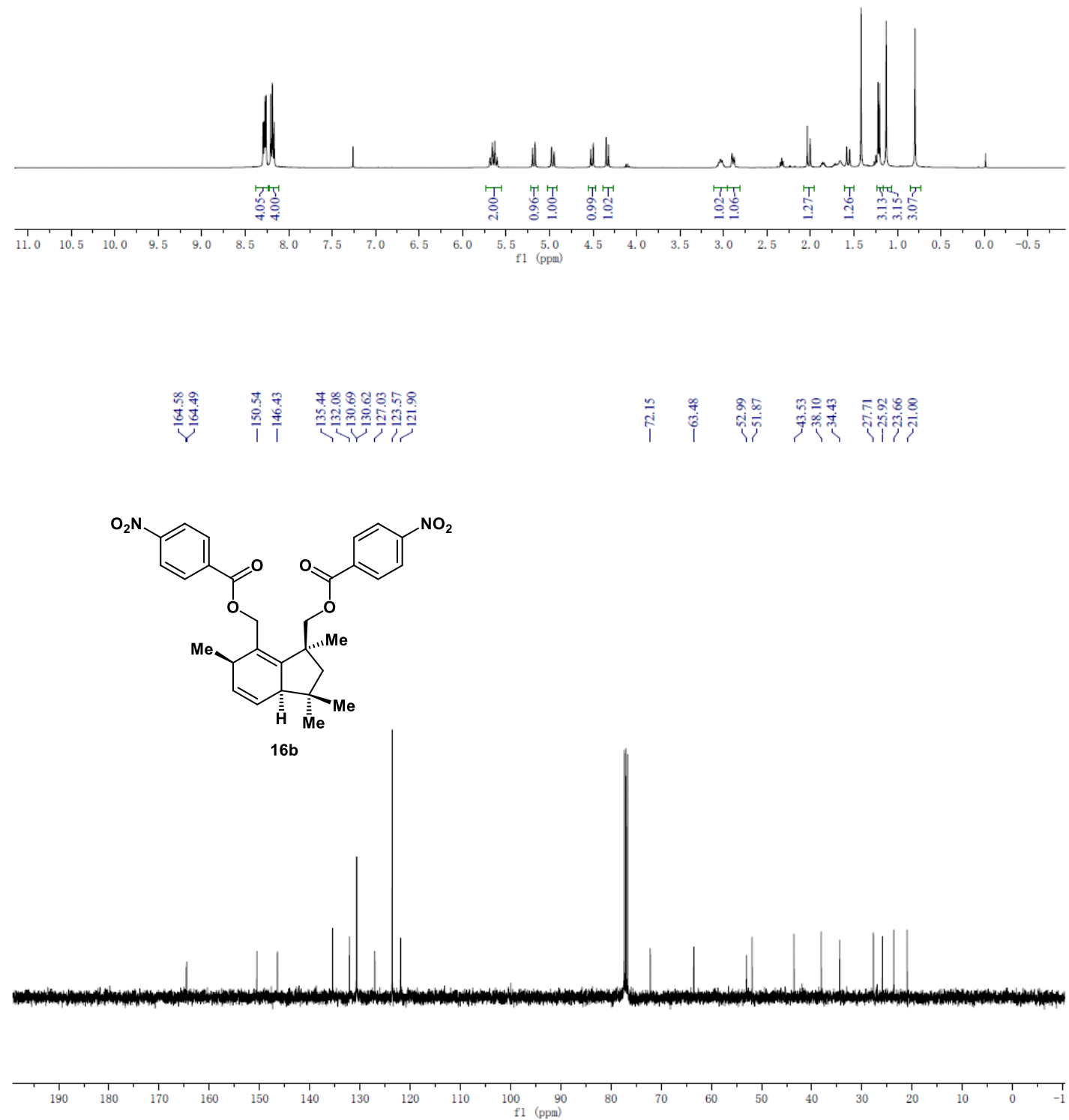


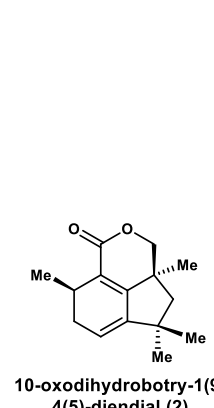

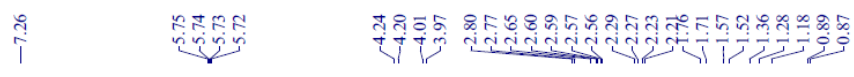

4(5)-diendial (2)

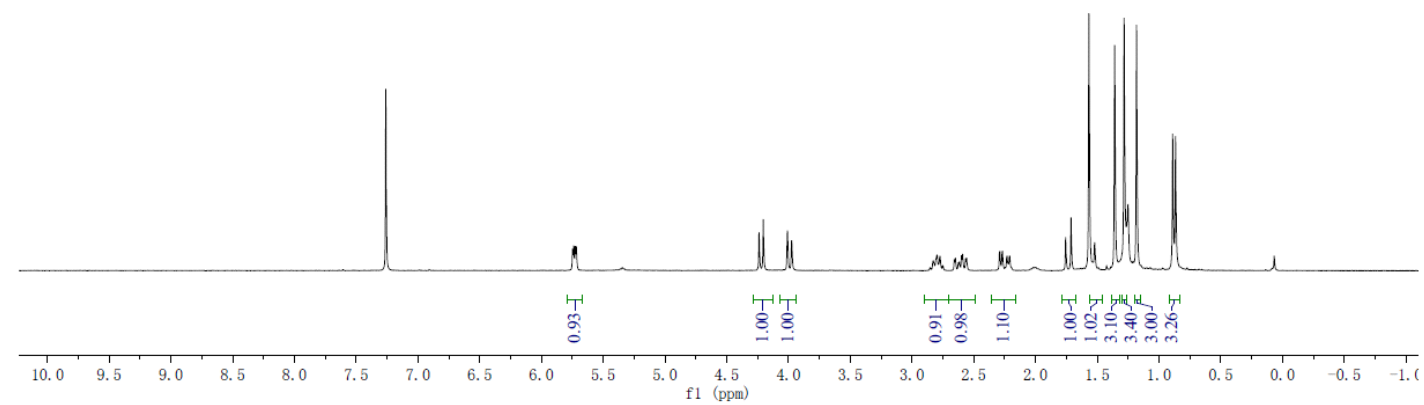

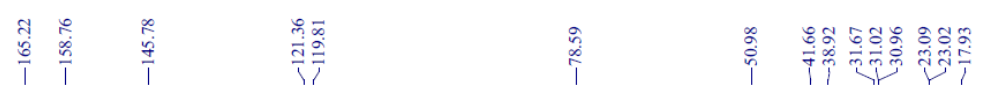

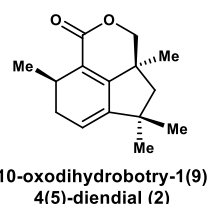

4(5)-diendial (2)
-oxodihydrobotry-1(9)

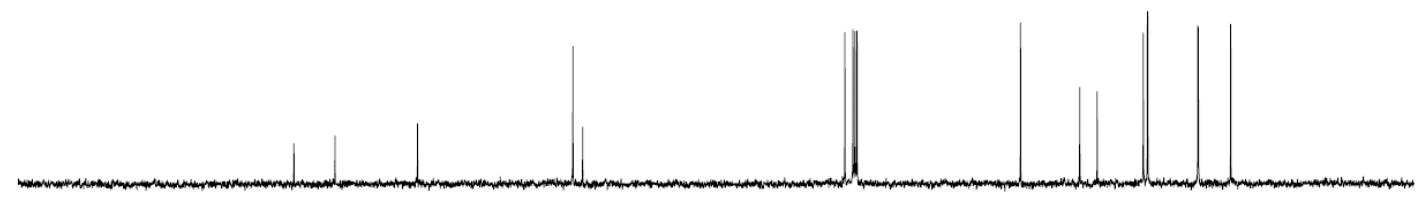

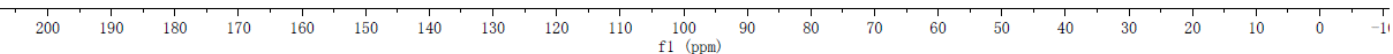




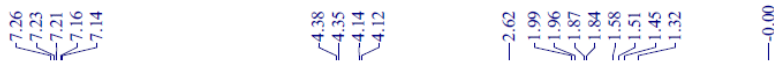

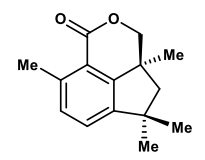

10-oxodehydro-dihydro-

botrydial (5)

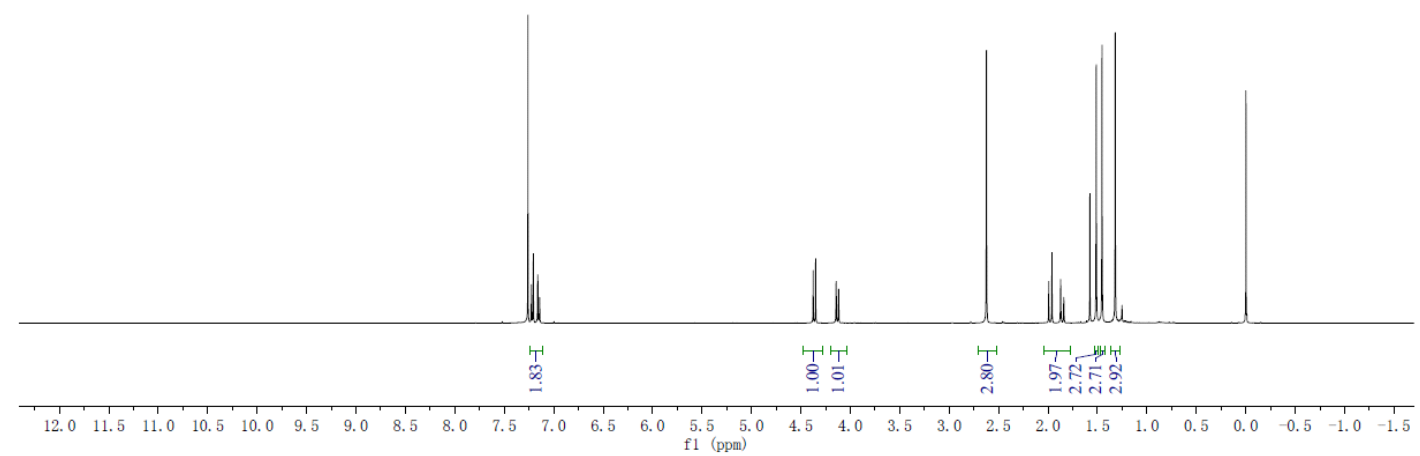

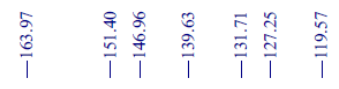

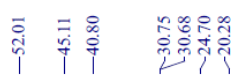

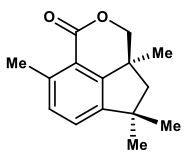

10-oxodehydro-dihydro-

botrydial (5)

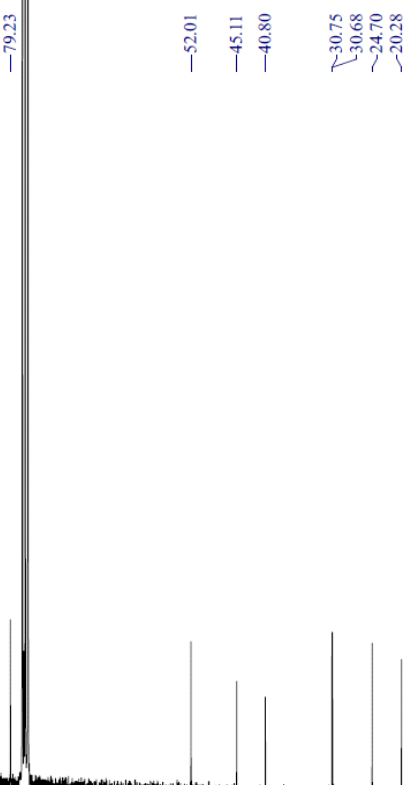

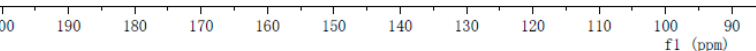


$\stackrel{\text { îd }}{\stackrel{1}{2}}$

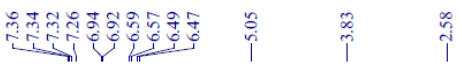

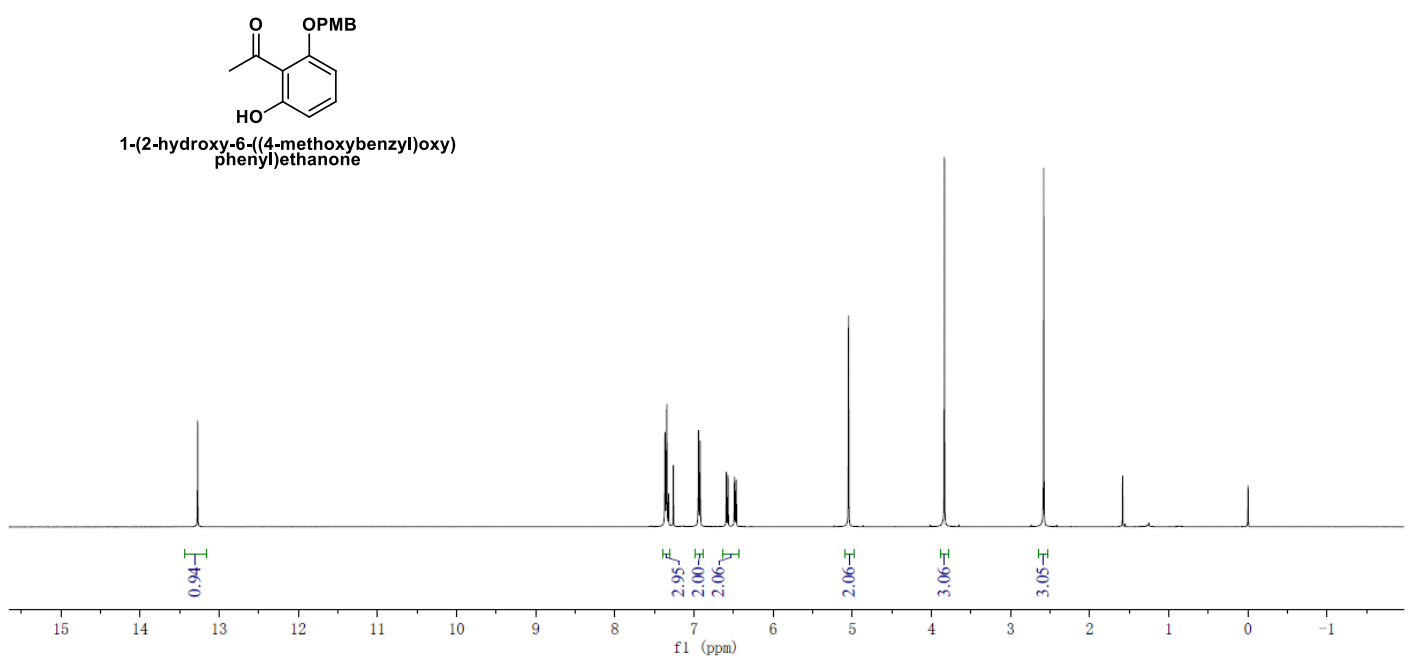

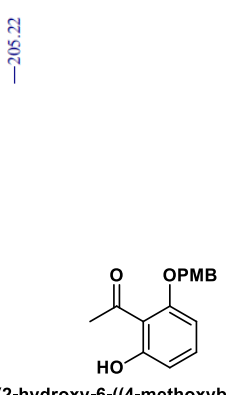

1-(2-hydroxy-6-((4-methoxybenzyl)oxy)
phenyl)ethanone
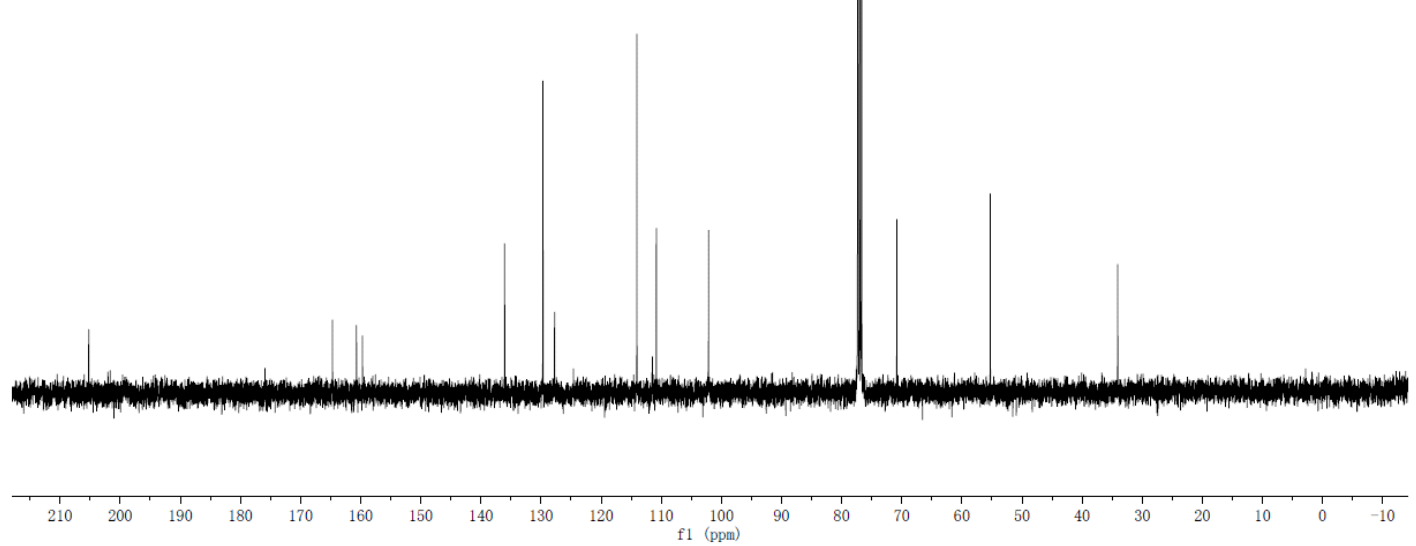

S57 


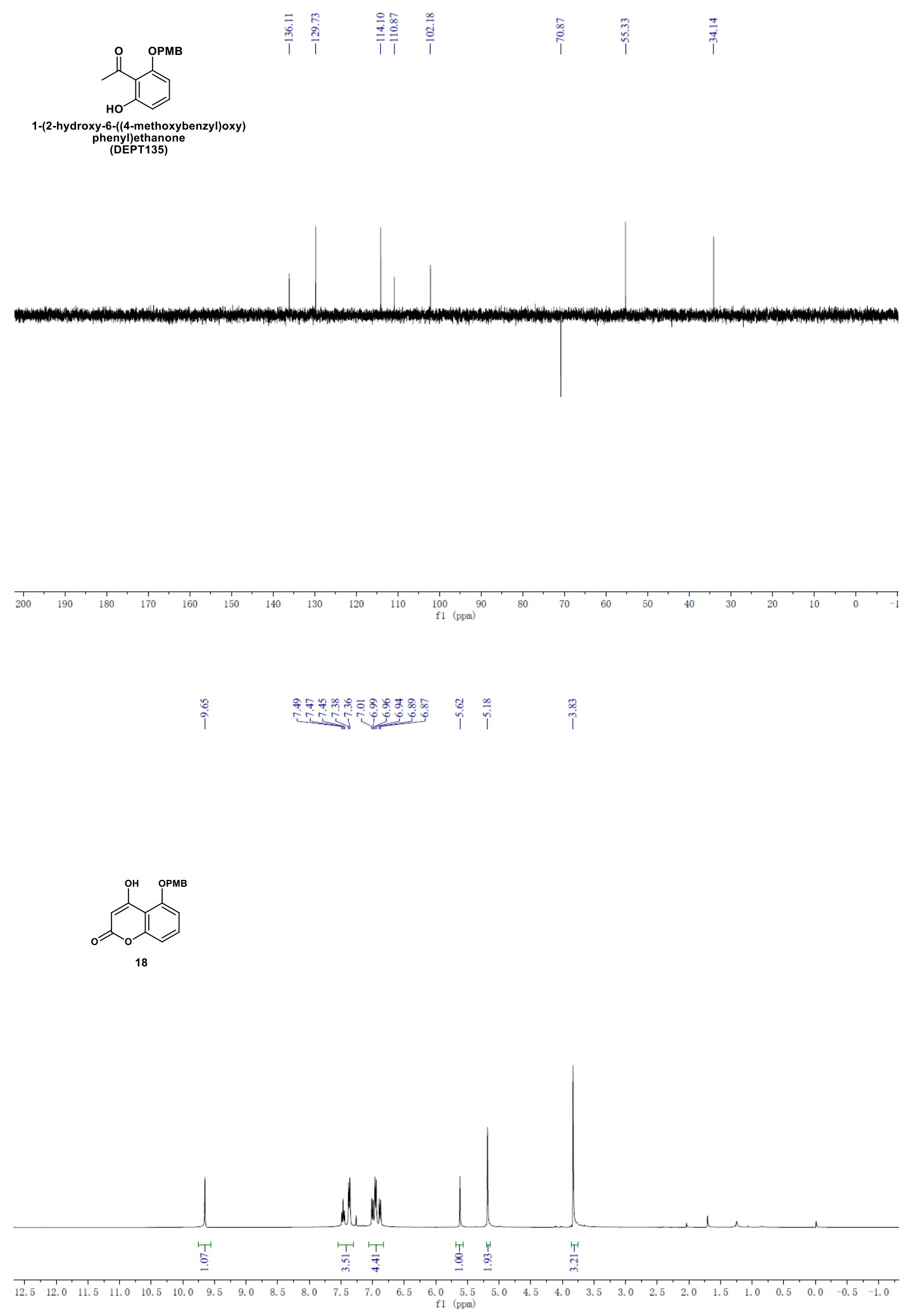




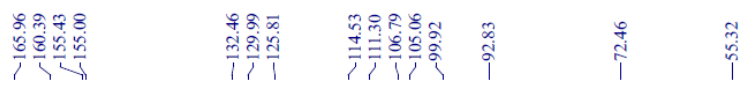<smiles>CCc1cccc2cc(C)cc(C)c12</smiles>

18
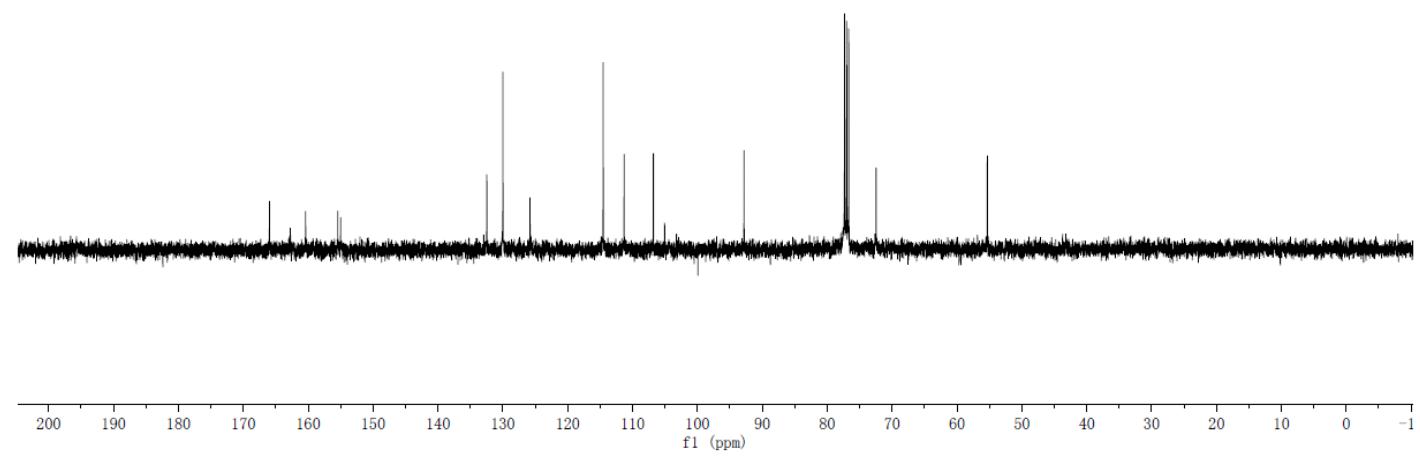

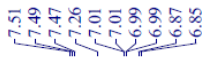

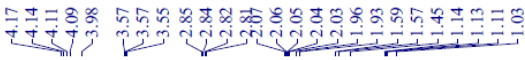

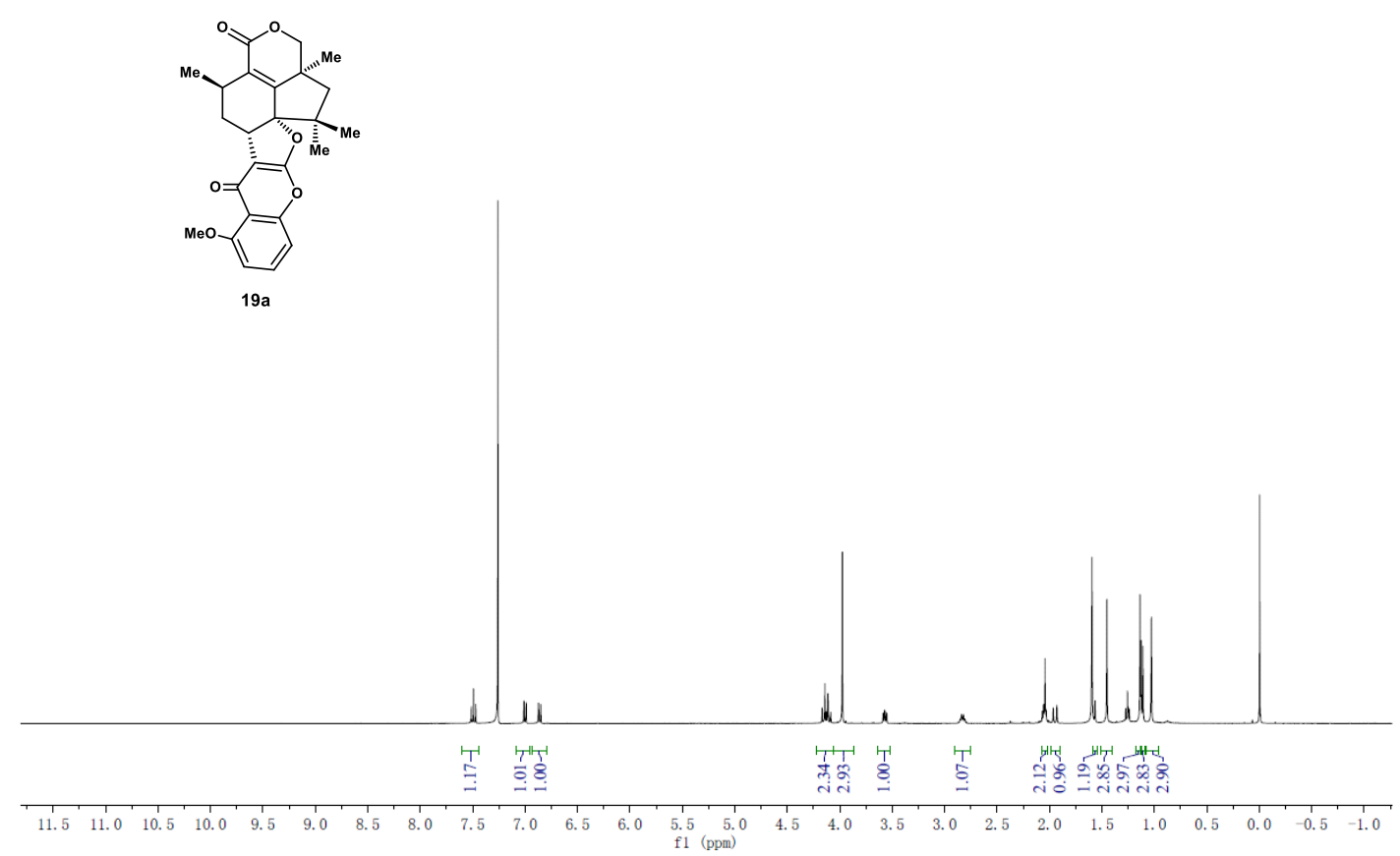



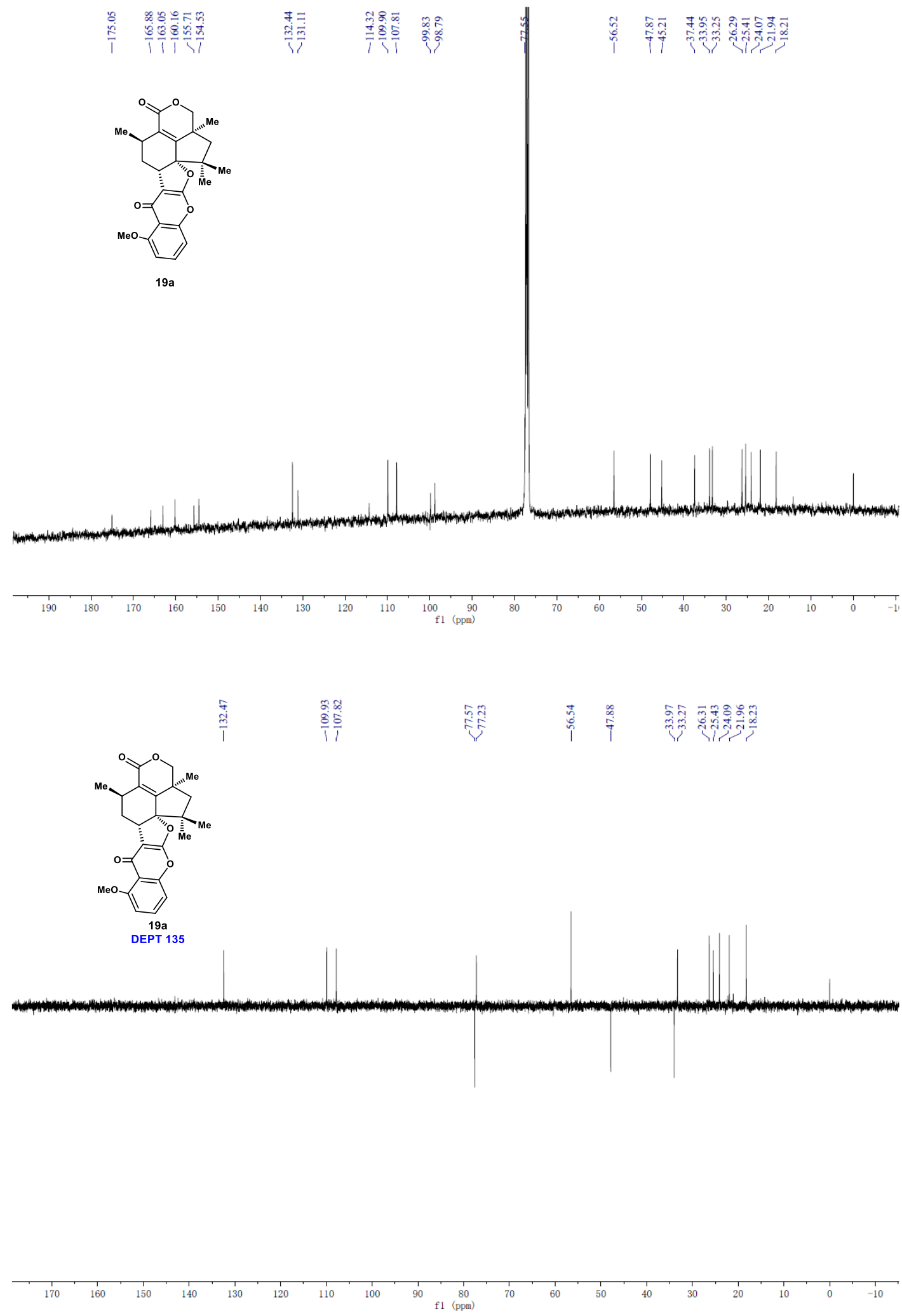

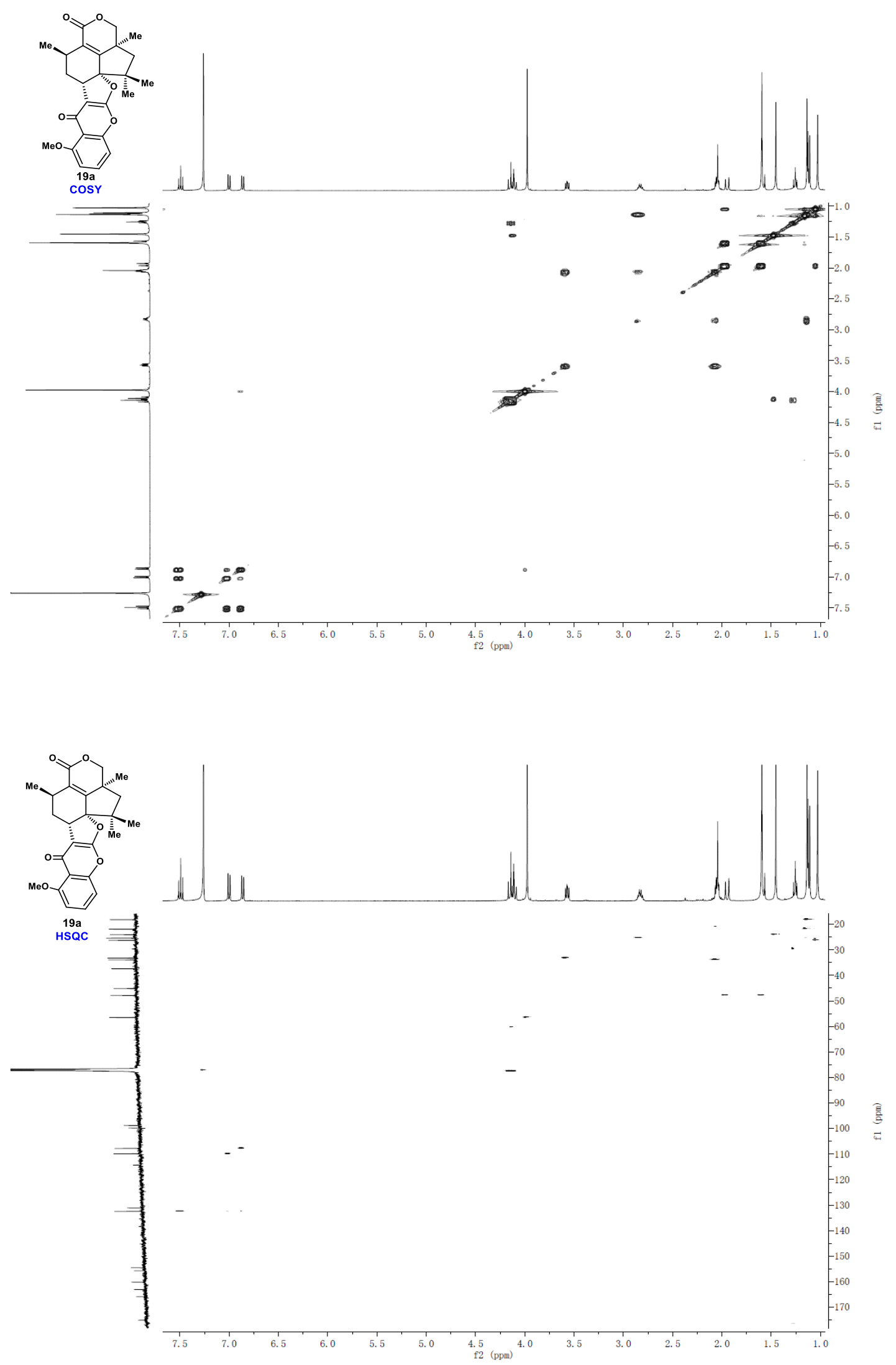

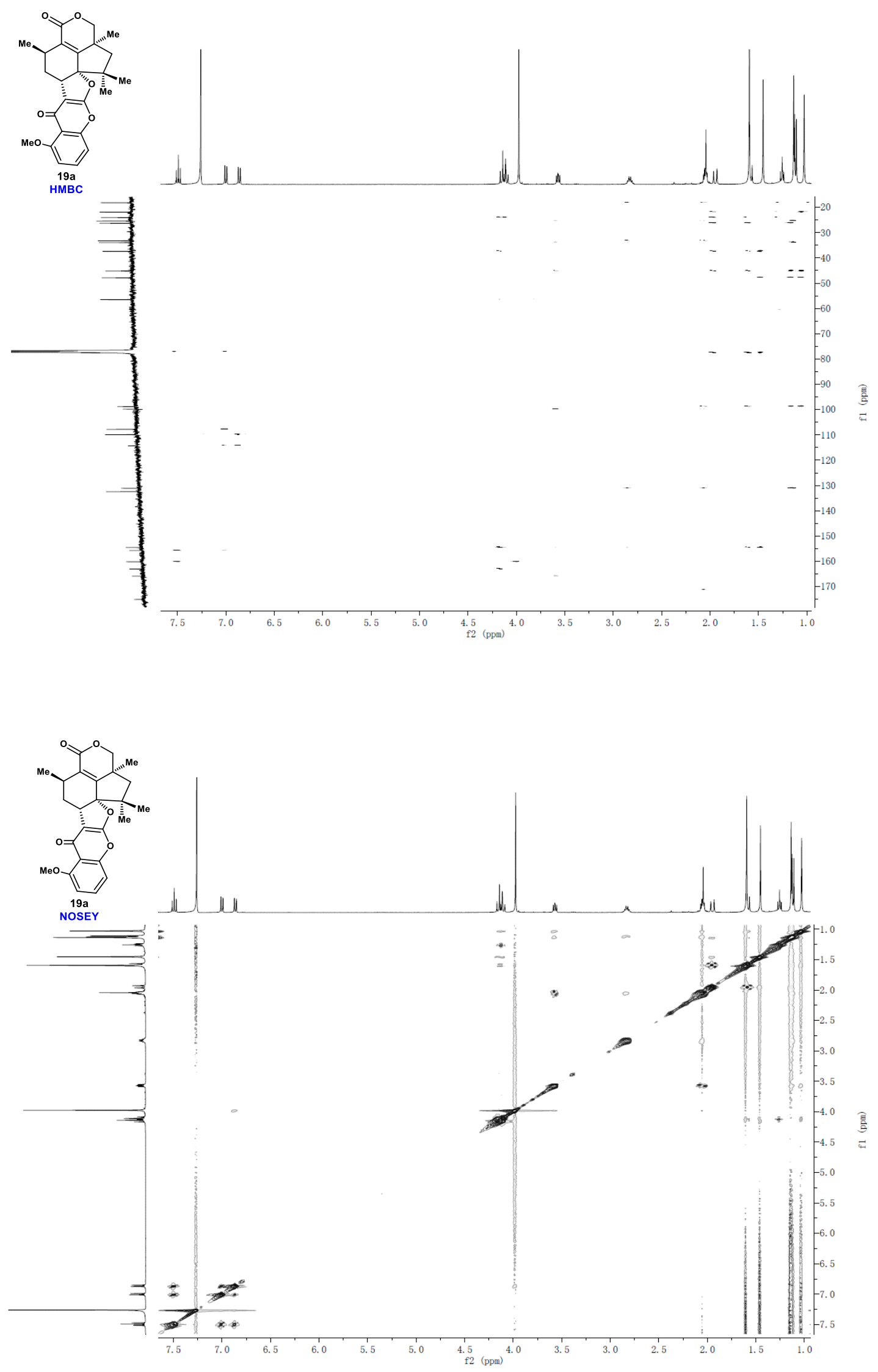

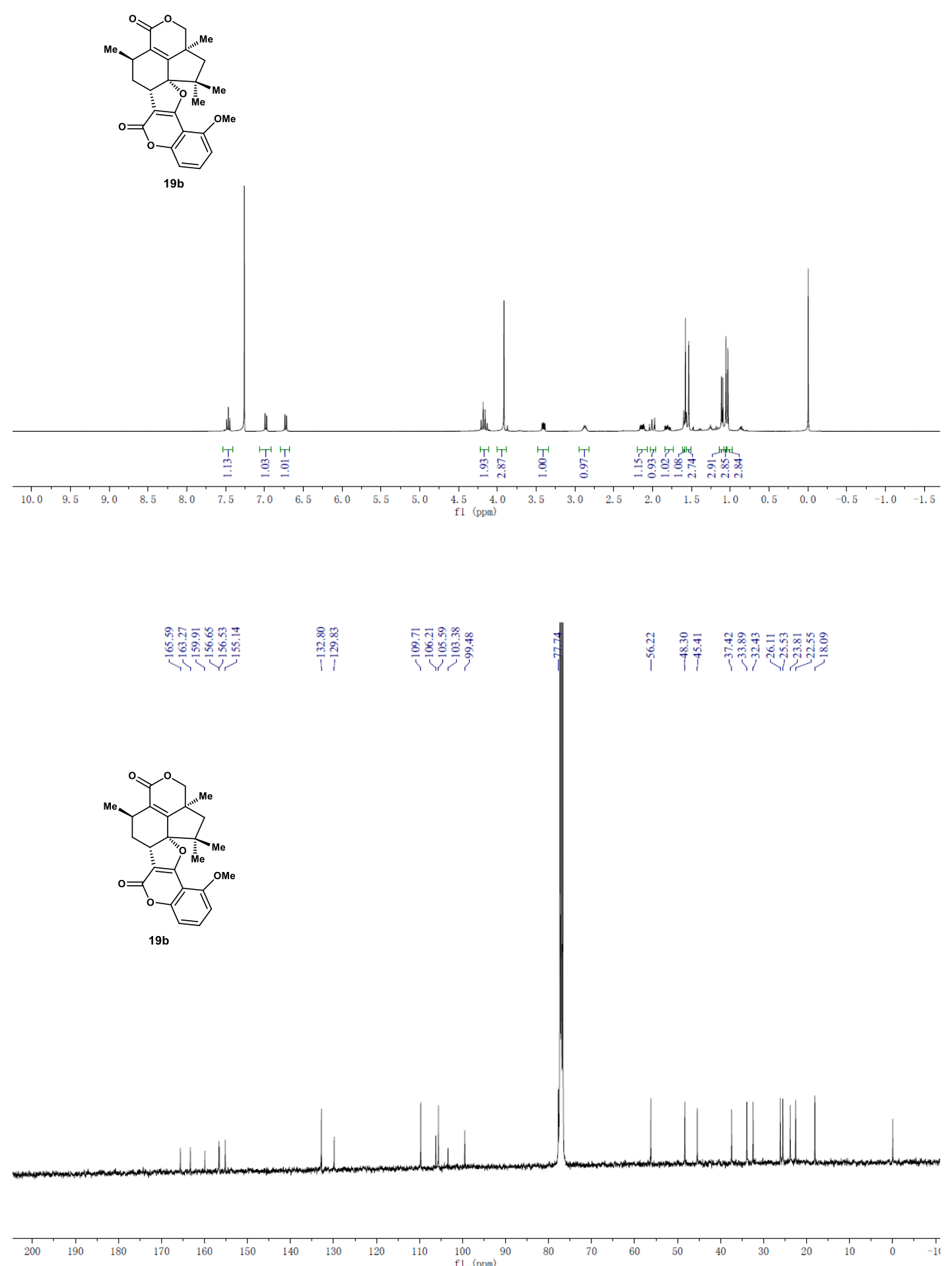

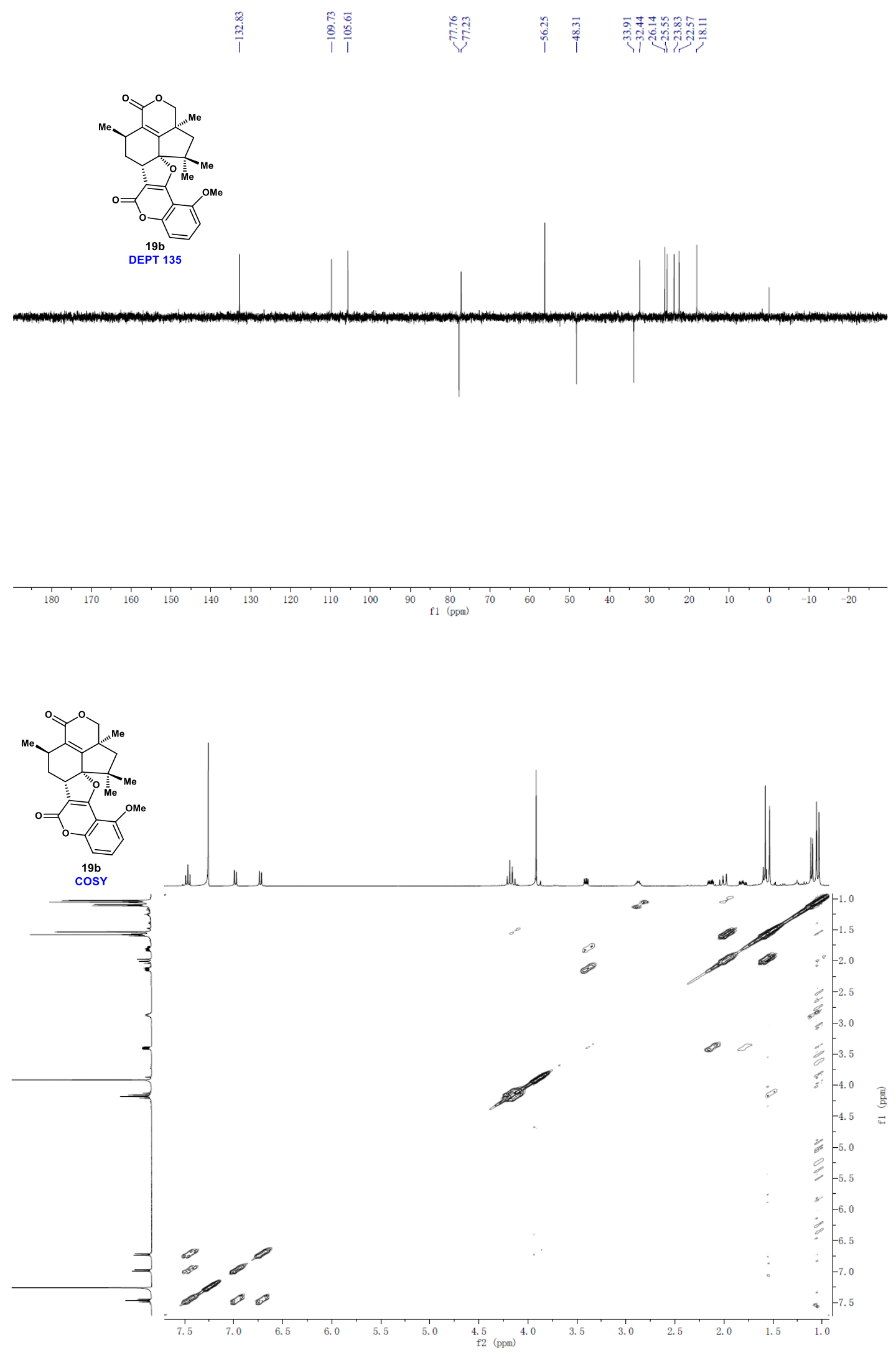

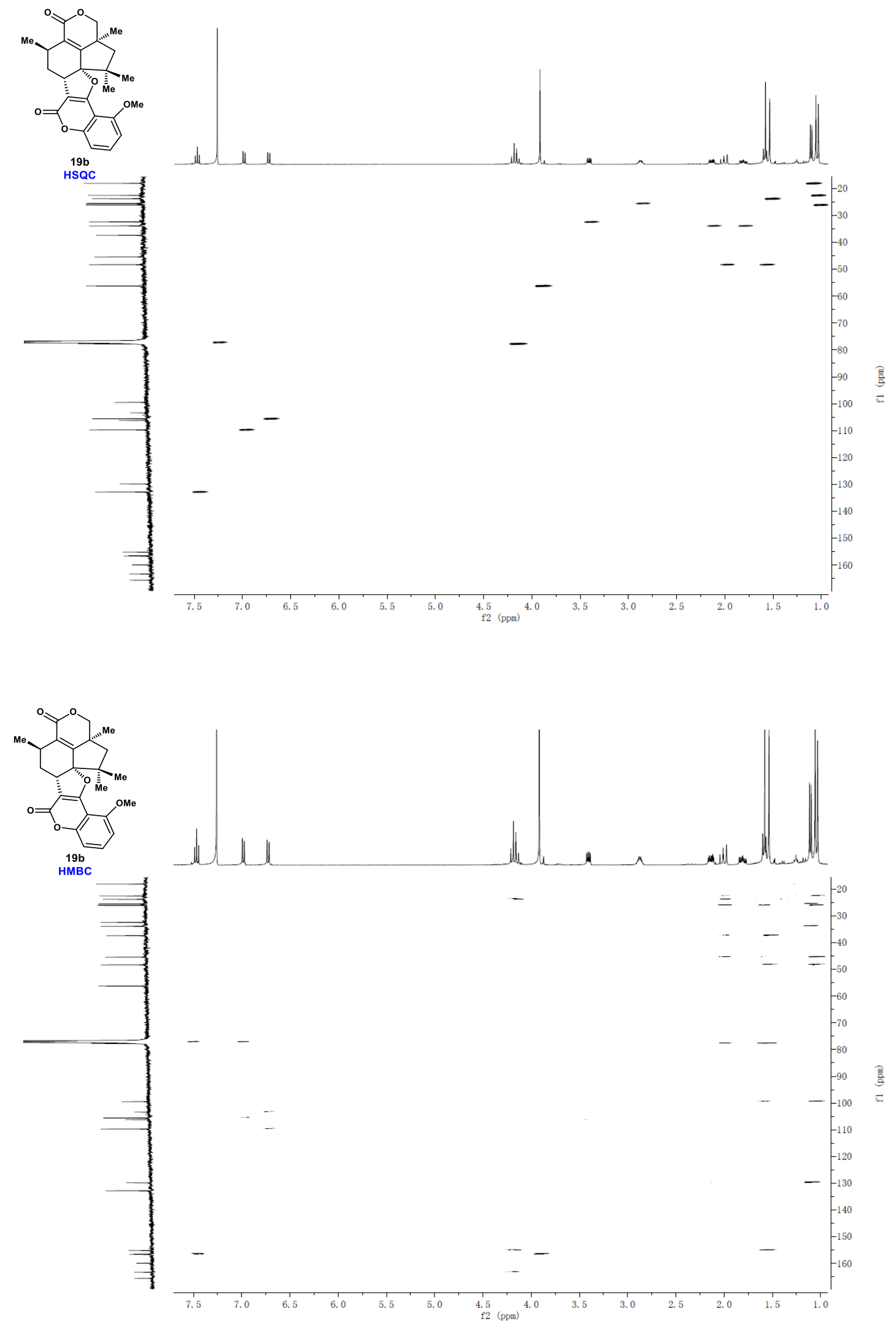

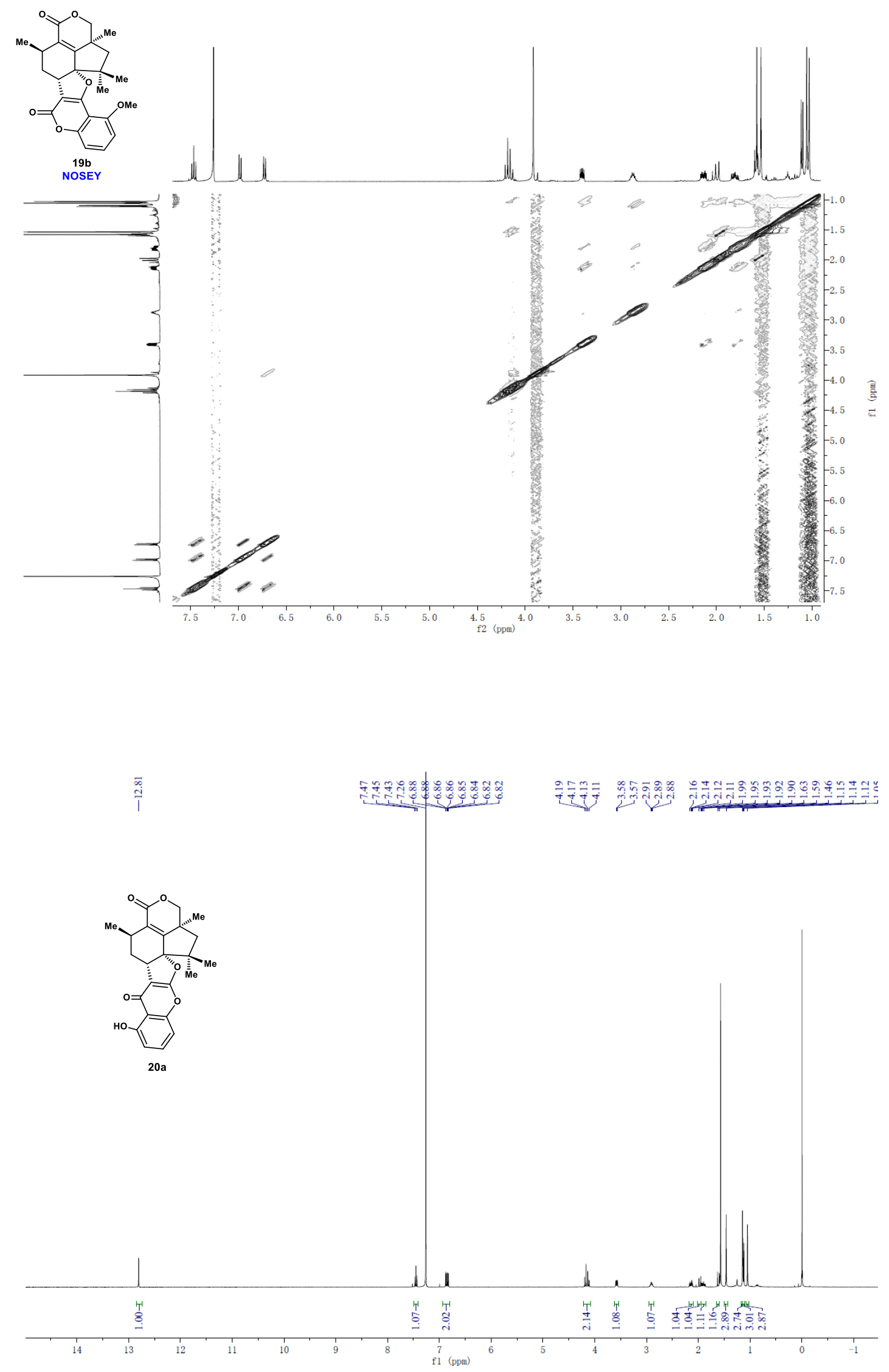


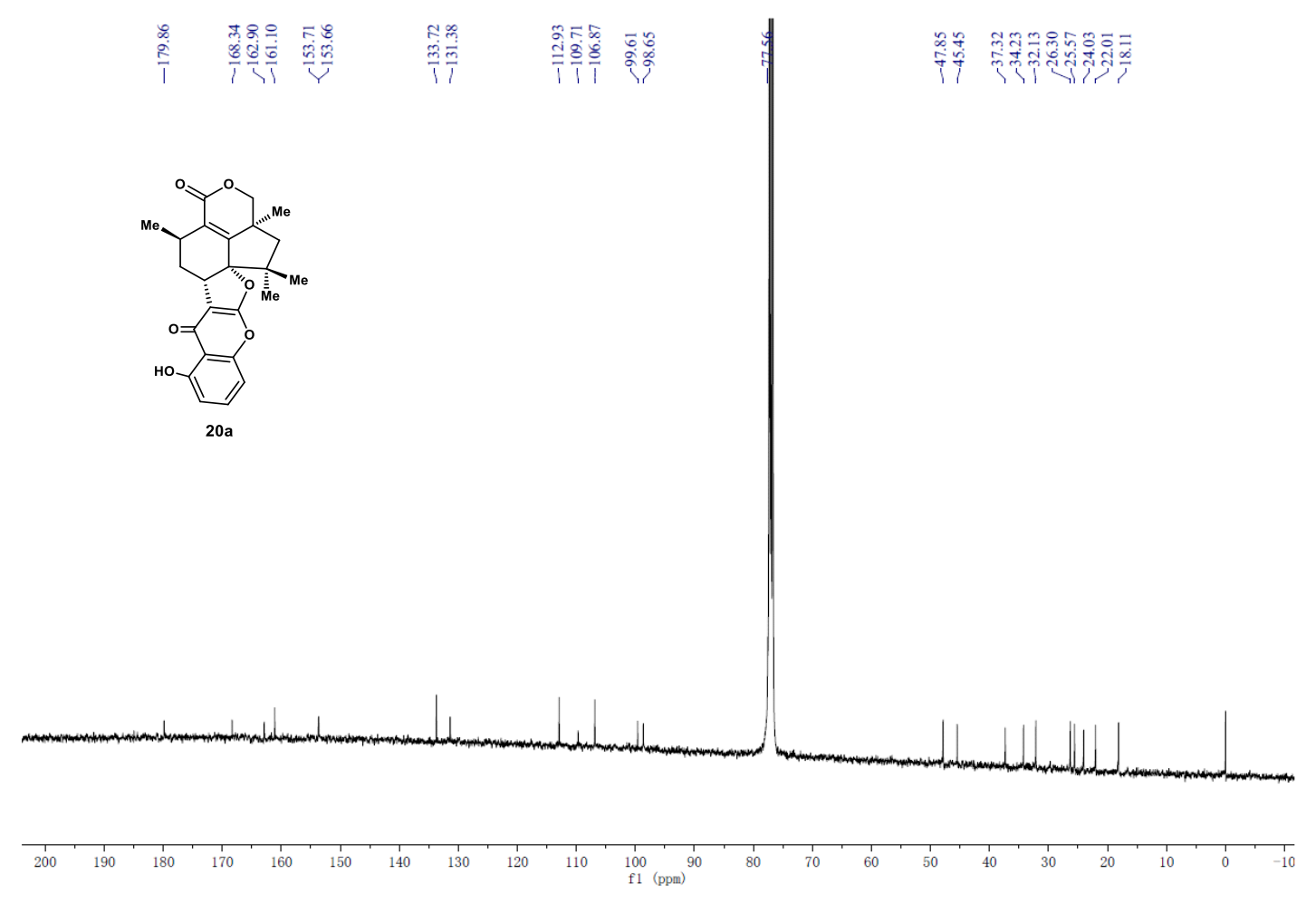

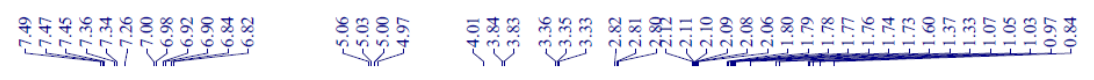
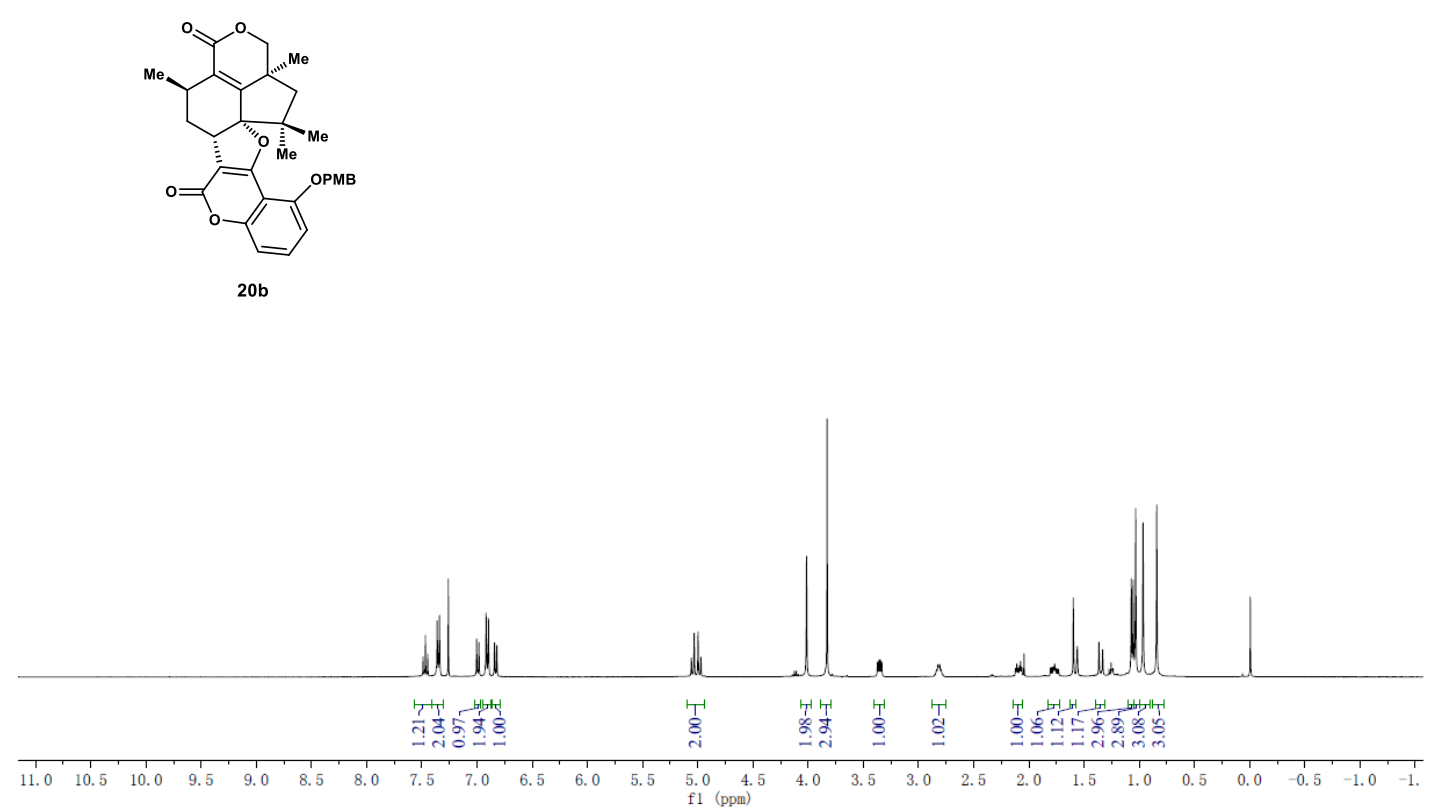


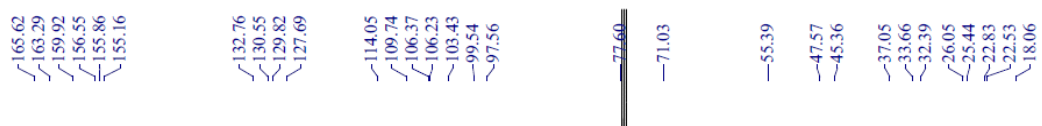

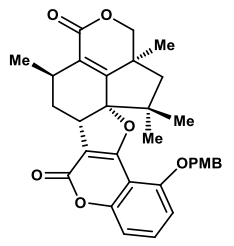

20b
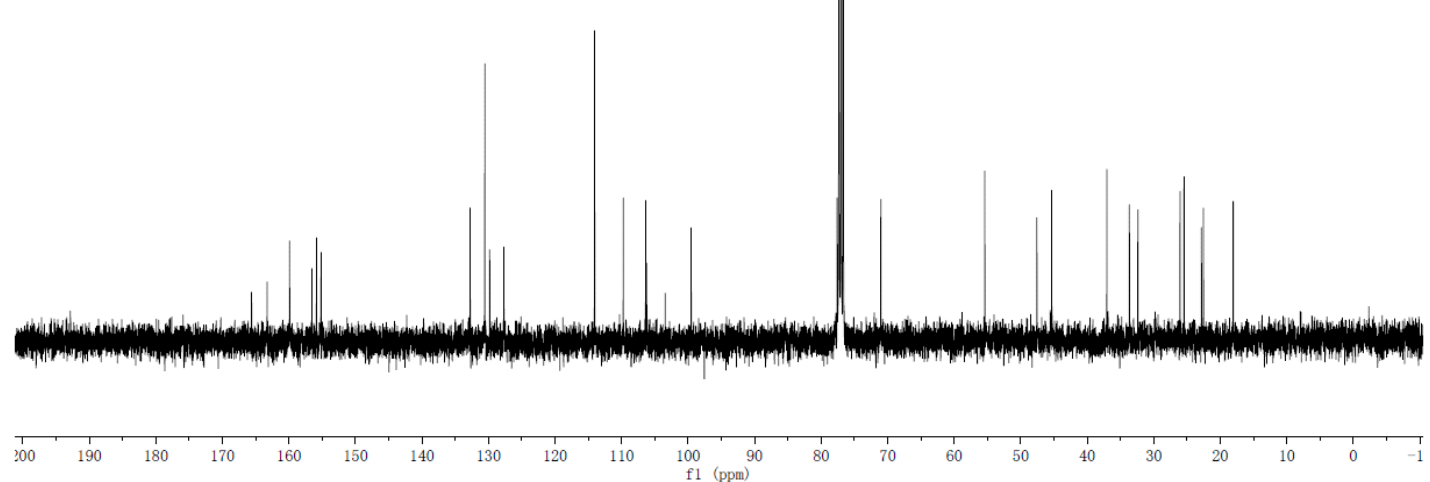

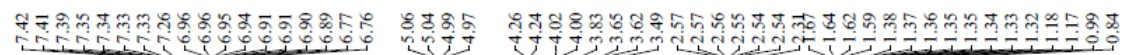

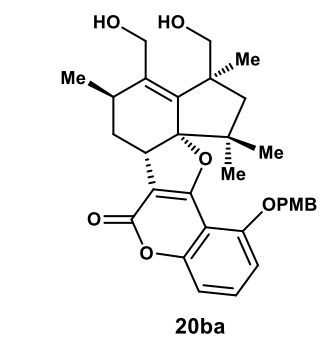

Ln. Mald

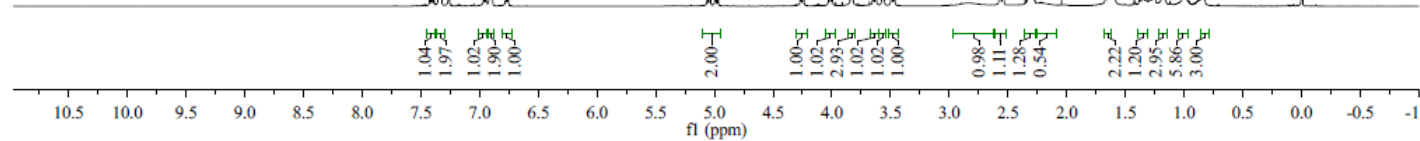



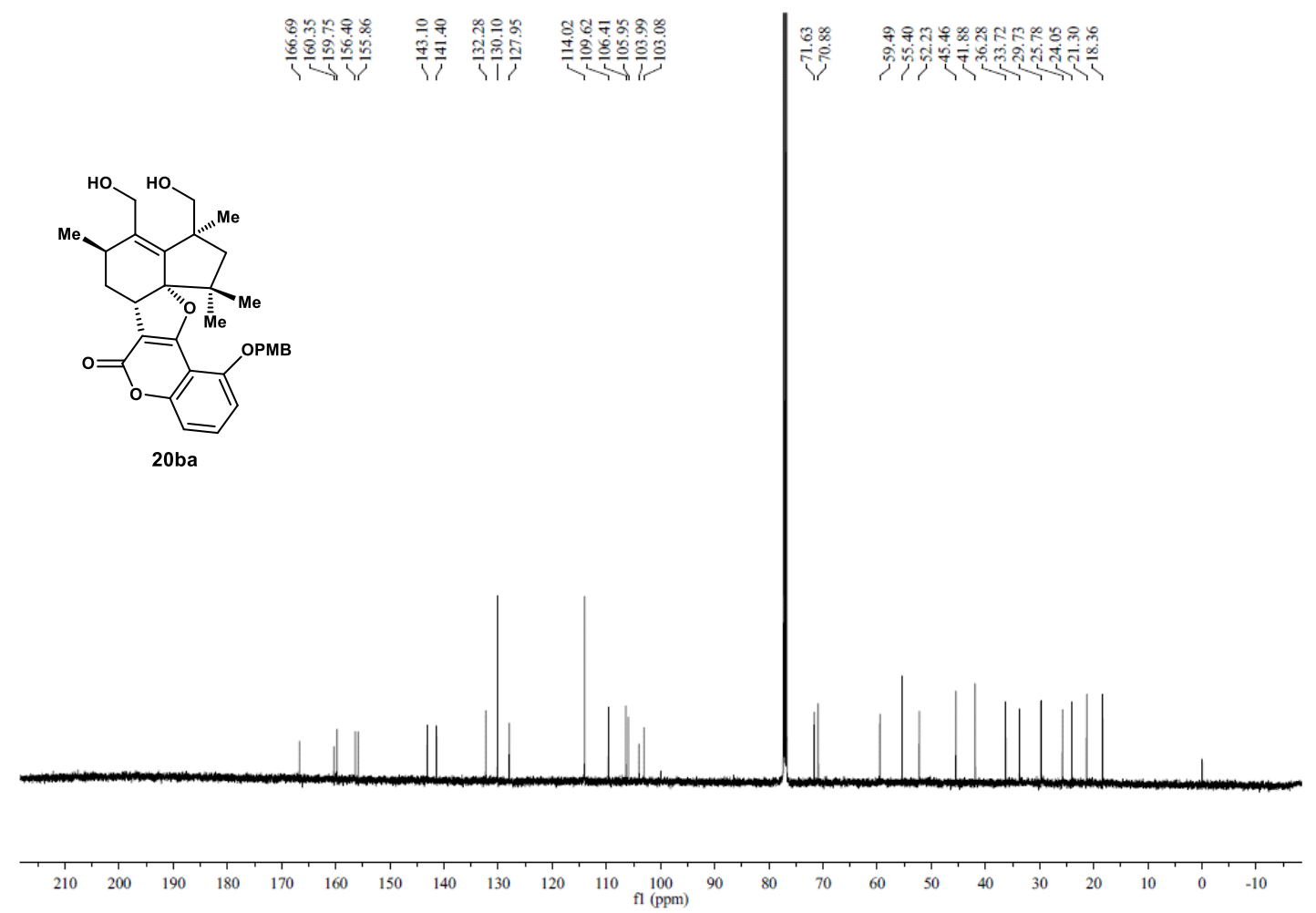

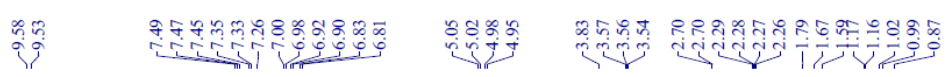
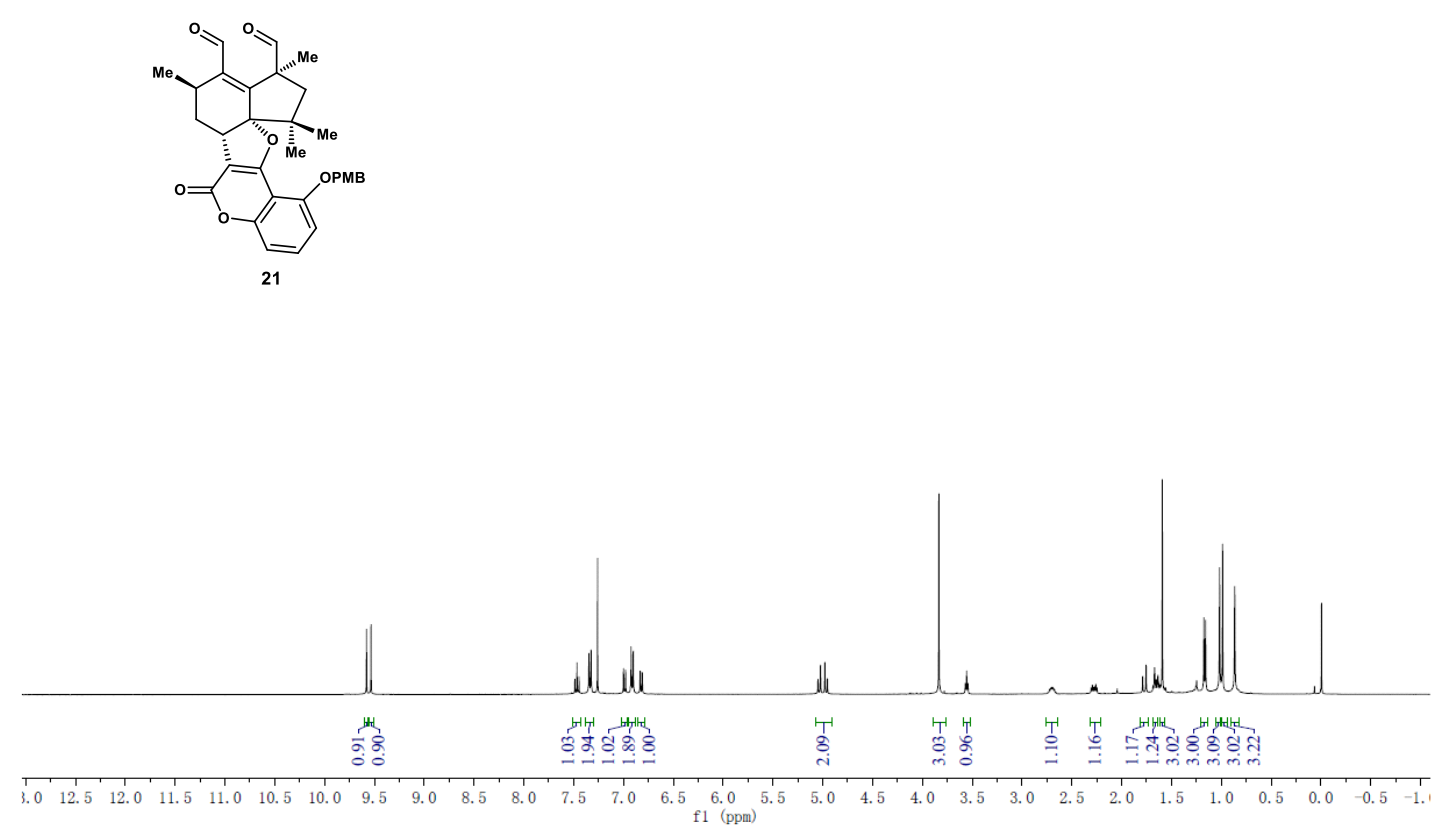


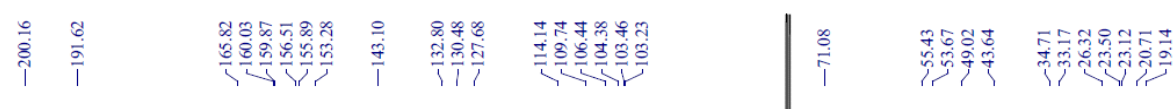
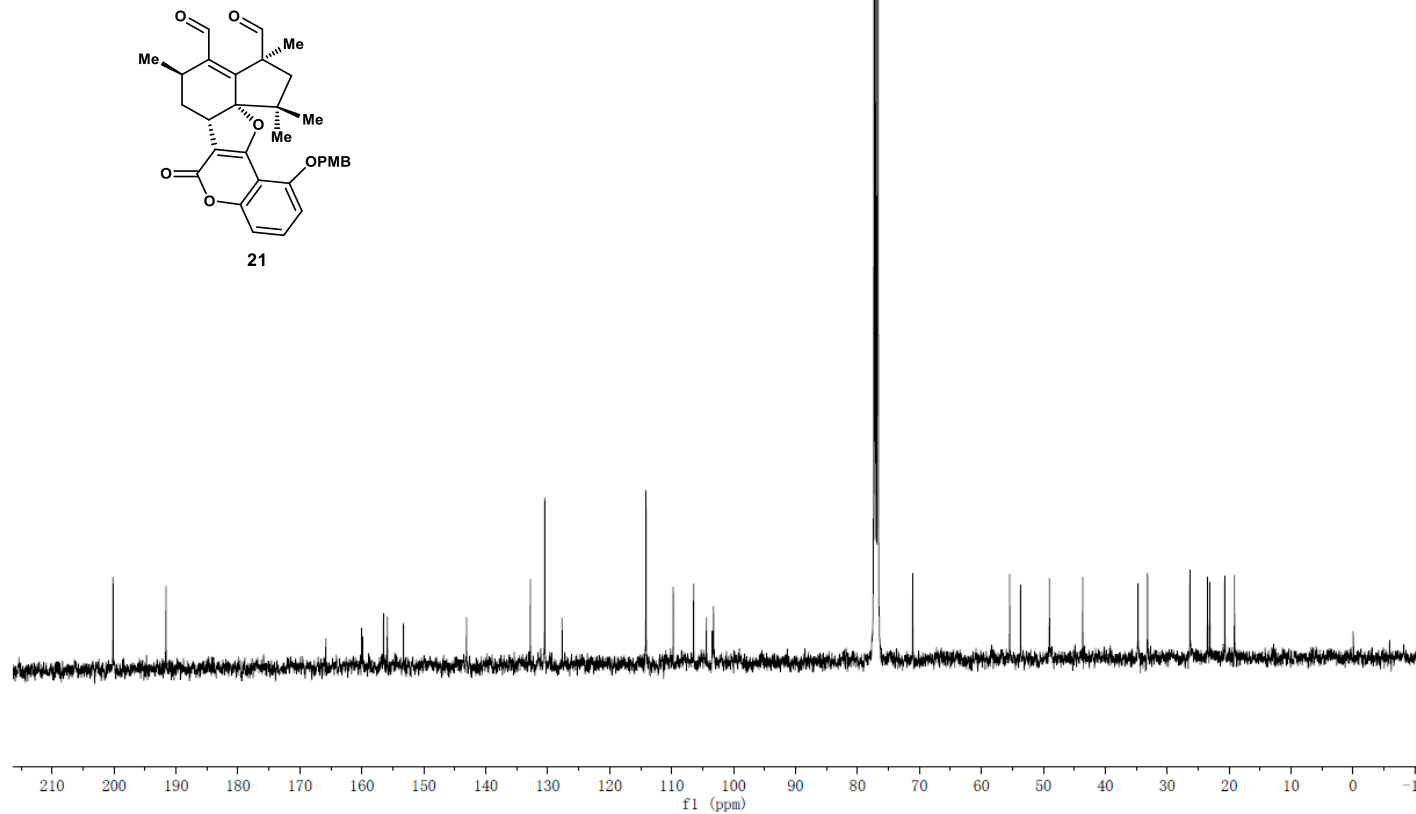

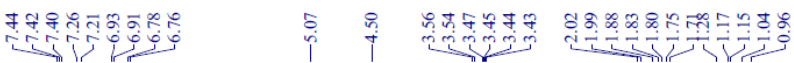

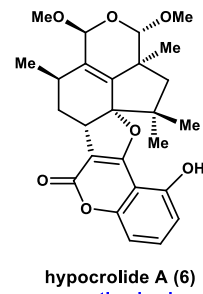

synthesized

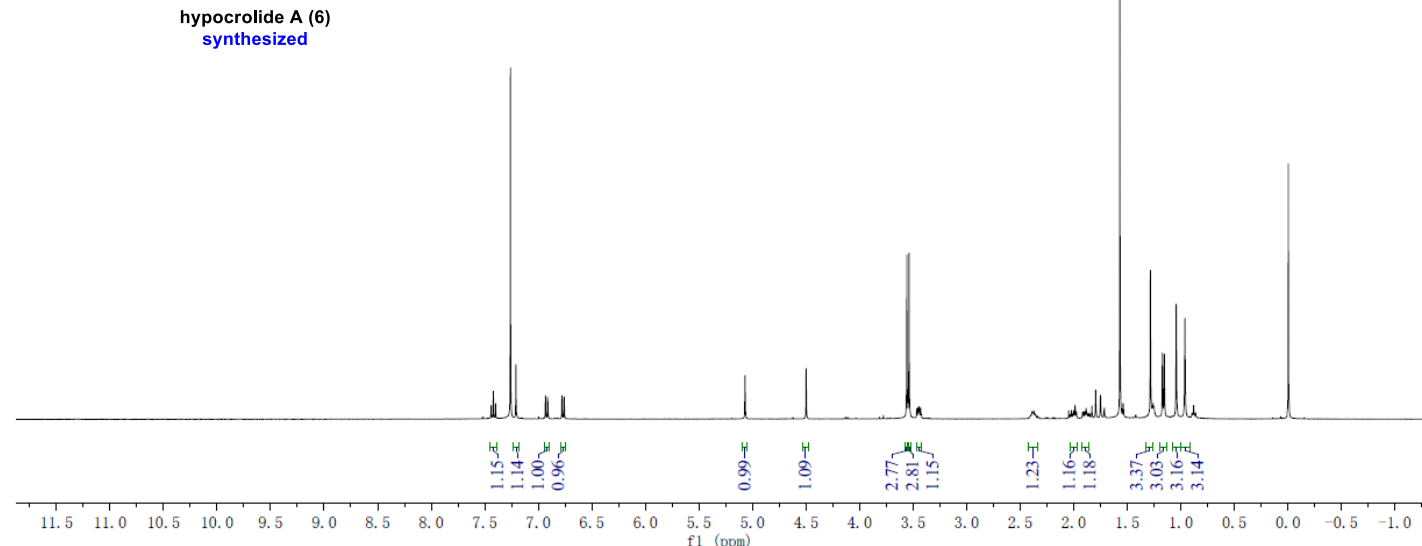



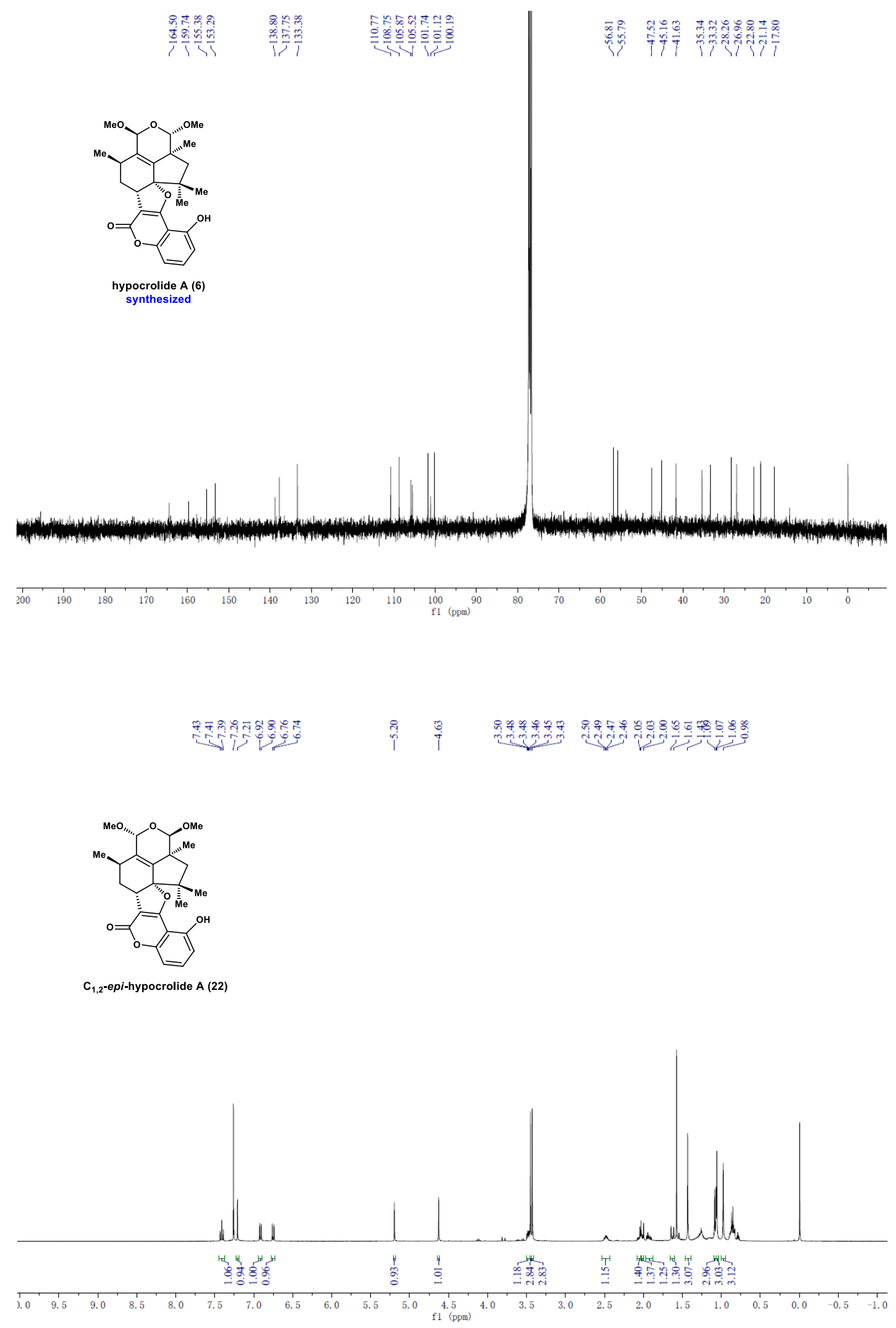


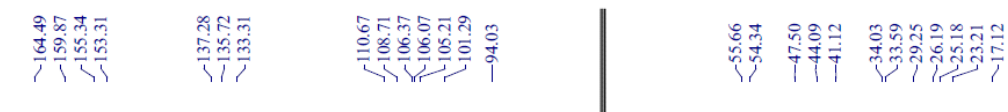

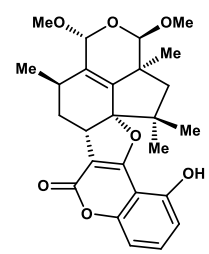

$\mathrm{C}_{1,2^{-}}$epi-hypocrolide A (22)
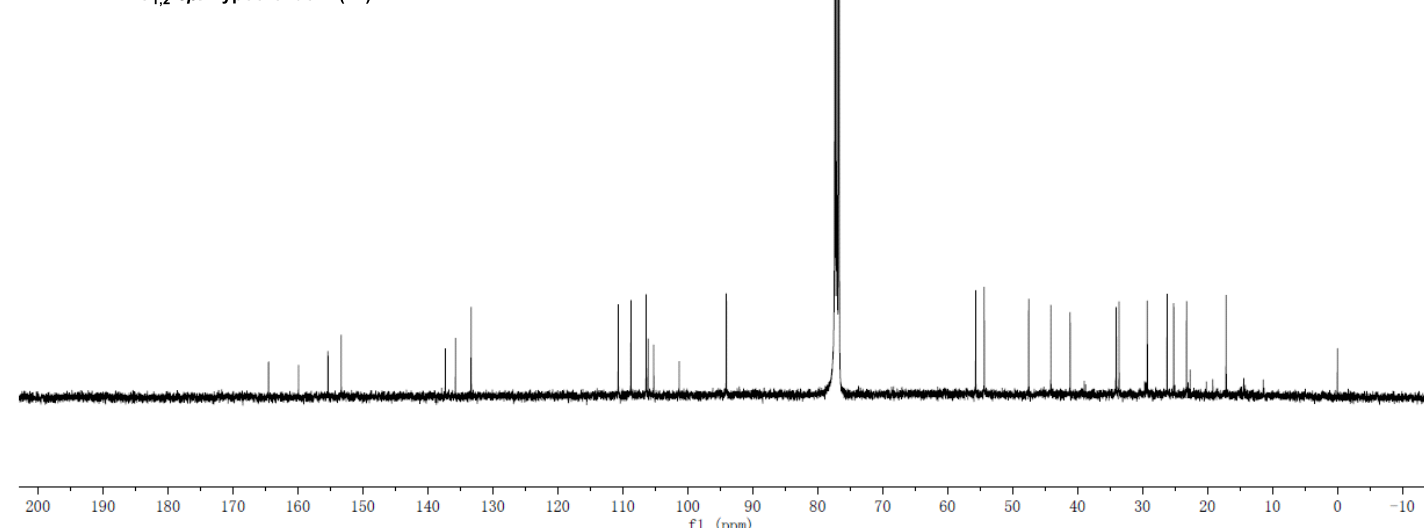

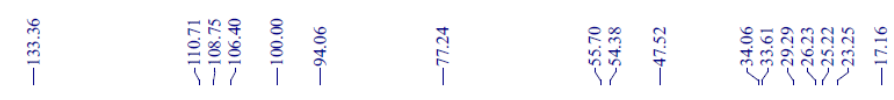

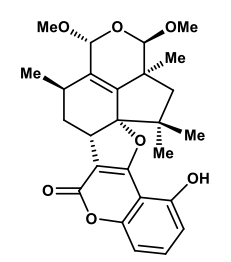

$\mathrm{C}_{1,2}$-epi-hypocrolide A (22) DEPT 135

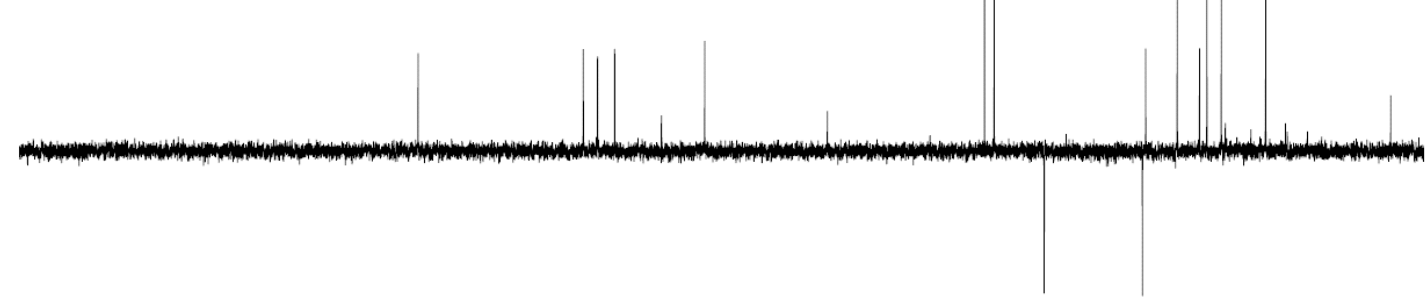

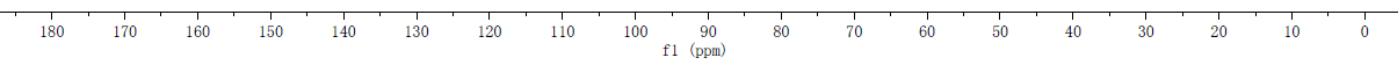



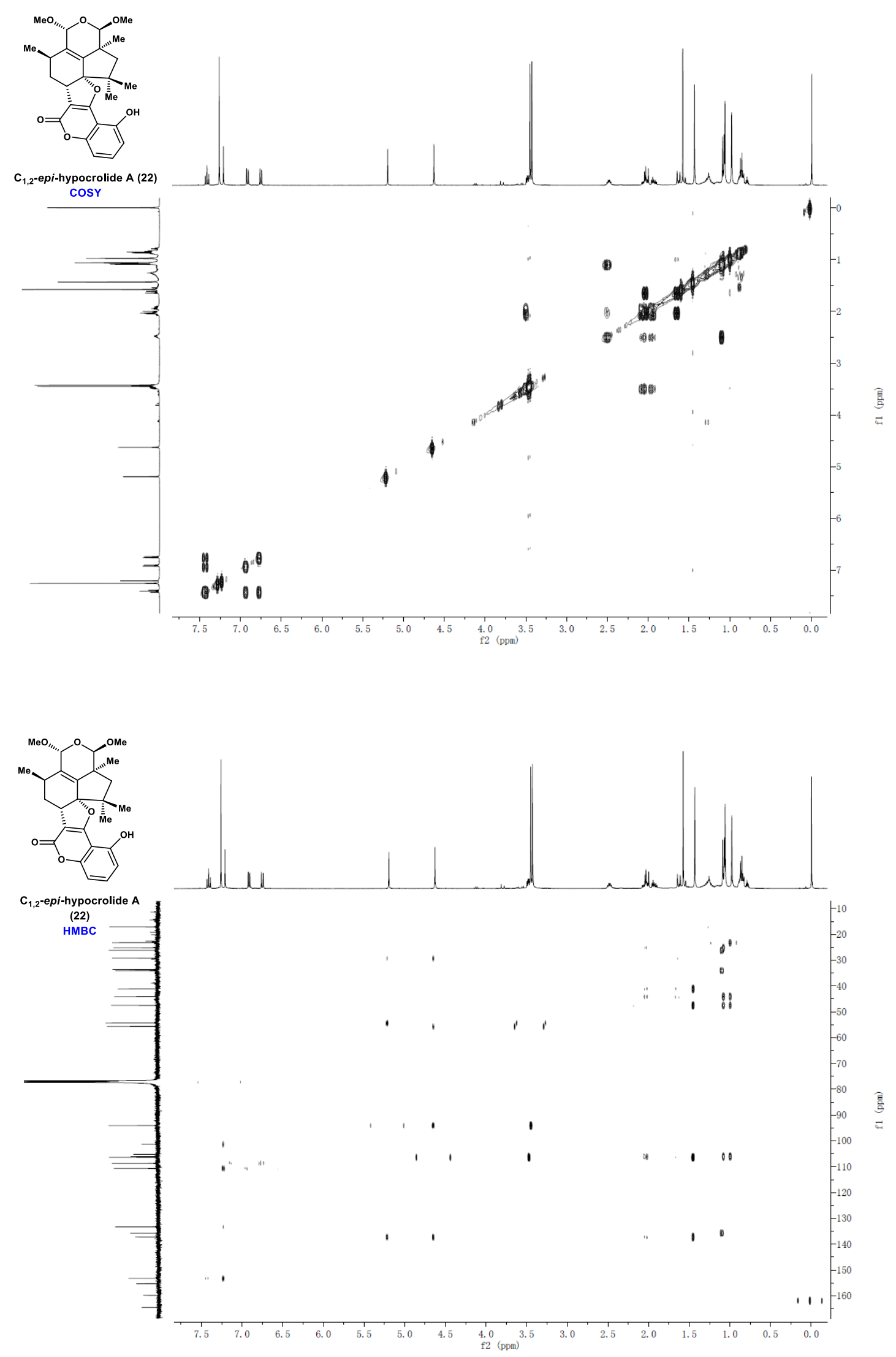

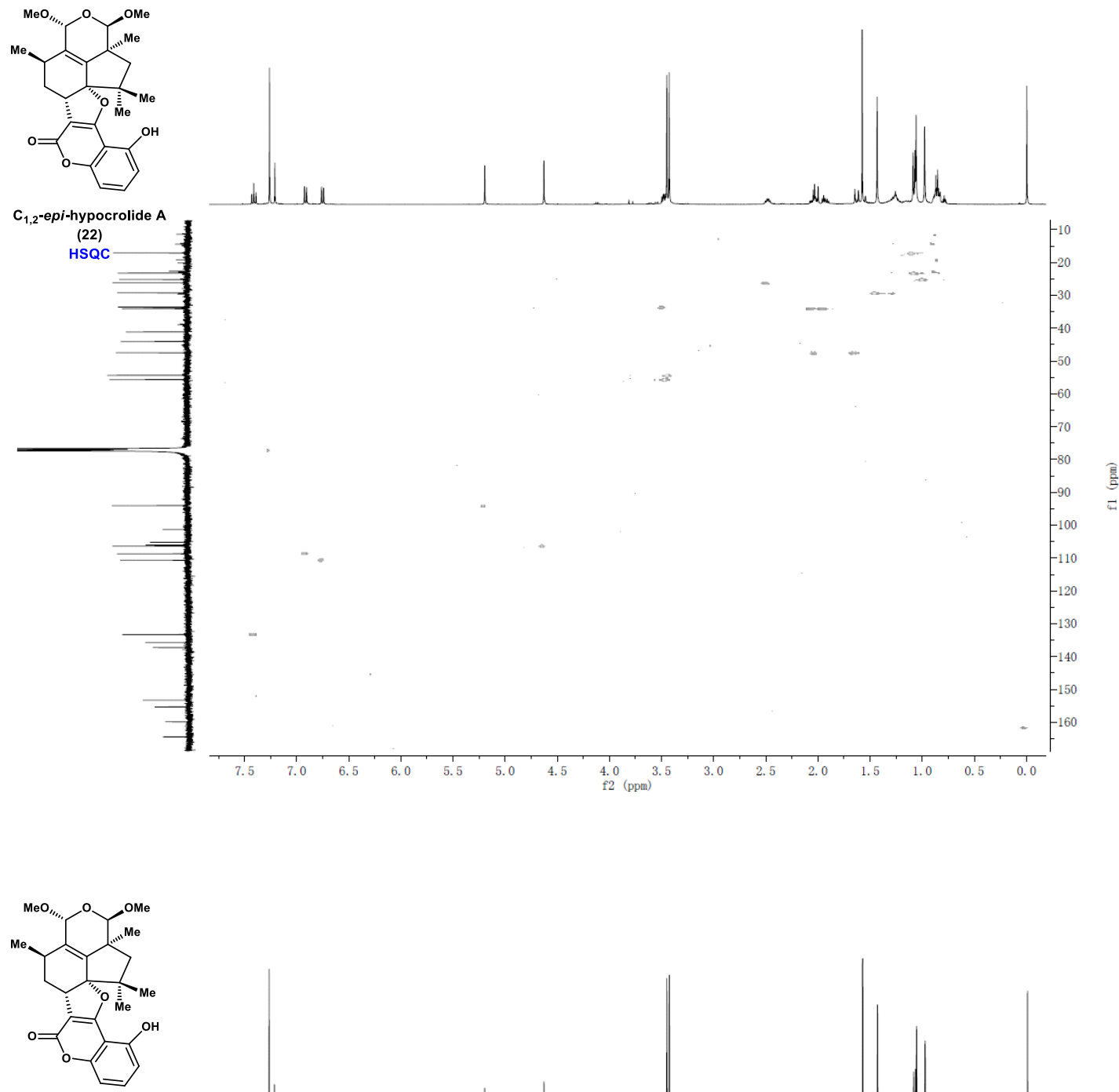

$\mathrm{C}_{1,2}$-epi-hypocrolide

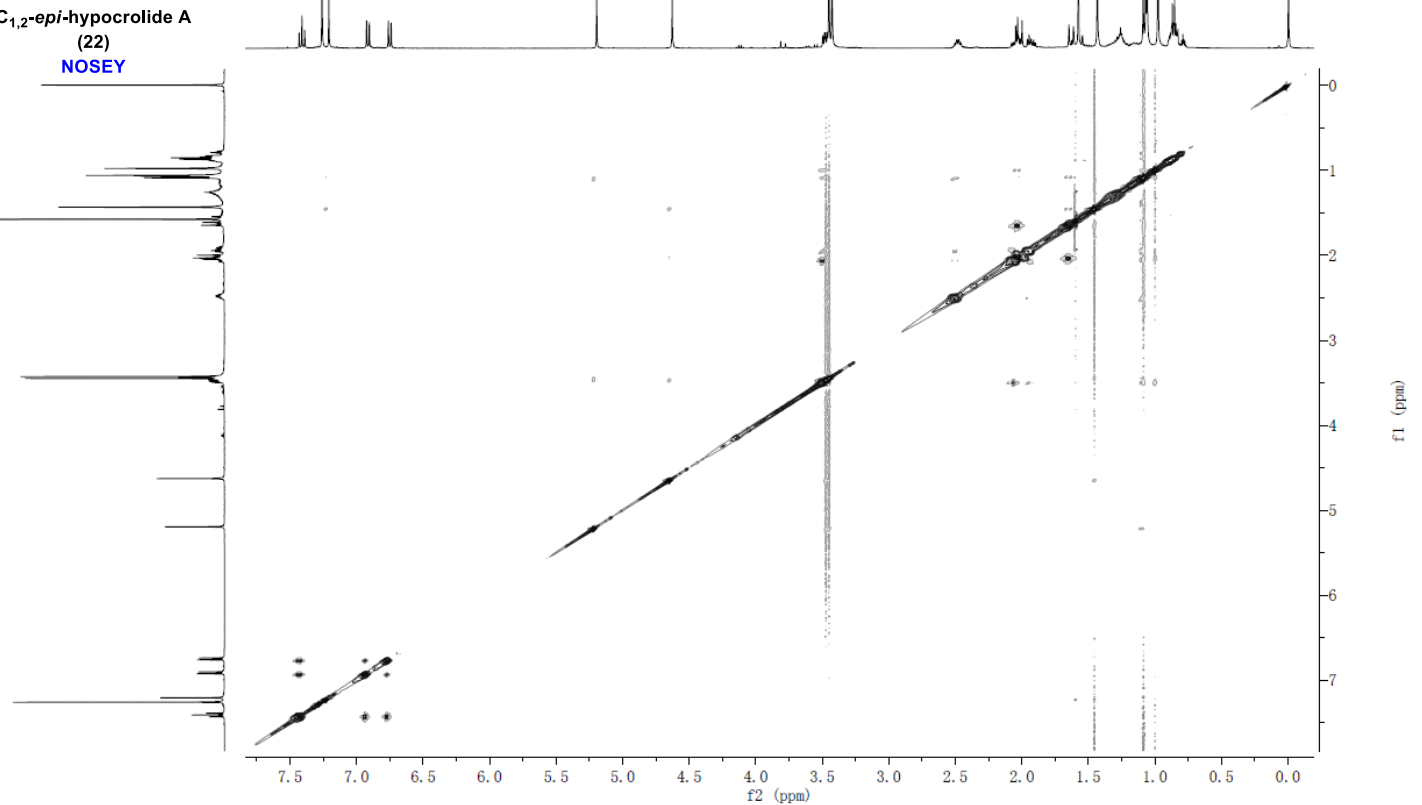



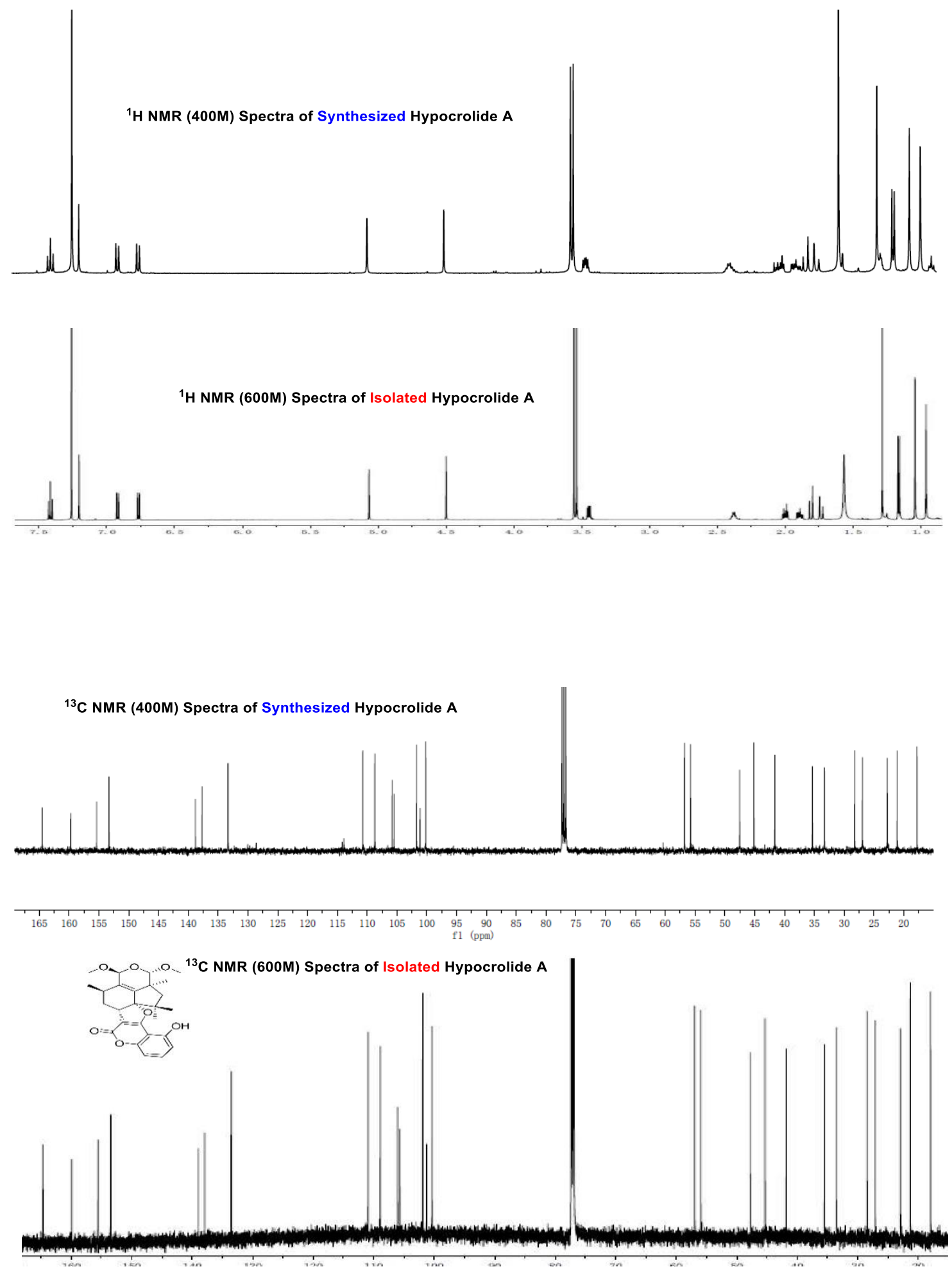

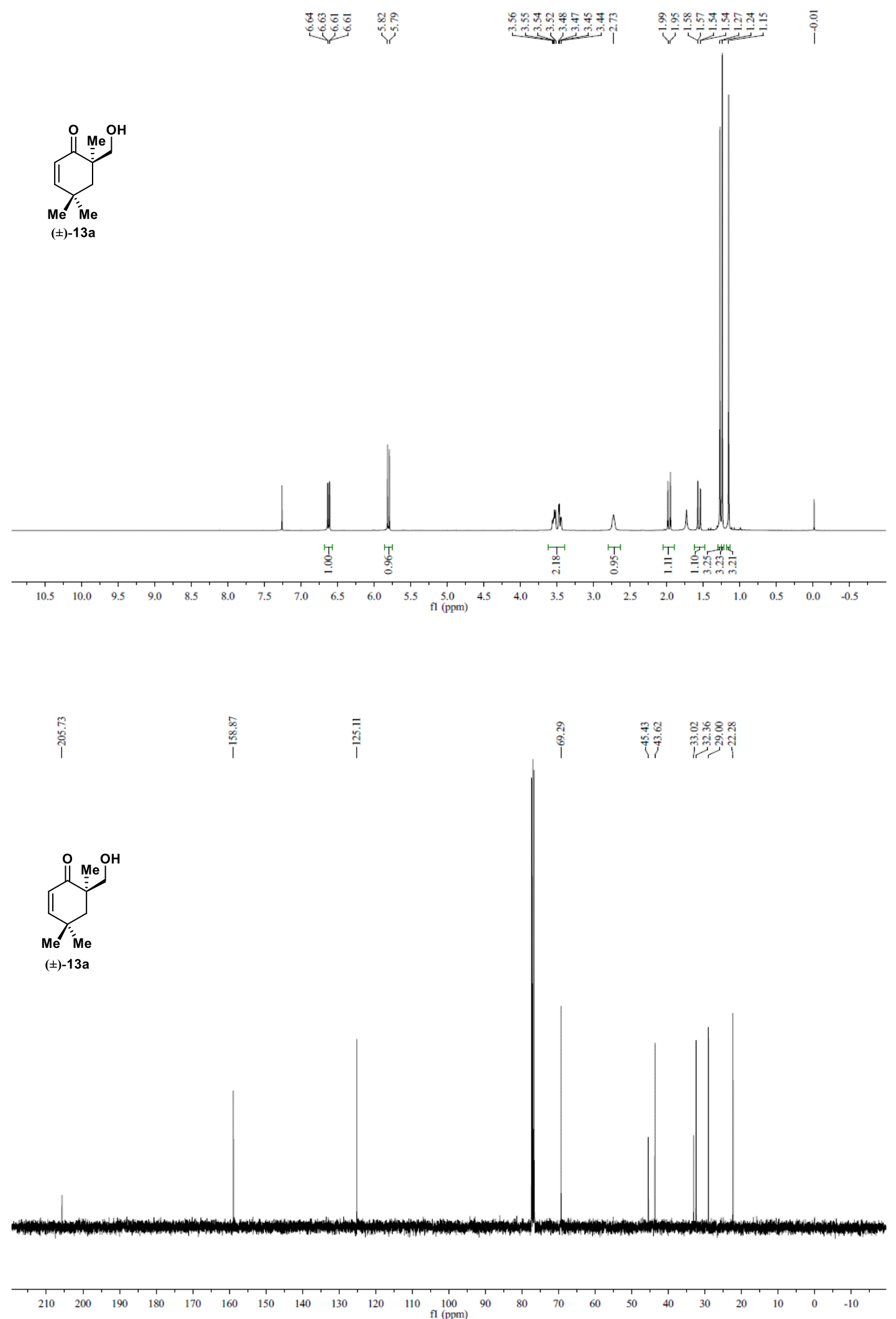


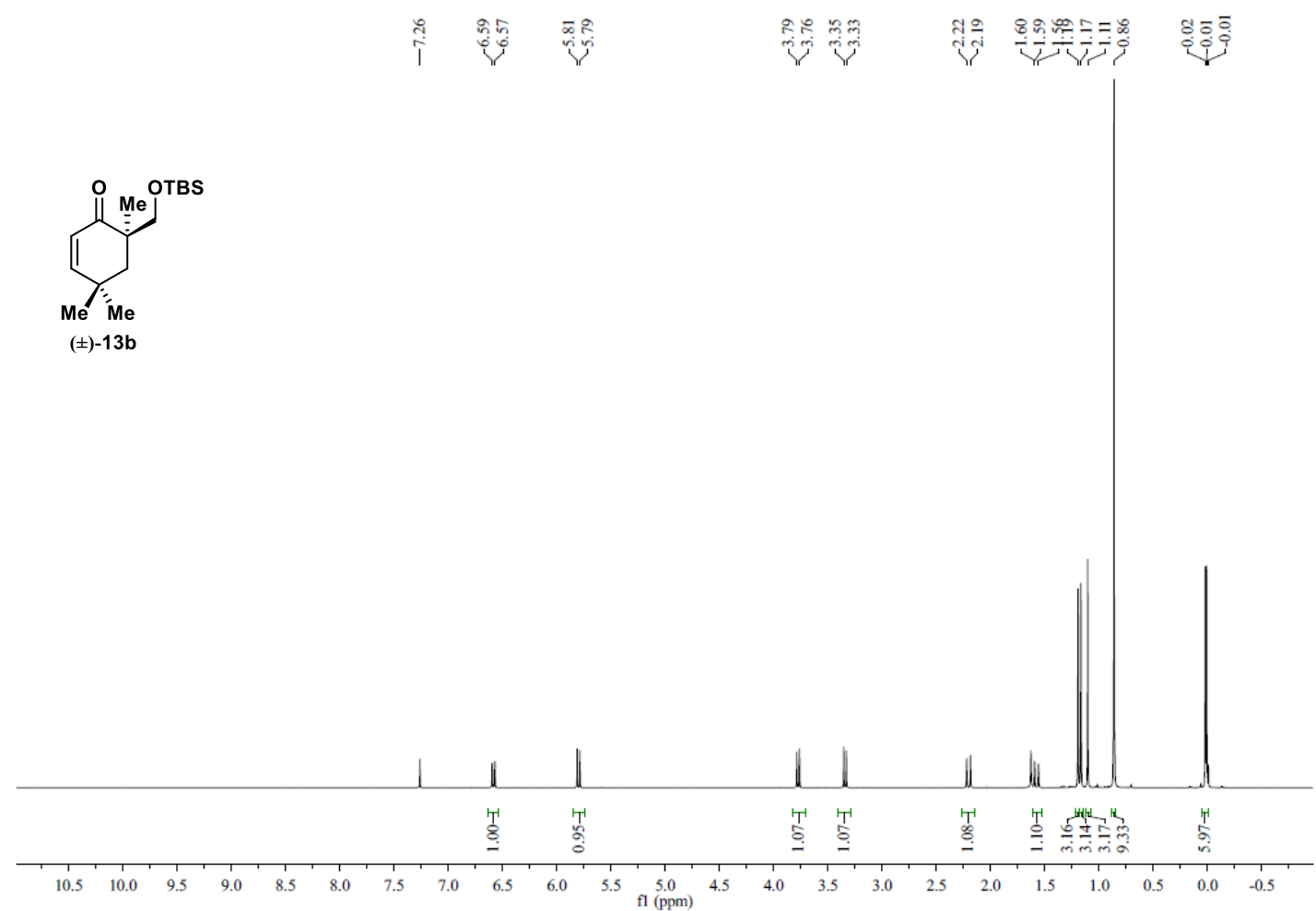

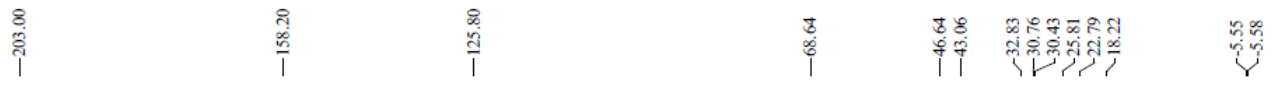

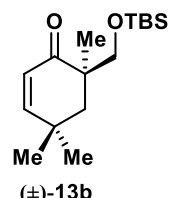

$( \pm)-13 b$

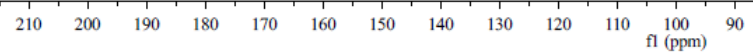

